\title{
INTERACTIONS OF TOLLMIEN-SCHLICHTING WAVES AND STATIONARY TRANSIENT DISTURBANCES
}

\author{
by \\ AKIIF MURAT GÜRÜN \\ Submitted in partial fulfillment of the requirements \\ For the degree of Doctor of Philosophy
}

Dissertation Adviser: Dr. Edward B. White

Department of Mechanical and Aerospace Engineering

CASE WESTERN RESERVE UNIVERSITY

January, 2006 


\section{CASE WESTERN RESERVE UNIVERSITY \\ SCHOOL OF GRADUATE STUDIES}

We hereby approve the dissertation of

\section{Akif Murat Gurun}

candidate for the Ph.D. degree *.

\begin{tabular}{l} 
(signed) $\frac{\text { Prof. Joseph M. Prahl }}{\text { (chair of the committee) }}$ \\
Prof. Iwan D. Alexander \\
\hline
\end{tabular}

Prof. Edward B. White

Prof. Robert V. Edwards

(date) $\underline{09 / 22 / 05}$

*We also certify that written approval has been obtained for any proprietary material contained therein. 
To my mother and brother, 


\section{Table of Contents}

Table of Contents

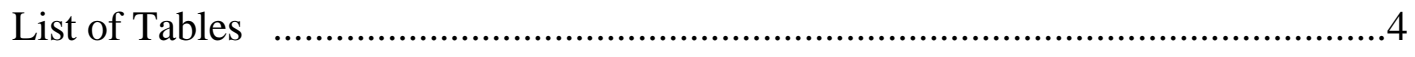

List of Figures

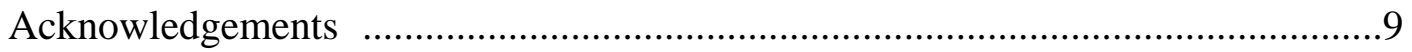

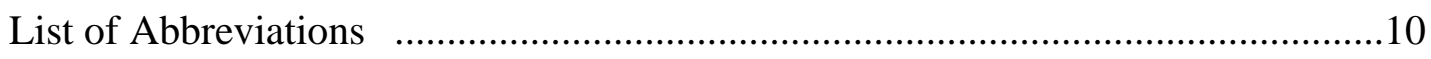

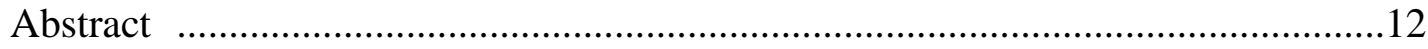

$\begin{array}{lll}1 & \text { INTRODUCTION } & 14\end{array}$

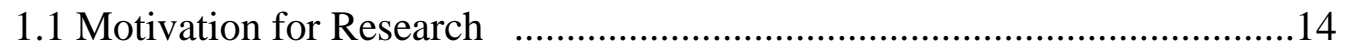

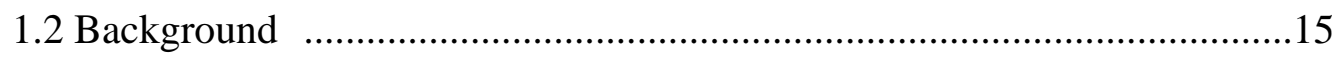

1.2.1 Transition Overview .........................................................15

1.2.2 Tollmien-Schlichting Waves ............................................18

1.2.3 Transient Growth ..............................................................20

1.2.4 Tollmien-Schlichting Waves Modulated by

Stationary Transient Disturbances ..............................................22

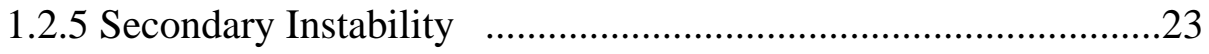

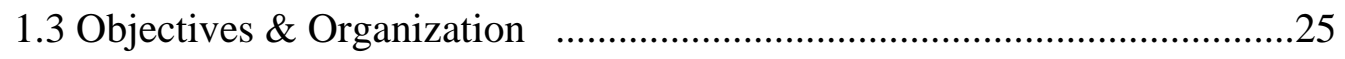




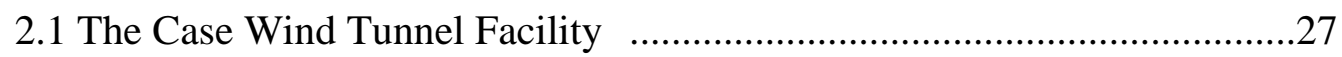

2.2 Flat Plate Alignment .............................................................................29

2.3 Disturbance Generation ................................................................................

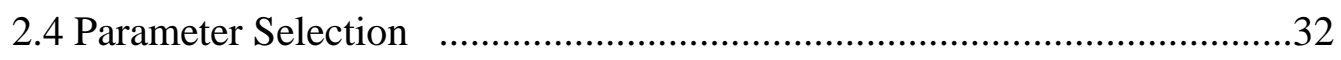

2.5 Experimental Procedure ............................................................................

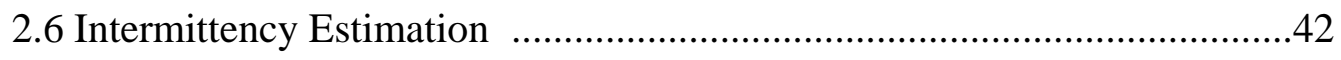

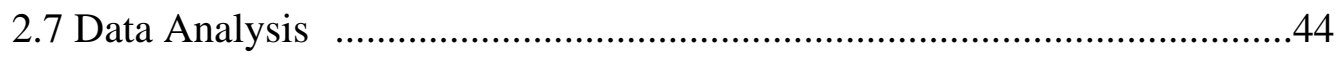

$3 \quad$ RESULTS \& DISCUSSION $\quad 47$

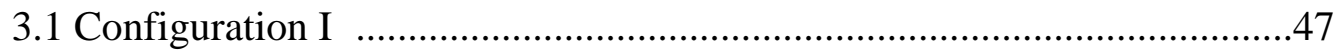

3.1.1 Baseline Case: TS waves in a spanwise-uniform

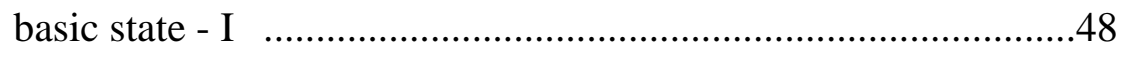

3.1.2 Spanwise Modulated Basic State - II ........................................50

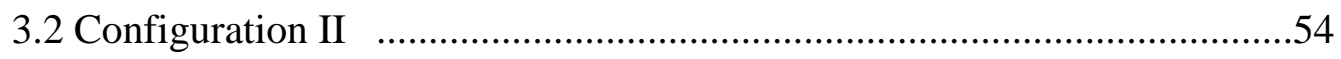

3.2.1 Baseline Case: TS waves in a spanwise-uniform

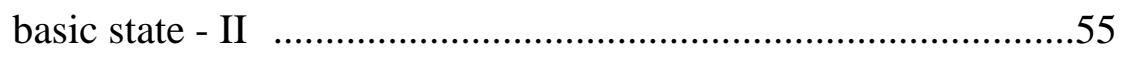

3.2.2 Spanwise Modulated Basic State - II ......................................57

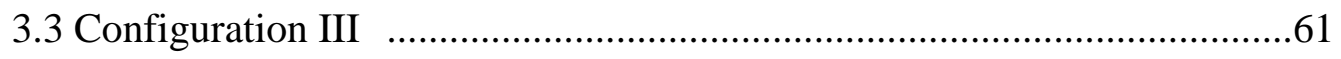

3.3.1 Spanwise Modulated Basic State - III ..........................................63 
4 SUMMARY AND CONCLUSIONS

$\begin{array}{lll}\text { A } & \text { FIGURES } & \mathbf{7 0}\end{array}$

BIBLIOGRAPHY

107 


\section{List of Tables}

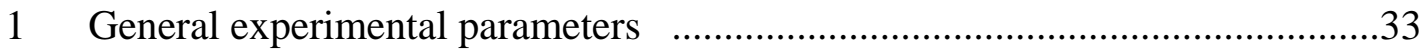

2 2D strip and 3D roughness element thicknesses, locations and thicknesses $\quad . .39$

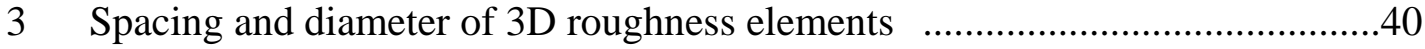

$4 \quad$ Breakdown locations and unsteady disturbance amplitudes at breakdown for all Configuration II cases ...................................................................60

5 Breakdown locations and unsteady disturbance amplitudes at breakdown for

Configuration III cases ..................................................................65 


\section{List of Figures}

1 Transition roadmap, adapted from Morkovin et al. (1994) ............................70

2 Wall normal Blasius mean flow and disturbance profiles of TS waves,

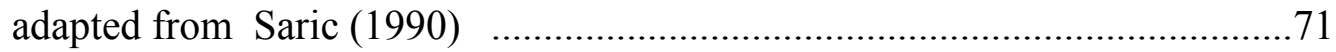

3 Schematic of TS amplitude change in the streamwise direction ....................71

4 Disturbance profiles computed by Cossu and Brandt (2002, 2004), Cases B to D

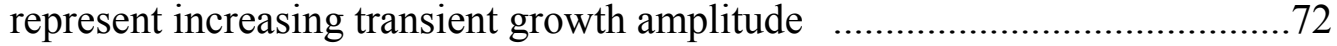

5 Development of spanwise peaks and valleys in the TS wave amplitudes, adapted

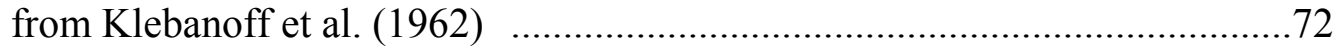

6 Patterns of $\Lambda$-type vortices: a) K-type b) C-type c) H-type,

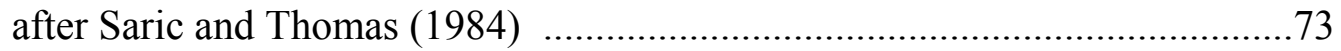

$7 \quad$ Sketch of boundary layer transition process, after White (1991) ...................73

8 (a) Sketch of Case wind tunnel. This sketch does not include the hotwire traverse system and only includes one turbulence screen

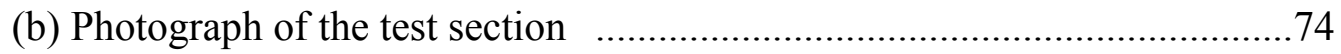

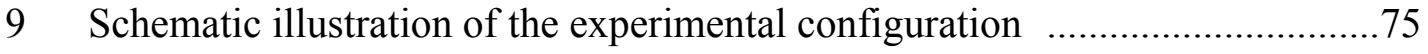

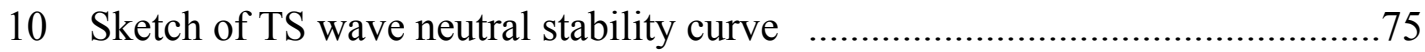

11 Typical total stationary disturbance energy variation,

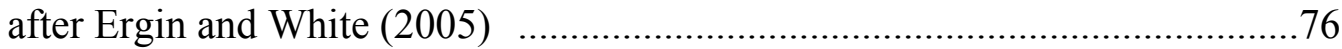


12 Intermittency Estimation Procedure

13 Conf. I - Displacement and Momentum Thicknesses, Shape Factor .80

14 Conf. I - Spanwise mean TS wave profile at $\mathrm{R}=845$

15 Conf. I - N-factor curve

16 Conf. I - $\mathrm{EB}_{\mathrm{rms}}$ and $\mathrm{A}_{\mathrm{s}}$ variation for $\mathrm{Re}_{\mathrm{k}}=153$

17 Conf. I - $\mathrm{E}_{\mathrm{rms}}$ and $\mathrm{A}_{\mathrm{s}}$ variation for $\mathrm{Re}_{\mathrm{k}}=238$

18 Conf. I - Displacement thickness variation and the velocity contours for $\mathrm{Re}_{\mathrm{k}}=153$ at $\mathrm{R}=814$ .84

19 Conf. I - Displacement thickness variation and the velocity contours for $\mathrm{Re}_{\mathrm{k}}=238$ at $\mathrm{R}=814$ .85

20 Conf. I - Evolution of the unsteady disturbance profiles in the high speed (left) and low speed streak (right) regions for $\mathrm{Re}_{\mathrm{k}}=153$, $\mathrm{R}=782,875$ and 946 (top to bottom) .86

21 Conf. I - Evolution of the unsteady disturbance profiles in the high speed (left) and low speed streak (right) regions for $\mathrm{Re}_{\mathrm{k}}=238$, $\mathrm{R}=782,875$ and 946 (top to bottom) 87

22 Conf. I - Spanwise modes of unsteady fluctuations for $\mathrm{Re}_{\mathrm{k}}=153$ (left) and $\mathrm{Re}_{\mathrm{k}}=238$ (right) at $\mathrm{R}=934$ .88

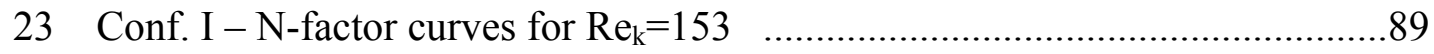

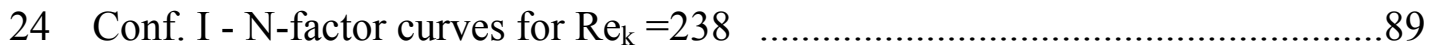

25 Conf. II - Displacement and Momentum Thicknesses, Shape Factor ...............90

26 Spanwise variation of TS wave amplitudes with no basic state modulation, $\mathrm{R}=860$ 


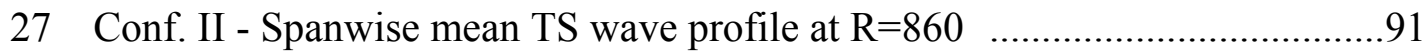

28 Spanwise averaged TS wave amplitude N-factors ....................................92

29 Streamwise development of intermittency with no basic state modulation .....92

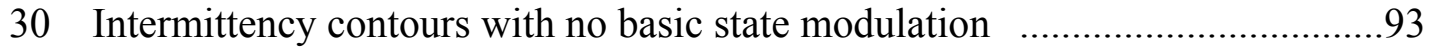

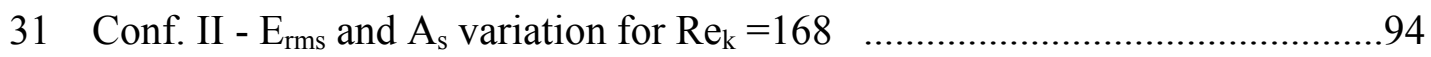

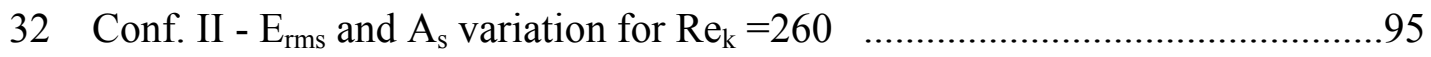

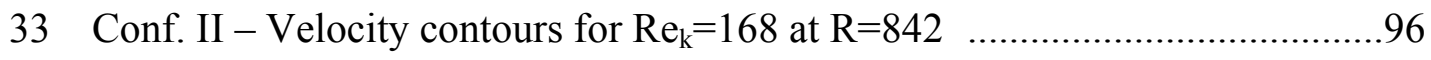

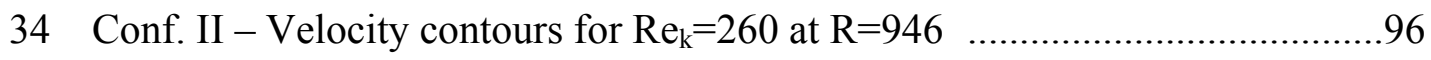

35 Conf. II - Evolution of the unsteady disturbance profiles in the high speed (left) and low speed streak (right) regions for $\mathrm{Re}_{\mathrm{k}}=168$, $\mathrm{R}=895,946$ and 1009 (top to bottom) .97

36 Conf. II - Evolution of the unsteady disturbance profiles in the high speed (left) and low speed streak (right) regions for $\mathrm{Re}_{\mathrm{k}}=260$, $\mathrm{R}=895,962$ and 1009 (top to bottom) .98

37 Conf. II - Spanwise modes of unsteady fluctuations for $\mathrm{Re}_{\mathrm{k}}=168$ (left) and $\mathrm{Re}_{\mathrm{k}}=260$ (right) at $\mathrm{R}=946$ .99

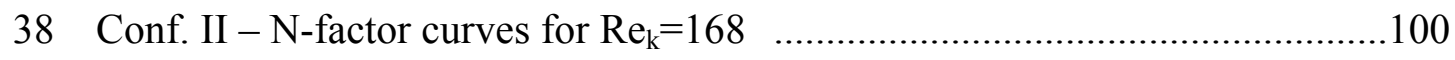

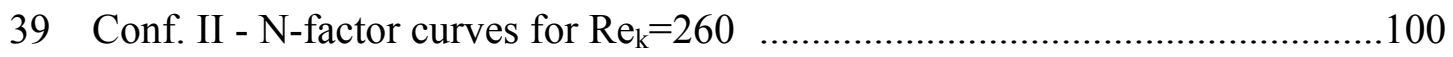

40 Contours of $10 \%$ Intermittency with modulated basic state (thin line) and amplitudematched TS waves for $\operatorname{Re}_{\mathrm{k}}=168$ .101

41 Contours of $10 \%$ Intermittency with modulated basic state (thin line) and amplitudematched TS waves for $\mathrm{Re}_{\mathrm{k}}=260$ . .101

42 Conf. III $-\mathrm{E}_{\mathrm{rms}}$ and $\mathrm{A}_{\mathrm{s}}$ variation for $\mathrm{Re}_{\mathrm{k}}=246$ 102 
43 Conf. III - Displacement thickness variation and the velocity contours for $\operatorname{Re}_{\mathrm{k}}=246$ at $\mathrm{R}=574$ 103

44 Conf. III - Evolution of the unsteady disturbance profiles in the high speed (left) and low speed streak (right) regions for $\mathrm{Re}_{\mathrm{k}}=246$, $\mathrm{R}=620,730$ and 759 (top to bottom) 104

45 Conf. III- Spanwise modes of unsteady fluctuations for $\mathrm{Re}_{\mathrm{k}}=246$ at $\mathrm{R}=663$ 105

46 Conf. III - N-factor curves for $\mathrm{Re}_{\mathrm{k}}=246$ .106

47 Conf. III - Intermittency variation across span for $\mathrm{Re}_{\mathrm{k}}=246$ .106 


\section{Acknowledgements}

I would like to thank my advisor, Prof. Edward B. White, for his continuous guidance and help. It was a privilege to have worked with him who is as good as a teacher as is a person.

Special thanks are extended to my committee members Prof. Prahl, Prof. Alexander and Prof. Edwards for their valuable suggestions and comments.

I would like to thank my fellow lab mates namely R. Balik, E. Birch, S. Bose, J. Kless, R. Kurtanich, M. Oliver, J. Rice, M. Shelley, and A. Song. They helped me with hundreds of hours of experimentation and also made this journey a lot more fun with their company. It was nice to know all of them.

I would like to express my deepest gratitude to my family for their endless support and love. Without them, I wouldn’t be within a lightyear of where I am now.

Finally, I would like to acknowledge the support of Case School of Engineering through Case Prime Fellowship.

This study was supported by the National Science Foundation under Grant number CTS-0245253. 


\section{List of Abbreviations}

A TS Amplitude

C.C. Complex conjugate

E Energy

$f \quad$ Dimensional frequency

F Nondimensional frequency, $F=2 \pi f v / U_{\infty}{ }^{2} \cdot 10^{6}$

H Shape Factor

$\mathrm{N} \quad$ Growth Factor, $N=\ln \left(A / A_{0}\right)$

PSD Power spectral density

R Boundary-layer-scale Reynolds number, $R=\sqrt{\mathrm{Re}}$

Re Reynolds number, $\operatorname{Re}=U L / v$

Re' Unit Reynolds number, $\operatorname{Re}^{\prime}=U_{\infty} / v$

$\operatorname{Re}_{k} \quad$ Roughness height based Reynolds number, $\operatorname{Re}_{k}=U(k) k / v$

U Streamwise mean velocity

$\mathrm{x}, \mathrm{y}, \mathrm{z} \quad$ Lab coordinate directions

\section{Greek Symbols}

$\alpha \quad$ Complex parameter, real part is the TS wave streamwise wavenumber Imaginary part is the associated spatial growth rate

$\beta \quad$ Dimensionless spanwise wavenumber, $\beta=\beta^{*} \delta$ 
$\beta^{*} \quad$ Spanwise wavenumber, $\beta^{*}=2 \pi / \lambda_{k}$

$\gamma \quad$ Turbulent intermittency

$\delta \quad$ Boundary layer dimension, $\delta=\sqrt{v x / U_{\infty}}$

$\delta^{*} \quad$ Displacement thickness, $\delta^{*}=\int_{0}^{\infty}(1-U) d y$

$\eta \quad$ Dimensionless wall normal coordinate, $\eta=y / \delta$

$\theta \quad$ Momentum thickness, $\theta=\int_{0}^{\infty} U(1-U) d y$

$\lambda_{k} \quad$ Roughness array spacing

$v \quad$ Kinematic viscosity

$\omega \quad$ Dimensionless frequency

\section{Subscripts}

$\begin{array}{ll}\text { i } & \text { Imaginary } \\ \text { r } & \text { Real } \\ \text { BL } & \text { Blasius } \\ \text { crit } & \text { Critical } \\ \text { k } & \text { Roughness height } \\ 0 & \text { Initial } \\ \text { rms } & \text { root-mean-square } \\ \text { vle } & \text { virtual leading edge } \\ \infty & \text { Freestream } \\ \text { I } & \text { Branch I } \\ \text { II } & \text { Branch II }\end{array}$


Interactions of Tollmien-Schlichting Waves and Stationary Transient Disturbances

Abstract

By

AKİF MURAT GÜRÜN

Numerical simulations have shown that stationary, spanwise periodic transient disturbances are capable of suppressing the growth of Tollmien-Schlichting (TS) waves in boundary layers. A natural implication is that the deliberate introduction of transient disturbances may delay TS-dominated transition. This experimental study investigates the interactions of these two types of disturbances and the breakdown of TS waves with and without transient disturbances. TS waves are created using a combination of acoustic forcing and a two-dimensional roughness strip while transient disturbances are created using a spanwise array of cylindrical roughness elements. It is found that in the presence of stationary transient disturbances, wall-normal velocity profiles of the unsteady TS-like waves are modulated and their growth rate is reduced. The modulated wall-normal velocity profiles have a characteristic M-shaped appearance and this shape appears to be mainly due to the component of the unsteady disturbances that varies on the spanwise wavelength of the stationary disturbances and higher spanwise harmonics of this wavelength. An important finding with respect to transition control applications is that the receptivity of the TS waves is reduced when the spanwise roughness array is present. 
The underlying mechanism responsible for this reduction is not yet known. Additionally, it has been found that unsteady disturbance amplitudes at breakdown are reduced when transient disturbances are introduced and, therefore, the breakdown location is actually moved upstream when care is taken to match initial unsteady disturbance amplitudes. It is concluded that even though TS growth is reduced, transition to turbulence is not successfully delayed. 


\section{Chapter 1}

\section{INTRODUCTION}

\subsection{Motivation for Research}

Understanding, predicting and controlling laminar-to-turbulent transition are of great interest to engineers because of the wide range of practical applications in which transition is significant. Generally, turbulent flow has an order of magnitude higher skin friction and heat transfer than an equivalent laminar boundary layer due to increased mixing between the boundary layer and freestream flow. The increased wall shear stresses due to turbulence result in significantly larger viscous drag on aircraft wings. It is estimated that fuel savings of up to $25 \%$ would be possible for a large commercial aircraft if laminar flow could be maintained over the wings (Thomas 1985, Saric 1994). On the other hand, separation and stall of low Reynolds number airfoils and turbine blades can be significantly improved if the boundary layer is turbulent. Additionally, better mixing properties of turbulence is an asset for designing efficient combustion systems and the increased heat transfer coefficient of turbulent flow is desirable in some heat exchanger designs. In gas turbine engines, an accurate estimation of the thermal loads on turbine blades strongly depends on the prediction of the onset of turbulence. The heat shielding requirement of hypersonic reentry vehicles, performance and detection of submarines and torpedoes are other applications for which predicting and controlling transition is essential. 


\subsection{Background}

\subsubsection{Transition Overview}

Fluid flows are generally referred to as being either laminar or turbulent. Laminar flow is ordered and layered while turbulent flow is chaotic and not predictable. Almost all laminar flows eventually develop (or transition) into turbulent flows. Even though the first studies in the field date back to the Nineteenth Century, transition to turbulence is still not fully understood and remains a puzzle to researchers today. The first transition experiment was conducted by Reynolds (1883). This consisted of studying the flow inside a glass pipe by introducing dye at the inlet. Reynolds observed that the dye remains along a nearly straight and distinct line if the flow is laminar but rapidly spreads across the pipe when the flow becomes turbulent. He found that a non-dimensional parameter, later called Reynolds number, $\mathrm{Re}=U D / v$, governs the onset of this transition. However, he also noted that there is no single critical Reynolds number at which the flow becomes unstable, it decreases as roughness and disturbances at the pipe entrance increase. On the other hand, no transition occurs below a certain Reynolds number regardless of the entrance's roughness.

The theoretical framework for understanding transition is based on the stability of small perturbations to a base flow using Navier-Stokes equations. First, Lord Rayleigh (1880) derived equations for the evolution of small disturbances for a parallel basic flow neglecting viscous and nonlinear terms. Rayleigh assumed a wave-like solution for the perturbation in both space and time of the form 


$$
u^{\prime}(x, y, z, t)=\hat{u}(y) e^{i(\alpha x+\beta z-\omega t)}
$$

and, using a Fourier transform, reduced the equation to an eigenvalue problem for exponentially growing or decaying disturbances. He showed that a necessary but not sufficient condition for inviscid stability is that the basic velocity profile has an inflection point. However, flows without inflection points like boundary layers with favorable pressure gradients are observed to be unstable at finite Reynolds numbers. Later, Prandtl (1921) showed that viscosity can be a destabilizing factor.

Following a procedure similar to Rayleigh's (1880), Orr (1907) and Sommerfeld (1908) included the viscous terms that resulted in the well-known Orr-Sommerfeld (OS) equation for the wall-normal disturbance velocity that describes the evolution of twodimensional disturbances. Squire (1933) derived a similar equation for the wall-normal disturbance vorticity to describe three-dimensional disturbances and proved that twodimensional (2D) disturbances grow faster than and become unstable upstream of threedimensional (3D) disturbances. This result is known as Squire's Theorem. This result led researchers to focus on 2D disturbances until recently. However, 3D disturbances are also found to cause transition through a mechanism called transient growth which will be explained in detail later in this chapter.

The first solutions of the OS equation for a Blasius boundary layer were obtained by Tollmien (1929) and these were later refined by Schlichting (1933). Tollmien and Schlichting analytically predicted the existence of two-dimensional unstable waves that are the normal mode solutions that grow or decay exponentially. For a zero-pressuregradient, flat plate boundary layer, these waves are referred to Tollmien-Schlichting (TS) waves. The existence of TS waves were experimentally verified by Schubauer and 
Skramstad (1947) once sufficiently low disturbance wind tunnels were developed that permitted the wave' detection.

Although the TS transition scenario is now well understood, transition to turbulence does not follow this path when the initial disturbances are large. Morkovin (1969) first coined the term "bypass" transition for cases in which known instability mechanisms (at the time, TS waves only) are bypassed. Transition triggered by large amplitude surface roughness and freestream turbulence are the prototypical bypass scenarios. Today, bypass transition generally refers to a transition whose disturbance growth mechanism is not described by the OS and Squire equations.

As a means of categorizing transition scenarios, Morkovin (1969) introduced a "transition roadmap". This roadmap was updated by Morkovin et al. (1994) and this updated roadmap is shown in Figure 1. The transition process can be categorized into three main stages: receptivity, disturbance growth and breakdown. Receptivity is the means by which disturbances enter the boundary layer and provide the initial conditions for disturbance growth. Disturbances like freestream turbulence, surface roughness and sound enter the boundary layer as steady and/or unsteady perturbations. This stage is not yet well understood in all situations but is critical because it provides the initial amplitudes, phases and frequencies of the disturbances.

Once disturbances enter the boundary layer, they proceed along one of the paths A-E depending not only on their initial amplitudes (as first put by Morkovin) but also, as recent thinking suggests, on other characteristics such as spatial wavenumbers and temporal frequencies. They grow or decay depending on the stability characteristics of 
the flow and multiple types of disturbances may coexist and possibly interact once they reach large amplitudes.

The final stage is breakdown to turbulence. In the case of TS waves (primary modes, path A) once the disturbances reach an amplitude near $1 \%$ of the freestream velocity, laminar flow can no longer be sustained and a more complex flow arises. This instability of the large-amplitude TS waves is referred to as a secondary instability because the primary disturbance is so large that it supports a second instability growth at frequencies that are not unstable in the original, unperturbed basic state. The secondary instability rapidly breaks down, turbulent bursts appear and these coalesce into fully developed turbulent flow. Detailed information about secondary instability is given later in this chapter. In the case of bypass transition, the disturbances break down without going through secondary instability and form turbulent bursts directly.

\subsubsection{Tollmien-Schlichting Waves}

Physically, TS waves are streamwise-traveling structures of spanwise-oriented vorticity. TS waves are the "primary mode" of transition for unsteady perturbations to a Blasius boundary layer basic state (path A in Figure 1). Mathematically, the disturbances take the form

$$
q^{\prime}(x, y, t)=\hat{q}(y) \mathrm{e}^{i(\alpha x+\beta z-\omega t)}+C . C .
$$

where $\hat{q}(y)$ is the mode shape, $\alpha$ is the streamwise wavenumber, $\beta$ is the spanwise wavenumber, $\omega$ is the frequency and C.C. stands for the complex conjugate. This form leads to linearized OS and Squire equations that can be solved for a given Reynolds 
number in two ways. First, $\alpha$ and $\beta$ can be assumed to be real and $\omega$ to be complex. In this case, a negative imaginary part of $\omega$ implies exponential growth in time; this is called temporal stability analysis. Alternatively, $\omega$ and $\beta$ can be assumed to be real and $\alpha$ to be complex. In this case, a positive imaginary part of $\alpha$ implies exponential growth in the streamwise direction; this is called spatial stability analysis.

The temporal analysis was the first to be performed because in the OS equation $\omega$ appears to the first power while $\alpha$ appears to the third power so the spatial problem is more difficult to solve. However, in open flows such as boundary layers, spatial stability analysis is more appropriate. Modern computing power makes the spatial problem straightforward and mode shapes and the growth rates can easily be obtained for any Reynolds number. Figure 2 shows the $|\hat{u}(y)|$ profile of a typical TS wave and the Blasius basic state $U(y)$ profile. In low-disturbance wind tunnels, it is possible for data to match these computed curves closely. Figure 3 is a schematic that shows the change of the TS amplitude in the streamwise direction at a given frequency.

The first point at which TS waves become unstable is called Branch I and is denoted by $R_{I}$ as shown in Figure 10. TS waves grow exponentially until Branch II, denoted by $R_{I I}$. Everywhere outside of this domain, the disturbances decay exponentially due to viscosity.

In an experiment or in flight, TS waves can be created via several mechanisms. The interaction of 2D freestream disturbances (acoustic waves) and a 2D roughness strip is one of the most common (Saric et al. 2002). The vibrations of the leading edge, vibrating ribbons and spanwise uniform suction and blowing are other effective methods. 


\subsubsection{Transient Growth}

For most of the last century, Squire's theorem prompted researchers to focus almost exclusively on 2D disturbances $(\beta=0)$. However, 3D disturbances such as those generated by $3 \mathrm{D}$ surface roughness or freestream turbulence are observed to be transitional upstream of $R_{I}$. Ellingsen and Palm (1975) identified a non-exponential growth mechanism called transient growth that operates below $R_{I}$ and provided the first explanation of this subcritical growth. Transient growth is an inviscid mechanism of algebraic energy growth and corresponds to path $\mathrm{C}$ in Figure 1. In a similar study, Hultgren and Gustavsson (1981) found that the temporal evolution of 3D disturbances in a boundary layer consists of a brief period of algebraic growth that is followed by exponential viscous decay.

A physical picture of transient growth is given by Landahl (1980) who described transient growth as a result of streamwise-oriented vortices that push the high momentum fluid from high in the boundary layer towards the wall and simultaneously push the low momentum fluid upwards in the boundary layer. This is called the lift-up effect. Lift-up creates spanwise modulations of the streamwise velocity called streamwise streaks. The streak growth is algebraic in time or space when a streamwise vortex exists but the streaks decay exponentially due to viscosity as the vortices lose strength.

One important difference between transient growth and TS waves is that the transient growth rate depends on the initial conditions set by the receptivity. TS wave growth at a certain frequency depends only on Reynolds number and is therefore insensitive to the receptivity mechanism. To address the fact that a range of growth rates 
are possible for transient disturbances, Farrell (1988) introduced the concept of optimal disturbances. The optimal disturbance is that which creates the largest possible transient growth over a certain length or time. Andersson et al. (1999), Luchini (2000) and Tumin and Reshotko (2001) have all addressed the spatial optimal disturbance problem. All found that optimal disturbances of the Blasius boundary layer consist of counter-rotating streamwise vortices resulting in alternating high and low speed streamwise streaks with a dimensionless spanwise wavenumber, $\beta=0.45$. Luchini found that for $\beta=0.45$, a stationary disturbance $(\omega=0)$ is optimal. Tumin and Reshotko also confirmed that $\alpha=0, \beta=0.45, \omega=0$ is optimal.

Mathematically, transient growth arises because the Navier-Stokes operator is non-orthogonal. This means that even where all OS and Squire modes are stable (exponentially decaying), the energy associated with the sum of the modes that describes a particular disturbance may briefly grow. Eventually, once the fastest decaying modes are no longer significant the transient disturbance decays at the rate of the most slowly decaying OS and Squire modes.

Because transient growth is most significant for $3 \mathrm{D}$ disturbances, it is receptive to surface roughness and freestream turbulence. Westin et al. (1994) investigated the effect of moderate levels of freestream turbulence (turbulence intensities around $1.5 \%$ of the freestream velocity) and observed that unsteady fluctuations grow algebraically with wall-normal profiles resembling Klebanoff modes. Klebanoff modes are very similar to the wall-normal profiles predicted by the optimal-disturbance theory and have been observed in numerous experiments on freestream turbulence, notably those by Klebanoff (1971) and Kendall (1985). Distributed surface roughness also induces transient growth 
through spanwise perturbations of the boundary layer. White and Reshotko (2002) verified that surface roughness is indeed a source of transient growth. White (2002) performed experiments with spanwise periodic surface roughness to compare data with the optimal disturbance theory. He found that even though the qualitative behavior is the same, streamwise vortices generate streamwise alternating streaks, discrepancies between the theory and measured data imply that roughness-induced disturbances are non-optimal. This finding does not imply that the concept of transient growth is incorrect but simply that optimal perturbations do not appear to be realizable in an experiment.

\subsubsection{Tollmien-Schlichting Waves Modulated by Stationary Transient Disturbances}

Studies of single instability mechanisms in isolation have produced good agreement between experiments and the theory. However, these studies rarely address the fact that, in general, multiple types of disturbances that grow via different mechanisms will co-exist and possibly interact with each other. A critical potential interaction is that between TS waves and transient disturbances, path B in Figure 1. Corke et al. (1986) examined the effect of distributed surface roughness on TS waves and observed that breakdown happens at lower amplitudes than in a baseline no-roughness case. Boiko et al. (1994) investigated the effect of freestream-turbulence-induced transient growth on TS waves and observed that there is a decrease in the growth rate of the TS waves but a considerable increase in the rate of turbulent spot production and this hastens transition relative to lower freestream turbulence values. 
Most recently, Cossu and Brandt $(2002,2004)$, hereafter referred to as CB, numerically predicted that moderate levels of optimal transient disturbances reduce the growth rate of TS wave and can lead to complete stabilization. These results suggest that inducing controlled transient growth might be used as a means of delaying transition. CB also found that transient growth leads to a spanwise modulation of the unsteady disturbance's wall-normal intensity profiles. This modulation results in M-shapes shown in Figure 4. In the calculations, the M-shapes are observed only in the spanwise invariant mode but not the fundamental and second spanwise modes.

\subsubsection{Secondary Instability}

Linear stability theory predicts the growth of small amplitude TS waves traveling in the streamwise direction. However, once the TS waves reach to certain amplitude, threedimensionality starts to develop and the TS waves cease to be spanwise uniform. This behavior was first deliberately explored by Klebanoff et al. (1962). They observed spanwise peaks and valleys of TS amplitudes and an associated system of streamwise vortices. They concluded that any shear layer in the unstable region has a strong capability of amplifying three-dimensional disturbances that must exist at some level in any experiment or practical situation. Figure 5 shows the development of spanwise variations in the streamwise fluctuating velocity as observed by Klebanoff et al (1962). They observed aligned vortices with spanwise locations fixed by spacers put under a vibrating ribbon. Later experiments showed that staggered vortices can also exist. Saric and Thomas (1984) performed smoke-wire visualization experiments that revealed both 
staggered and aligned $\Lambda$-patterns as shown in Figure 6. Herbert (1985) showed that threedimensional $\Lambda$-vortices are the result of a secondary instability that destabilizes once TS waves reach sufficiently high amplitudes. Herbert also proved that aligned $\Lambda$-vortices (the disturbances measured by Klebanoff et al. 1962) are formed by the fundamental mode of this secondary instability while the staggered $\Lambda$-vortices arise from the subharmonic mode. The fundamental, aligned $\Lambda$ vortices are called K-type vortices after Klebanoff. The subharmonic mode has two patterns which are named H-type after Herbert and C-type after Craik who first explained them as a superposition of a wave triad. Herbert (1988) reviews the types of the breakdown patterns.

K-type (fundamental mode breakdown) aligned $\Lambda$ vortices have the samewavelength as a TS wave and are observed for TS amplitudes of approximately $1 \%$ of the free-stream velocity, $U_{\infty}$. The spanwise length, $\lambda_{z}$ is about half of the streamwise wavelength, $\lambda_{x}$. C-type vortices appear at lower TS amplitudes $\left(0.3 \%\right.$ of $\left.\mathrm{U}_{\infty}\right)$ with staggered alignment (peak-following-valley) with $\lambda_{z} \approx 1.5 \lambda_{x}$. A second type of staggered alignment is H-type with $\lambda_{x} \approx 0.7 \lambda_{z}$ and appears around $0.6 \%$ of $U_{\infty}$.

The analytical and computational studies conclude that under the amplitude conditions where the fundamental K-type breakdown is experimentally observed, the subharmonic mode is the dominant mode. This was puzzling for some time. However, Singer et al. (1989) showed that in the presence of streamwise vorticity, the fundamental mode is preferred over the subharmonic mode. In wind tunnel experiments, streamwise vortices are almost always present due to turbulence screens and/or the inlet contraction and this results in the K-type breakdown to dominate. Transient growth and the surface roughness that creates it are also sources of streamwise vortices so it is reasonable to 
expect K-type breakdown as part of a Path B interaction between transient disturbances and TS waves.

It is useful to sum up the transition process at this point. Figure 7 (White 1991) shows an overall picture for TS waves-dominated breakdown. The flow starts as a stable laminar one but 2D TS waves form once the critical Reynolds number is exceeded. These unsteady waves grow in amplitude and the modified flow becomes susceptible to 3D secondary disturbances that arise from sources such as those provided by transient growth. Peaks and valleys start to appear in the fluctuating velocity and breakdown following one of the $\Lambda$-type patterns (K-type, H-type or C-type) occurs. The turbulent spots eventually coalesce and form fully-developed turbulent flow.

\subsection{Objectives and Organization}

The present study aims to address the growth and breakdown of TS waves in the presence of stationary transient disturbances. This is the first deliberate study to document this interaction across the stages of receptivity, growth and breakdown. The first objective is to determine experimentally whether the growth rate reduction and the M-shape modulations of TS waves can be observed in the presence of sub-optimal transient disturbances as numerically predicted by $\mathrm{CB}$ for optimal disturbances. This is achieved by generating controlled TS waves using acoustic forcing and 2D roughness while also generating controlled stationary transient disturbances using a spanwise array of 3D surface roughness elements. Details about the disturbance generation will be given in Chapter 2. The second objective is to examine TS wave breakdown to turbulence in the 
presence of transient disturbances by measuring the distribution of turbulent intermittency across the experimental domain. The amplitudes of TS waves at breakdown are compared when transient disturbances are and are not present. Even though the growth rate may be reduced, the flow may be more prone to be turbulent if breakdown amplitudes are observed to be lower when transient disturbances are introduced.

This dissertation is organized as follows. Chapter 1 introduces the concepts of transient growth, TS waves and breakdown to turbulence. Chapter 2 describes the experimental facility, several experimental setups and the approaches to data analysis. Chapter 3 presents the results grouped into three configurations each of which gives additional insight into the objectives. Chapter 4 summarizes the results and concludes. 


\section{Chapter 2}

\section{EXPERIMENTAL SETUP AND APPROACH}

\subsection{The Case Wind Tunnel Facility}

All of the experiments of the present work are performed in the Case Western Reserve University Wind Tunnel. The facility is an open-loop, subsonic tunnel with a $710 \mathrm{~mm} \times$ $710 \mathrm{~mm} \times 2.7 \mathrm{~m}$ test section and a maximum operating speed of $25 \mathrm{~m} / \mathrm{s}$. A sketch of the wind tunnel and a photograph with additional details of the test section is given Fig. 8a and $8 \mathrm{~b}$ respectively. The flow is treated using a variety of techniques to achieve low levels of freestream turbulence which is essential for boundary layer stability experiments. Air enters the wind tunnel through a contoured inlet fairing and then passes through a $100-\mathrm{mm}$-thick thick honeycomb flow straightener with $8 \mathrm{~mm}$ cells. The flow then passes through four high-tension, 65\% open area, stainless steel screens that break large turbulent eddies into faster-decaying smaller sizes and improve the mean flow quality. Next, the flow passes trough a 6.25:1 fifth-degree polynomial-shaped contraction cone that further enhances the turbulent dissipation. Every section of the tunnel is independently isolated on pneumatic mounts and is connected to each other only by flexible tape. This prevents vibrations from the motor and other sources from propagating into the test section. By the time flow reaches test section, the freestream turbulence intensity is only approximately $0.05 \% U_{\infty}$ at $12 \mathrm{~m} / \mathrm{s}$ White (2002). Low frequency acoustic fluctuations amount to about $0.4 \% U_{\infty}$ at the same speed. 
The test section contains a flat plate mounted vertically $250 \mathrm{~mm}$ from the interior of the front wall. The plate is $1100 \mathrm{~mm}$ long, $635 \mathrm{~mm}$ wide, $9.5 \mathrm{~mm}$ thick and has a 25 $\mathrm{mm}$ elliptical leading edge. The plate is aluminum and hand polished to a near mirror finish. It is attached to the top and bottom walls of the tunnel by four-adjustable brackets that enable proper alignment of the tunnel to provide zero-pressure-gradient Blasius boundary layer. Additionally, an adjustable $300 \mathrm{~mm}$ trailing-edge flap ensures that the stagnation point is on the test side of the flat plate.

The test section is also equipped with a three-degree-of-freedom traverse that can move hotwires and other instruments $1700 \mathrm{~mm}$ in the streamwise direction $(x), 100 \mathrm{~mm}$ in the wall-normal direction $(y)$ and $172 \mathrm{~mm}$ in the spanwise direction $(z)$. The spatial resolution is $6.4 \mu \mathrm{m}$ in $x$ and $3.2 \mu \mathrm{m}$ in $y$ and $z$.The traverse's sting supports two hotwire probes that measure air velocity. One probe is located in the boundary layer and the other is located in the free-stream. Positioning is controlled by a Velmex 9000 controller that communicates with the main control computer via a RS-232 line.

The air flow is powered by a fan driven by a belt and pulley system connected to a 20 HP DC electric motor. The motor is controlled by the main control computer via analog DC signals and a Reliance Electric FlexPak 3000 controller.

The velocity measurements are performed by a constant temperature anemometer system, an A.A. Labs model AN-1003. The system can accommodate up to six hotwire channels; only two are required by this study. Boundary layer type tungsten hotwires manufactured by Dantec that are $1 \mathrm{~mm}$ in length and 2.5 or $5 \mu \mathrm{m}$ in diameter are used as sensors. The wires' overheat ratio is 1.8 . 
Data are acquired using a PCI-MIO-16XE-10 model 16 bit resolution card with a maximum sampling rate of $100 \mathrm{kHz}$. A National Instrument SCXI-1000 system performs as a signal conditioning and a multiplexing platform.

A Pitot tube placed upstream of the plate provides feedback control of the tunnel operating speed by measuring both static and dynamic pressures. An MKS 627B absolute pressure transducer reads the static pressure while an MKS Type 120 transducer monitors the dynamic pressure. The temperature in the tunnel is measured using an Omega DP119 electronic thermometer.

\subsection{Flat Plate Alignment}

Before the experiments begin, the flat plate is aligned so that a zero pressure gradient Blasius boundary layer is established. Boundary layers develop on both the plate and the front wall of the test section and, if not compensated for, reduce the effective flow area on the test side and produce a favorable pressure gradient. Therefore, it is necessary to align the plate so that the area between the test side of the plate and the front test section wall increases slightly in the streamwise direction. This arrangement compensates for boundary layer growth. However, the slight angle of attack can cause the stagnation streamline to impinge on the back side of the plate and cause separation on the test side. This is undesired because it may yield to unsteady separation on the test side that can cause turbulence. A trailing edge flap is implemented in order to prevent this by forcing stagnation to be on the test side of the plate. 
The most sensitive measure for verifying that a zero pressure gradient boundary layer exists is the shape factor, $H$, the ratio of the displacement thickness, $\delta^{*}$ to the momentum thickness, $\theta$. These non-dimensional parameters are defined as follows:

$$
\delta^{*}=\int_{0}^{\infty}(1-U) d y \text { and } \theta=\int_{0}^{\infty} U(1-U) d y
$$

where $U(y)$ is the streamwise basic state velocity non-dimensionalized by the freestream velocity, $U_{\infty}$. For a zero-pressure gradient Blasius boundary layer, the shape factor $H=\delta^{*} / \theta=2.59 . H$ values are obtained at numerous $x$ and $z$ locations in the experimental domain and alignment is performed using adjustment screws on four corners such that they lie within the \pm 0.04 range that is considered to be acceptable (Saric 1996).

An accurate measure of the freestream speed on the test side of the plate (which differs slightly from the Pitot control speed) is also obtained using $\delta^{*}$ and $\theta$. The analytical solution of $U(y)$ for a Blasius boundary layer is known and yields:

$$
\delta^{*}=1.7208 \sqrt{\frac{\left(x-x_{v l e}\right)}{\mathrm{Re}^{\prime}}} \text { and } \theta=0.664 \sqrt{\frac{\left(x-x_{v l e}\right)}{\mathrm{Re}^{\prime}}}
$$

where $x_{v l e}$ is the virtual leading edge location and $\mathrm{Re}^{\prime}=U_{\infty} / v$ is the unit Reynolds number. Once the displacement and momentum thicknesses are calculated from data, a least squares fit is performed to the data using the above functions and $x_{v l e}$ and $\mathrm{Re}^{\prime}$ are obtained. The averages of these two fits are taken and the actual tunnel speed is then calculated using $\mathrm{Re}^{\prime}$ and $v$ which is a function of the pressure and temperature. The error of these fits gives the uncertainty of the effective boundary-layer edge velocity and 
the virtual leading edge. Sample graphs for the shape factors, displacement and momentum thicknesses are presented in Figure 13.

\subsection{Disturbance Generation}

In the present study, it is of utmost importance to generate controlled TS waves and transient disturbances with specific frequencies and wavenumbers. It is desirable to have large transient disturbances because these provide larger modulation of the TS waves and will enable more reliable measurements of any possible interactions. Additionally, it is also desirable to have larger TS wave amplitudes so that the Stokes waves' (sound waves) amplitudes compared to the TS waves are negligible and don't contaminate the TS measurements. Moreover, larger TS amplitudes provides breakdown within the experimental domain. Therefore, techniques available to generate these two types of disturbances are investigated and the best approach is selected as described below.

TS waves are created using the interaction of freestream acoustic forcing and a $2 \mathrm{D}$ roughness strip. This combination is known to be very effective at producing TS waves and it has been extensively studied both experimentally and analytically (Saric et al. 2002). The $2 \mathrm{D}$ roughness strip consists of stacked several layers of double sided tape with a certain thickness and width. The tape is applied with great care to avoid spanwise variations of the tape thickness and air bubbles between the layers. Sound waves are generated using a speaker placed 1 meter upstream of the wind tunnel entrance. The speaker is connected to a signal generator that is capable of providing single frequency sine waves at a variety of amplitudes. Data acquisition is triggered using the generator's 
reference signal and temporal data are phase-lock averaged in time. TS wave amplitudes are related both the $2 \mathrm{D}$ roughness thickness and the sound amplitude. The thickness, width of the tape and the sound amplitudes are selected as described in the following section.

Transient growth can be generated both by 3D surface roughness (distributed or controlled) or freestream turbulence. In the present study, an array of 3D roughness elements is implemented because of numerous advantages it provides. First, a roughness array enables spatial phase locked averaging of spanwise periodic disturbances and this reduces undesired steady, random disturbances. Additionally, an array permits precise control of the spanwise disturbance wavenumbers. Finally, a roughness array consisting of several 3D elements creates transient growth that originates at a single known streamwise location. The transient growth that results from a roughness array is related to spacing, diameter and thickness of the roughness elements. The selection of all these parameters is described in the following section.

\subsection{Parameter Selection}

In the present study, it is desired to have as large as possible TS waves and transient disturbances to be able to investigate their interactions unambiguously. Additionally, breakdown should happen within the experimental domain so that the effect of transient disturbances on TS-dominated transition can be investigated. The experimental parameters to be selected with above in mind that pertains to the generation of TS waves and transient growth are as follows: 
i) freestream speed $\left(U_{\infty}\right)$, acoustic forcing frequency $(f)$ and acoustic amplitude

ii) streamwise location $\left(x_{\text {strip }}\right)$ and thickness $\left(k_{\text {strip }}\right)$ of the 2D roughness strip

iii) streamwise location $\left(x_{k}\right)$, thickness $(\mathrm{k})$, diameter $(d)$ and spacing $\left(\lambda_{k}\right)$ of the $3 \mathrm{D}$ roughness elements

Figure 9 shows a sketch of the experimental setup and disturbance generator parameters. The experimental parameters are selected to optimize a number of sometime conflicting criteria. In the present study, three different experimental configurations are investigated. The parameters in each of the three configurations are summarized in Table 1; criteria used to select these criteria are given below.

\begin{tabular}{|c|c|c|c|}
\hline Parameter & Configuration I & Configuration II & Configuration III \\
\hline$U_{\infty}(\mathrm{m} / \mathrm{s})$ & $16.4 \pm 0.5$ & $19.6 \pm 0.5$ & $17.5 \pm 0.9$ \\
\hline$x_{\mathrm{vle}}(\mathrm{m})$ & $\sim 0$ & $\sim 0$ & $\sim 0$ \\
\hline$f(\mathrm{~Hz})$ & 142 & 185 & 195 \\
\hline$F$ & $53 \pm 3$ & $48 \pm 2$ & $64 \pm 7$ \\
\hline$x_{I}(\mathrm{~m})$ & 0.34 & 0.36 & 0.26 \\
\hline$x_{I I}(\mathrm{~m})$ & 1.32 & 1.24 & 0.91 \\
\hline$N_{I I}$ & 4.48 & 4.93 & 3.41 \\
\hline$N_{x=0.9 \mathrm{~m}}$ & 3.06 & 3.77 & 3.41 \\
\hline
\end{tabular}

Table 1: General experimental parameters. 
In selecting the parameters given in Table 1, the first task was to select the freestream speed $\left(U_{\infty}\right)$, acoustic forcing frequency $(f)$ and acoustic amplitude. Linear stability theory predicts the growth of TS waves for a given non-dimensional frequency $F$ as:

$$
F=2 \pi f v / U_{\infty}{ }^{2} \cdot 10^{6}
$$

Each $F$ has a particular Branch I and II points as indicated in Figure 3. It is desirable to maximize the growth factor $N$ that is defined as an integral of the spatial growth rate $\alpha_{i}$ as

$$
N=\ln \left(A / A_{o}\right)=\int_{R_{I}}^{R_{I}}-2 \alpha_{i} d R
$$

In this expression $A_{o}$ is the TS amplitude at Branch I and $A$ is the TS amplitude at a streamwise location given by

$$
A=\int_{0}^{\infty}|\hat{u}| d y
$$

where $|\hat{u}|$ is the amplitude of the TS wave at a certain dimensional height $y$ above the plate (for the first configuration, the above integration is done with respect to $\eta$ ). The units of $A$ are not reported in the present study because it is not recognizable and more importantly it is not relevant to the scope of this study. In all the experiments amplitudes are calculated similarly and compared to each other. In order to give an idea of its physical meaning, $A=9.09 \cdot 10^{-2}$ approximately corresponds to fluctuations of $4 \% U_{\infty}$. Roughly speaking, lower frequencies $F$ have larger $N$ factors. However, the streamwise locations of Branch I and II points ( $x_{I}$ and $x_{I I}$ respectively) also increase with decreasing $F$ and only a limited plate length is available. Additionally, as shown in Fig. 10, the TS waves are unstable across a range of frequencies for a given Reynolds number and the forcing frequency must be in that range. However, there are other frequencies such as the 
natural resonance frequency of the probe holders and care is taken not to force at a frequency coinciding with one of those. Therefore, the forcing frequency and the tunnel speed are selected such that the $N$ factor is maximized while keeping both the neutral stability points within the experimental domain and within the unstable frequency range.

TS wave amplitudes are proportional to the acoustic amplitude therefore it is desirable to have large acoustic amplitudes. On the other hand, large acoustic amplitudes may contaminate TS wave amplitude measurements in the boundary layer due to Stokes' waves especially upstream where TS wave amplitudes are small. In the present study, achieving larger TS waves dominated over the concerns of Stokes' wave contamination because the plate is relatively short to achieve breakdown within the experimental domain of up to $x=0.9 \mathrm{~m}$. Therefore, the acoustic forcing amplitude is selected to be almost the largest amplitude the signal generator can provide (9 VPP). (This setting was changed in cases where necessary to match the reduced receptivity of TS waves when transient disturbances are present.) Table 1 summarizes the above parameters selected for the three configurations. All distances are with respect to the physical leading edge of the plate.

Secondly, the location of the $2 \mathrm{D}$ and $3 \mathrm{D}$ roughness elements are determined. It is desirable to match the $2 \mathrm{D}$ roughness strip location $\left(x_{\text {strip }}\right)$ with Branch I $\left(x_{I}\right)$ because this provides the largest initial disturbance amplitude for a given acoustic amplitude. The location of the 3D roughness elements $\left(x_{k}\right)$ are selected following two criteria. First, the location of minimum transient disturbance amplitude is desired to coincide with the Branch I point. This is done so that the effect of transient growth on the receptivity of the TS waves at the 2D roughness strip is minimal. Secondly, it is also desirable to match the 
location of the maximum transient growth location with the maximum TS wave growth rate location (roughly of the midpoints between Branches I and II) to maximize the interactions at that location. The measure of transient disturbance amplitudes used for this purpose is the total stationary disturbance energy $E_{\mathrm{rms}}$, given by

$$
E_{r m s}=\int_{0}^{\infty} U^{\prime}{ }_{r m s}^{2} d \eta
$$

where $U_{r m s}^{\prime}$ is the root-mean-square value of the deviations from the Blasius basic state. Typical $\mathrm{E}_{\mathrm{rms}}$ variations are shown in Fig. 11 which has been adapted from Ergin and White (2005). It is not possible to exactly satisfy these two criteria however a trial and error procedure is carried out to the best extent possible. Another measure of transient disturbance amplitudes created is based on local maxima and minima of the streamwise velocity deviation $U^{\prime}(y, z)=U(y, z)-U_{B L}(y)$. It is defined following Andersson et al. (2001) as:

$$
A_{s}=\frac{1}{2}\left[\max _{y, z} U^{\prime}(y, z)-\min _{y, z} U^{\prime}(y, z)\right]
$$

Cossu and Brandt $(2002,2004)(\mathrm{CB})$ use this measure to describe the transient disturbance amplitudes in their study. CB predicted that transient disturbances with amplitudes larger than approximately $A_{s}=0.08$ significantly reduce the growth rate of unsteady disturbances and disturbances with amplitudes exceeding $A_{s}=0.2$ can completely stabilize unsteady waves. In the present study, $A_{s}$ values are presented to provide comparisons. Additionally, $A_{s}$ is quoted by Fransson et al. (2005), Gurun and White (2005), Gurun and White (2004) and White et al. (2004). 
Thirdly, both the transient disturbance and TS wave amplitudes are functions of the thicknesses of the 3D roughness element $(k)$ and 2D roughness strip $\left(k_{s t r i p}\right)$, respectively. Roughness amplitude is described using the roughness based Reynolds number, $R e_{k}$ and it is desired to maximize this parameter to produce large disturbances. $R e_{k}$ is defined as

$$
\operatorname{Re}_{k}=U(k) k / v
$$

where $U(k)$ is the velocity at the roughness height, $k$, and $v$ is the kinematic viscosity. The boundary layer in the vicinity of the wall can be represented by a line such that $U(k)$ is approximately $U(k) \approx U^{\prime}(0) . k$ where $U^{\prime}(0)$ is the velocity gradient at the wall. From the Blasius solution, it is known that $U^{\prime}(0)=0.332 U_{\infty} / \delta$. However, when roughness amplitude is too large, the boundary layer is tripped and immediate bypass transition occurs. The threshold $R e_{k}$ value is denoted by $R e_{k, c r i t}$. Test runs are carried out to determine the $R e_{k, \text { crit }}$ value both for $2 \mathrm{D}$ and $3 \mathrm{D}$ elements. For a certain $U_{\infty}$ and strip location, $R e_{k, c r i t}$ for 2D roughness depends on $k_{s t r i p}$ only however, for 3D roughness, it is also a function of the diameter of the elements. It is known that $\operatorname{Re}_{k, c r i t} \approx 600(k / d)^{2 / 5}$ (Klebanoff et al. 1955, Tani 1969) and this correlation is used as a guide. Initially, paper stickers were used to create 3D roughness. Later it was observed that metal roughness elements (brass, bronze) permitted a higher $\mathrm{Re}_{k, \text { crit }}$ to be obtained before bypass. Therefore, for the latter setups, metal elements were implemented. As a final note on the bypass criteria, preventing bypass of $2 \mathrm{D}$ and $3 \mathrm{D}$ roughness elements separately are not sufficient to guarantee that near-critical 2D and 3D elements together will not cause bypass. In certain cases where transient growth is large and the tape is close to bypass, 
the presence of transient growth was observed to induce bypass at the 2D strip. This is thought to be due to the thinning of the boundary layer at locations downstream of the midpoints between the 3D elements.

As a side note, the width of the 2D strip is also found to be a relevant parameter. In the experiments the width of the strip is selected to be either close to $\lambda_{\mathrm{TS}} / 2$ or $\lambda_{\mathrm{TS}}$ where $\lambda_{\mathrm{TS}}$ is the streamwise wavelength of the TS waves, $\lambda_{T S}=2 \pi \delta_{I} / \alpha_{r}$. In the present study, $\lambda_{\mathrm{TS}}$ is around $30 \mathrm{~mm}$ therefore strips of $19 \mathrm{~mm}, 38 \mathrm{~mm}$ and $36 \mathrm{~mm}$ width (commercially available sizes) are implemented. It appears that half the TS wavelength wide tape results in better TS characteristics. Tape width larger than $\lambda_{\mathrm{TS}}$ seems to adversely affect the intermittency estimation procedure explained later in this chapter and is avoided. Table 2 summarizes the location, thickness and the roughness amplitudes of 2D strip and $3 \mathrm{D}$ roughness elements used in three experimental configurations. The distances are the centerline locations for both $2 \mathrm{D}$ strip and $3 \mathrm{D}$ elements. It can be seen that with each configuration higher roughness amplitudes are achieved as the experimental techniques were progressively improved. 


\begin{tabular}{|c|c|c|c|}
\hline Parameters & Configuration I & Configuration II & Configuration III \\
\hline$x_{\text {strip }}(\mathrm{m})$ & 0.291 & 0.261 & 0.228 \\
\hline Strip width $(\mathrm{mm})$ & 19 & 38 & 36 \\
\hline$k_{\text {strip }}(\mu \mathrm{m})$ & 564 & 564 & 628 \\
\hline $\mathrm{Re}_{\text {ktape }}$ & 212 & 273 & 328 \\
\hline$x_{k}(\mathrm{~m})$ & 0.15 & $0.2,0.19$ & 0.16 \\
\hline$k(\mu \mathrm{m})$ & $406.4,508$ & $406.4,500$ & 508 \\
\hline $\mathrm{Re}_{k}$ & 153,238 & 168,260 & 246 \\
\hline
\end{tabular}

Table 2: 2D strip and 3D roughness element thicknesses, locations and thicknesses

Thirdly, the spacing of the $3 \mathrm{D}$ roughness elements, $\lambda_{k}$ is determined. Optimal disturbance theory (Andersson et al. 1999, Luchini 2000 and Tumin and Reshotko 2001) predicts that the optimal spanwise wavenumber is $\beta=0.45=2 \pi \delta_{x \max } / \lambda_{k}$ where $\delta_{x \max }$ is the boundary layer thickness at the location of the maximum TS wave growth rate. Even though the disturbances created by surface roughness are not optimal, optimal theory serves as a guide for the selection of the spanwise spacing. Once the TS parameters are selected, $\delta_{x \max }$ is known and from this the spanwise spacing, $\lambda_{k}$. In the third experimental configuration, the spanwise spacing of the elements is selected using a different criterion. In that configuration, $\lambda_{k}$ is selected to be on the order of the TS wavelength such that it coincides with the breakdown pattern of the fundamental Klebanoff-type vortices.

Fourthly, the diameter of the 3D roughness elements is selected. Larger diameters tend to cause larger transient growth. However, the maximum thickness that can be 
attained without bypassing, $R e_{k c r i t}$ decreases as the diameter increases. Therefore, there is a balance between these two and for all the experiments performed, the diameters are half of the spanwise spacing, $\lambda_{k} / 2$. Table 3 summarizes the spacing and diameter of the $3 \mathrm{D}$ roughness elements used in three configurations.

\begin{tabular}{|c|c|c|c|}
\hline Parameters & Configuration I & Configuration II & Configuration III \\
\hline$\lambda_{k}(\mathrm{~mm})$ & 11.43 & 11.43 & 28 \\
\hline$d=\lambda_{k} / 2(\mathrm{~mm})$ & 5.715 & 5.715 & 14 \\
\hline
\end{tabular}

Table 3: Spacing and diameter of 3D roughness elements

\subsection{Experimental Procedure}

To begin data acquisition at a certain $x$ location, the hotwire traverse is positioned at the minimum $z$ location of the measurements at a $y$ location just outside the boundary layer. The numerical value of the $y$ coordinate is arbitrary at this point and is set to be $y=0$. After data is recorded at a certain location, traverse moves closer to the plate with a certain $y$ step. The step size is calculated by multiplying an initial $y$ step by the boundary layer velocity normalized by the freestream velocity to the power 1.75 . This results in a $y$ step that becomes progressively smaller as the wire approaches the plate. This provides more data points lower in the boundary layer where good spatial resolution is necessary. The wire stops moving closer to the plate when a certain non-dimensional boundary layer velocity is reached. This is essential to prevent the boundary layer wire from losing heat 
to the plate and breaking. For the present study, values of $0.12 \%$ to $0.20 \%$ of $U_{\infty}$ are used. Once the traverse completes one dive into the boundary layer, it moves out in the $+y$ direction and then moves in the $+z$ direction to the next spanwise location. In the present study, the spanwise step is selected to be $1 / 8$ of the spanwise spacing of the $3 \mathrm{D}$ roughness elements to have enough spanwise points (also power of 2) in the span to do a spatial Fast Fourier transform. For the baseline cases where there is no modulation due to $3 \mathrm{D}$ roughness, the spanwise step is $10 \mathrm{~mm}$ in Configurations I and II and is the same as the modulated case in Configuration III. The hotwire movement pattern continues until certain number of spanwise wavelengths are covered. In the present study, 8 or 5 wavelengths are obtained.

At each location, velocities are acquired in multiple samples of 350, 1800 or 1200 data points. In the present study, 8,12 or 36 samples are obtained for the following ranges of the dimensionless freestream velocity $U_{\infty}>0.95,0.49<U_{\infty}<0.95$ and $U_{\infty}<0.49$ respectively. Temporal phase locked averaging is performed over the samples to improve the signal quality.

The $y$ location and the dimensionless velocity at each measurement location are recorded. This information is used to estimate the wall location at each $z$ and then convert the $y$ coordinate of each measurement to wall normal coordinate, $\eta$. The slope of the boundary layer $\mathrm{U}$ velocity below $35 \%$ of $U_{\infty}$ is almost a straight line and a linear fit to zero velocity is performed to those points to yield the wall location for each spanwise location. Later, a second degree polynomial in $z$ is fit to the wall location estimates for select spanwise points. In the presence of transient growth, the steady flow field most resembles Blasius flow at span locations between roughness elements. Therefore, the 
wall estimates for these points are used in the least square fit for $y_{\text {wall }}(z)$. Without transient growth, the steady flow field for all spanwise locations is Blasius and all wall estimates are used.

\subsection{Intermittency Estimation}

A primary objective of these experiments is to provide a quantitative measure of any transition delay provided by transient disturbances. This is accomplished by estimating the turbulent intermittency, $\gamma$, throughout the transition region. Intermittency is defined as the fraction of the time a flow is turbulent; $\gamma=0$ is fully laminar while $\gamma=1$ is fully turbulent. If a detector function $f(t)$ is used that gives $f=1$ when a flow is turbulent and $f=0$ otherwise then

$$
\gamma=\frac{1}{T} \int_{0}^{T} f(t) d t
$$

Two essential decisions need to be made to estimate turbulent intensity. One is the choice of the detector function and the other is the determination of threshold value. Intermittency estimation is a somewhat subjective process and various detector functions and threshold determination methods have been implemented by different researchers with two notable contributions due to Hedley and Keffer (1974) and Kuan and Wang (1990). Hedley and Keffer (1974) use the region of maximum curvature of intermittency versus threshold curve while Kuan and Wang (1990) use the dual-slope method (intermittency versus threshold curve appears as two lines with different slopes when plotted in a semilogarithmic diagram) to determine the threshold value. Both of these techniques were implemented in the present study. However, with the present data, both 
the point of maximum curvature and the change of slope are not always clear. The technique proposed by Fransson et al (2005) was found to be more suitable. This method consists of finding the absolute value of the high-pass-filtered $u^{\prime}$ velocity signal, performing a short-window smoothing of the filtered values and calculating the fraction of time this value exceeds specified threshold value. For each measurement location and condition, the log of this fraction is plotted as a function of the threshold value. As the threshold approaches zero, the intermittency estimate approaches unity and its log approaches zero. For a range of threshold values not including zero, $\log \gamma$ decreases roughly linearly with the threshold fluctuation velocity, $u_{t}^{\prime}$. The point at which the bestfit line to $\log \gamma$ in the linear range passes through $u_{t}^{\prime}=0$ is taken to give the intermittency at a particular point.

For this work the high-pass filter cutoff is selected to be $500 \mathrm{~Hz}$, well above the TS-unstable frequency range and the absolute value of the filtered signal is averaged over three-point windows (periods of 0.6 or 7.2 milliseconds in the present study). Thresholds ranging from $u_{t}^{\prime}=0$ to $0.05 \mathrm{~m} / \mathrm{s}$ are used in the least-squares fit procedure and the fit is performed using $\gamma$ estimates for $u_{t}^{\prime}=0.025$ to $u_{t}^{\prime}=0.05 \mathrm{~m} / \mathrm{s}$.

Figure 12a shows a sample 0.1 seconds long velocity trace measured by the boundary layer hotwire. Two turbulent bursts can be seen. The high-pass-filtered signal is shown in Fig. 12b. The high-frequency bursts corresponding to turbulence appear clearly. The high-pass signal is then smoothed to eliminate spurious zeros (Fig. 12c). At this stage, the turbulent intermittency levels are plotted as a function of the threshold velocity. As the threshold velocity approaches zero from the right, the intermittency rises rapidly due to contamination from electronic noise. To avoid the ambiguity introduced by this, a 
linear fit is performed in the region between the threshold levels mentioned above and the intersection of that line with the abscissa is taken to be the estimated intermittency value. The threshold corresponding to that turbulent intermittency value is then selected to be the threshold. For this sample, it is found to be $u_{t}^{\prime}=0.013 \mathrm{~m} / \mathrm{s}$. As an a posteriori check of the technique, the effective detector function is determined (Fig. 12d) and this is superposed on the original velocity signal (Fig. 12e). This technique effectively captures the turbulent bursts.

Once the intermittency is estimated for each location, the maximum in the wallnormal direction is taken to be the value for each $x$ and $z$. Later, for a given spanwise location (see Fig. 29 for example) $\gamma$ values for all $x$ are fit to a universal intermittency function proposed by Narasimha (1957):

$$
\gamma(x)=1-\exp \left[-\left(x-x_{t}\right)^{2} \frac{n \sigma}{U_{\infty}}\right]
$$

Above, $n \sigma / U_{\infty}$ is assumed to be constant with $n$ and $\sigma$ being the turbulent spot production rate per unit length in the spanwise direction and Emmon's dimensionless spot propagation parameter, respectively. The intermittency levels plotted in the graphs are interpolated using this equation.

\subsection{Data Analysis}

Extensive data analysis is performed in two phases. First, a preliminary analysis is performed in real time as data is acquired and the results are recorded. However, most of 
the analysis is performed later using a different computer so that data acquisition is not delayed by processing.

In the preliminary analysis stage, multiple boundary layer velocity samples are decomposed into their steady and unsteady components as $u(x, y, z, t)=U(x, y, z)+$ $u^{\prime}(x, y, z, t)$. The unsteady part is Fourier transformed in time to obtain the power spectral density (PSD). The Fourier-coefficients at the forcing frequency are extracted from each PSD and these complex values are averaged to reduce their uncertainty. Because data acquired is triggered using the timing signal of the signal generator that drives the acoustic forcing, the imaginary and real parts of the Fourier coefficients are averaged separately and this eliminates fluctuations not associated with the forced acoustic input.

For the intermittency estimation, the first eight samples from each location are extracted and joined together. The procedure described in the previous section is implemented and the resulting intermittency value versus threshold curve is recorded. For each data point, PSD values, non-dimensional mean velocities and the associated $(y, z)$ location are recorded in a data file with a header that contains the date, $x$-location and the environmental parameters.

In the second, offline analysis phase, the steady velocities are decomposed into spanwise-uniform and spanwise varying components $U(x, y, z)=\bar{U}(x, y)+U^{\prime}(x, y, z)$. The spanwise uniform component $\bar{U}(x, y)$ is not the spanwise mean. Instead, it is the mean of the profiles obtained in between the roughness elements where boundary layer most resembles Blasius basic state. The $E_{\mathrm{rms}}$ and $A_{\mathrm{s}}$ values that describes transient disturbance amplitudes are then calculated using $U^{\prime}(x, y, z)$. The displacement and momentum thicknesses as well as shape factors are calculated using $\bar{U}(x, y)$. 
The wall-normal location $y$ is converted into $\eta$ as previously described and the PSD amplitudes are interpolated to a rectangular grid for each spanwise location. PSD values are then averaged across the whole span for the baseline cases and across onewavelength for modulated cases. The square root of averaged PSD values are the TS fluctuation intensities and a wall normal integration is performed to find the amplitudes, $A$. For the modulated cases, TS intensities are Fourier transformed in the spanwise direction to obtain the spanwise modes defined as:

$$
\left|\hat{u}_{F}(x, \eta, z)\right|=\sum_{n=-\infty}^{\infty} \tilde{u}_{n}(x, \eta) e^{2 \pi i n z / \lambda_{k}}
$$

This transformation is done in two halves of the spanwise domain and the two are averaged to reduce the variance of the results. Finally, for each data point, the intermittency versus threshold graph is plotted and the threshold is selected yielding an intermittency value for that data point. 


\section{Chapter 3}

\section{RESULTS AND DISCUSSION}

Numerical simulations performed by Cossu and Brandt $(2002,2004)$ (CB) indicate that the growth of unsteady TS-like disturbances can be suppressed in the presence of optimal transient disturbances. However, this effect has not been conclusively demonstrated in an experiment and, therefore, it remains unclear whether this suppression is physically realizable and, if it is, whether the growth-rate reduction can delay breakdown to turbulence in TS-dominated transition. Therefore, experimental verification of TS growth rate suppression in the presence of transient disturbances is and has been of interest at the Case Wind Tunnel Laboratory. Prior to the present study, Shelley (2004) performed experiments and observed M-shaped modulations of the unsteady disturbances' wallnormal profiles as predicted by $\mathrm{CB}$. However, these experiments did not unambiguously verify growth rate suppression or transition delay. Therefore, the first objective of the present study is to verify the growth rate suppression with physically realizable transient disturbances. The second objective is to investigate this effect as a possible means to delay transition. This chapter is grouped into three experimental configurations designed that progressively achieve these objectives.

\subsection{Configuration I}

The first set of experiments performed as part of this work was conducted between September and December 2004. At that time, the objective was to obtain a baseline case 
of TS waves produced by acoustic forcing and a $2 \mathrm{D}$ roughness strip in a $2 \mathrm{D}$ boundary layer. This baseline was intended to establish wall-normal disturbance profiles and disturbance growth curves that were consistent with linear stability theory as a notransient-growth control case. Once this was established, the second objective was to verify suppressed growth of the modified TS waves in the 3D boundary layer created by introducing stationary transient disturbances using a spanwise array of 3D roughness elements.

In this Configuration $\mathrm{I}, U_{\infty}=16.4 \pm 0.5 \mathrm{~m} / \mathrm{s}$ and $f=142 \mathrm{~Hz}$. At typical laboratory pressure and temperature this results in a nondimensional frequency, $F=53 \pm 3$. The trailing edge of the $19-\mathrm{mm}$-wise $2 \mathrm{D}$ strip is placed at $x=300 \mathrm{~mm}$, slightly upstream of the Branch I point for $F=53$. The strip thickness is $564 \mu \mathrm{m}$ and this gives $\mathrm{Re}_{\text {ktape }}=212$. The sampling rate is $1000 \mathrm{~Hz}$ and the sample size is 512 yielding 0.512 seconds per sample. Two different arrays of 3D roughness elements are created using paper stickers. Both arrays are located at $x=150 \mathrm{~mm}$. One array is $406 \mu \mathrm{m}$ thick which gives $\operatorname{Re}_{k}=153$; the other is $508 \mu \mathrm{m}$ thick which gives $\operatorname{Re}_{k}=238$. The spacing between the elements is $\lambda_{k}=11.43 \mathrm{~mm}$ and the diameter of the elements is $\lambda_{k} / 2$. Measurements are taken at 9 spanwise locations using a $10 \mathrm{~mm}$ step size for the baseline case and at 64 spanwise locations with a step size of $1.43 \mathrm{~mm}$ ( 8 positions per roughness wavelength) for the cases with the $3 \mathrm{D}$ roughness array.

\subsubsection{Baseline Case: TS waves in a spanwise-uniform basic state - I}

To begin the Configuration I baseline experiments, the zero-pressure-gradient basic state Blasius boundary layer is established using measurements of the displacement and 
momentum thicknesses and spanwise-averaged shape factors. These are presented in Fig. 13. In this figure and all subsequent figures that indicate the variation of some quantity with respect to the $x$ direction, $x$ and $R=\operatorname{Re}^{1 / 2}=\left(\operatorname{Re}^{\prime} \cdot x\right)^{1 / 2}$ are interchangeably indicated as equivalent dependent variables. (Transient growth results are more easily understood with respect to the $x$ coordinate while TS growth results are more easily understood in terms of $R$.) Figure 13 indicates that the shape factors lie within the acceptable range. Similarly, Fig. 14 shows that the wall-normal profile of the TS wave produced by acoustic forcing is in agreement with the profile predicted by the linear theory.

TS disturbance profiles from all $x$ are integrated to obtain the $N$-factor curve shown in Fig. 15. The disturbance growth is in agreement with the linear theory prediction except at the most upstream and downstream locations. The deviation at the downstream points is due to turbulence arising from TS wave breakdown and the growing importance on non-linearities. The deviation upstream is due to contamination of the TS disturbance signal measurement by the presence of Stokes wave (acoustic boundary layer) fluctuations that are associated with the freestream acoustic forcing. At $R=845(x=700 \mathrm{~mm})$, the TS wave is sufficiently large that the sound wave amplitude is only $3 \%$ of the TS wave amplitude near $\eta=1$. However, at the upstream points close to Branch I the TS waves are small and the Stokes waves are of roughly equal amplitude. This contamination by the Stokes waves only affects the ability to measure the TS wave but does not affect the TS wave because both disturbances are of small amplitude and do not interact.

In spite of the deviations of the measurements from linear theory predictions at the upstream and downstream ends of the measurement domains, TS waves do indeed appear to behave as predicted by linear theory across most of the measurement domain. 
Moreover, the deviations are understood not to affect the outcome of the experiments. Therefore, it is evident that TS wave consistent with the linear theory can be produced using acoustic forcing and 2D roughness strip and that the experiments may proceed.

\subsubsection{Spanwise Modulated Basic State - II}

Once the baseline case is established, transient stationary disturbances are introduced using the spanwise arrays of roughness elements at $x=150 \mathrm{~mm}$. One set of measurements uses elements with $\mathrm{Re}_{k}=153$, a second set uses $\mathrm{Re}_{k}=238$. The total stationary disturbance energy, $E_{\mathrm{rms}}$ and disturbance amplitude $A_{s}$ variations with respect to the streamwise location are given in Figs. 16 and 17 for both the modulated cases. It is observed that with the exception of the measurements obtained upstream of and directly above the $2 \mathrm{D}$ roughness strip, $x=291 \mathrm{~mm}$, transient disturbances are strictly decaying in the measurement domain. It is unclear whether the very high-amplitude transient disturbance observed above the $2 \mathrm{D}$ roughness strip is associated with a temporary but large increase in the disturbance amplitude at that location or is simply a measurement artifact associated with less-certain wall location estimates above the strip. Regardless, it is observed that the measurements downstream of the strip are self-consistent and are consistent with other measurements of transient disturbance behavior in the facility. In

particular, the disturbance energies (amplitudes) scale as $\operatorname{Re}_{k}{ }^{2}\left(\operatorname{Re}_{k}\right)$ as has been reported by White and Ergin (2003) and White et al. (2005).

The spanwise modulation of the displacement thickness and the steady streamwise velocity by the transient disturbances are shown in Figs. 18 and 19. The phase-locked velocity contours correspond to non-dimensional steady velocity levels 
with $10 \%$ increments. In Fig. 19 and other similar figures, the spanwise $z$ axis is normalized by the spanwise spacing of the $3 \mathrm{D}$ roughness array, $\lambda_{k}$. Locations behind the roughness elements' centers are $z=0$ and locations between the elements' centers are $z=$ \pm 0.5 . As the transient disturbance amplitude increases, the modulation of both the displacement thickness and the velocity becomes more pronounced, that is the variations of these quantities across the span in Fig. 19 with $\mathrm{Re}_{k}=238$ are more distinct than of those in Fig. 18 with $\mathrm{Re}_{k}=153$. It can be observed that the boundary layer is somewhat accelerated in the high speed region (i.e., has a smaller boundary-layer thickness) than in the low speed region streak. High speed streaks originate behind roughness elements and low-speed streaks originate between elements.

The evolution of the unsteady TS-wave-like-disturbances is plotted in Figs. 20 and 21. The predicted M-shape modulations can easily be seen in the low-speed streak region. Comparing Fig. 20 and 21 shows that this effect becomes more pronounced with increasing transient disturbance amplitude. Additionally, the M-shape modulations become more pronounced as the unsteady disturbance amplitudes grow, that is as $R$ increases in these figures. No M-shaped modulations are observed in the unsteady disturbance profiles in the high-speed region. These profiles still resemble classical TS wave profiles. It is observed that the amplitudes of the unsteady waves are reduced as the transient disturbance amplitude increases by comparing Fig. 20 and 21. On a superficial level, this could be taken as an indication that the desired disturbance reduction has been achieved. However, the reduction of the unsteady disturbances' amplitudes is largely due to a reduction of these disturbances' initial amplitudes (i.e., a reduction of the receptivity) when the 3D roughness elements are introduced. For the Configuration I setup, the 
unsteady disturbances' initial amplitude, $A_{0}$, is found to be only $60 \%$ and $50 \%$ of the nonmodulated $A_{0}$ for the $\mathrm{Re}_{k}=153$ and $\mathrm{Re}_{k}=238$ roughness arrays, respectively.

In addition to comparing the behavior in the high- and low-speed-streak regions, it is also useful to consider the spanwise modes of the unsteady disturbances as defined in Eqn. (2.11). To examine these, the unsteady disturbance profiles are Fourier transformed in the span direction. Figure 22 shows the spanwise invariant, fundamental and the second harmonic mode of the unsteady disturbances at $R=934(x=850 \mathrm{~mm})$. The spanwise invariant mode represents the spanwise-averaged profile of the unsteady waves across the span. The fundamental mode describes the disturbance's variation on the spanwise wavelength $\lambda_{k}$ and the second harmonics represents variations on $\lambda_{k} / 2$. Superposition of these modes gives the unsteady profiles at each spanwise location. Higher harmonics are not observed to be significant in Configuration I. This is somewhat different than what was observed by Shelley (2004) who observed significant $\lambda_{k} / 3$ modes when the $3 \mathrm{D}$ roughness diameter was $1 / 3 \lambda_{k}$.

The spanwise invariant mode makes the largest contribution to the total disturbance and bears the closest resemblance to the classical TS wall-normal profile. The M-shapes in the wall-normal profiles arise from the fact that the fundamental mode is adds to and subtracts from the spanwise invariant mode in the high- and low-speedstreak regions respectively. In constrast to this, $\mathrm{CB}$ predicted M-shape modulations to be significant in the spanwise invariant mode (Fig. 4). Interestingly, this was not the case in the present experiments. This might be related to the fact that the transient disturbances created in the present experiments are sub-optimal as opposed to optimal disturbances used by $\mathrm{CB}$. Another explanation might be that the transient disturbance amplitudes created in the experiments were much lower than those considered by $\mathrm{CB}$. The 
experimental transient disturbance amplitudes are approximately $A_{s}=0.05$ and $A_{s}=0.03$ as compared to the lowest amplitude $\mathrm{CB}$ reported $\left(A_{s}=0.14\right)$. A third explanation might be that the combination of the $3 \mathrm{D}$ elements and acoustic waves produce fluctuating disturbances with the fundamental spanwise periodicity and that these superpose on the originally $2 \mathrm{D}$ waves produced at the strip.

Figures 23 and 24 show $N$-factor curves of the unsteady disturbances that grow in the high- and low-speed streaks. The growth rate of the unsteady waves (i.e., the slope of $N$-factor curve) is slightly lower in the low-speed region than in the high-speed region and the difference becomes more pronounced with increasing transient disturbance amplitudes. Even though the difference is subtle, it appears to verify that introducing transient disturbances does reduce the growth rate of unsteady waves.

As a summary, these experiments verify in a preliminary way that transient disturbances do create M-shape modulations in the wall-normal profiles of unsteady disturbance waves and that the growth rate of these waves is reduced. The growth rate reduction is not nearly as large as the reductions obtained by $\mathrm{CB}$ but this is thought to be due to the lower transient disturbance amplitudes of the present experiments. In contrast to what was predicted by $\mathrm{CB}$, the spanwise decomposition reveals that M-shape modulations are not in the spanwise invariant mode but instead arise from the fundamental and harmonic spanwise modes.

Additionally, it is found that the receptivity of the unsteady waves is significantly lower in the presence of transient disturbances than in the baseline case. This was an unexpected result. With regards to the transition delay, both reduced growth rate and receptivity mean lower unsteady wave amplitudes at given streamwise location which may result in transition delay. However, the receptivity reduction is a different 
mechanism than what was considered by $\mathrm{CB}$ and it is not clear whether this reduction is unique to this configuration or is a more universal effect. Therefore, to be able to isolate the effect of the growth rate reduction on transition, the receptivity reduction should be accounted for by changing (increasing) the initial amplitude of the TS-like disturbances so that the initial amplitudes in the baseline no-roughness case and modulated cases are equal. Additionally, because the results of Configuration I do not identify a transition location in an unambiguous, quantitative way, intermittency measurements should be performed to investigate the breakdown behavior.

\subsection{Configuration II}

The results of Configuration I were instructive in that certain features predicted by $\mathrm{CB}$ were validated while others were not but, more importantly, requirements for an improved experiment were developed. This led to a second set of experiments performed between February and April, 2005. At that time, results from the first configuration showed that transient disturbances modulate wall-normal profiles of unsteady waves. However, the growth rate reduction realized in the experiment was not sufficiently strong to produce unambiguous results, especially with regards to any possible transition delay. The second setup, Configuration II, was designed to unambiguously verify the growth rate suppression using larger transient disturbances. The Configuration II experiments also included measurements of turbulent intermittencies across the experimental domain to provide more quantitative data on the transition location.

In order to achieve these objectives, it was necessary to create larger transient disturbances and unsteady waves by increasing the roughness amplitudes of both the 2D 
roughness strip and 3D roughness elements. Additionally, the dimensionless frequency was reduced from $F=53$ to $F=48$ to produce larger growth rate of unsteady waves.

In this configuration, $U_{\infty}=19.6 \pm 0.5 \mathrm{~m} / \mathrm{s}$ and $f=185 \mathrm{~Hz}$. At typical laboratory conditions this results in $F=48 \pm 2$. The trailing edge of the $2 \mathrm{D}$ strip with a width of 38 $\mathrm{mm}$ is placed at $x=280 \mathrm{~mm}$ which is slightly upstream of the Branch I point for $F=48$.

The strip thickness is $564 \mu \mathrm{m}$ and gives $\mathrm{Re}_{\text {ktape }}=273$. The sampling rate is $5000 \mathrm{~Hz}$ and the sample size is 1800 yielding 0.36 seconds per sample. Two different arrays of elements with $406.4 \mu \mathrm{m}$ and $500 \mu \mathrm{m}$ thickness are implemented. The first array of $\mathrm{Re}_{k}=168$ is placed at $x=200 \mathrm{~mm}$ and the second array of $\mathrm{Re}_{k}=260$ is placed at $x=190 \mathrm{~mm}$. The spacing between the elements is $\lambda_{k}=11.43 \mathrm{~mm}$ and the diameter of the elements is $\lambda_{k} / 2$. The measurements are taken at 9 spanwise locations with a $10 \mathrm{~mm}$ step size for the baseline case and at 64 spanwise locations with a step size of $1.43 \mathrm{~mm}$ for the modulated cases.

\subsubsection{Baseline Case: TS waves in a spanwise-uniform basic state - II}

As in the previous configuration, the flat plate alignment is verified using the displacement and momentum thickness and shape factors (Fig. 25). In Fig. 25, a sharp decrease in $H$ is observed at the most downstream locations as the boundary layer becomes turbulent at those locations. Excluding the downstream points, the average shape factor is 2.54 . This indicates that the flow is slightly accelerated relative to a true zero-pressure-gradient boundary layer. 
TS waves should be uniform in the span direction. The actual spanwise uniformity the TS amplitudes' at the waves' two amplitude peaks $\eta=1$ and $\eta=5$ at $R=860(x=600 \mathrm{~mm})$ is given in Fig. 26. The figure indicates that a spanwise variation of $25 \%$ of the spanwise mean TS amplitude is present without transient disturbances. While a smaller variation would be preferable, this is not an unreasonably large variation given the difficulties in establishing a perfect basic state and disturbance generation scheme. The wall-normal TS profiles are observed to be in good agreement with linear theory as is shown in Fig. 27. Spanwise-averaged TS amplitudes are used to construct the $N$-factor curve shown in Fig. 28. The $N$-factors are in agreement with linear theory until about $R=930(x=700)$ at which point turbulent bursts begin to appear.

In order to investigate breakdown to turbulence, the turbulent intermittencies are measured as described in Chapter 2. A typical intermittency variation versus $x$ is shown in Fig. 29 for one spanwise location. This variation is in reasonably good agreement with Narasimha's universal gamma function. This function is used at all spanwise locations and an interpolation of the best-fit function at each $z$ is used to estimate the streamwise location at which the intermittencies are $10 \%, 30 \%$ and $50 \%$. These contours of these intermittency levels in $x$ and $z$ are given in Fig. 30 and show impressive uniformity across the span. As mentioned above, the breakdown approximately begins at $x=700 \mathrm{~mm}$ where the TS amplitude is $A=0.18$.

TS waves consistent with linear theory exist in the baseline case for Configuration II and breakdown is observed within the experimental domain. Transient disturbances may now be introduced. 


\subsubsection{Spanwise Modulated Basic State - II}

Two different spanwise arrays of $\mathrm{Re}_{k}=168$ and $\mathrm{Re}_{k}=260$ are used to modulate the baseline case. Variations in $E_{\mathrm{rms}}$ and $A_{s}$ with respect to $x$ is given in Figs. 31 and 32 for both arrays. The $\operatorname{Re}_{k}=168$ case gives approximately $A_{s}=0.05$ in the experimental domain while $\operatorname{Re}_{k}=260$ gives $A_{s}=0.08$. The spanwise phase-locked velocity contours are given in Figs. 33 and 34. The high and low speed streaks are clearly stronger than in Configuration I where $A_{s}$ was roughly 0.03 and 0.05 in the measurement domain for the two roughness amplitudes.

Figures 35 and 36 show the M-shape modulations in the low-speed region that are consistent with the previous configuration. Again, it is observed that the M-shapes become much more pronounced with the higher transient disturbance amplitudes. The spanwise modes for both of the modulated cases at $R=946(x=725 \mathrm{~mm})$ are plotted in Fig. 37. The spanwise invariant mode is almost 4 times larger than the other modes. As before, no M-shape modulations are observed in the spanwise invariant mode.

Integrating the disturbance profiles at every $z$ location in the wall-normal direction and averaging these amplitudes across the span gives a measure of the overall unsteady disturbance amplitude and its growth. The spanwise averaging is intended to lump the different amplitudes in the high- and low-speed-streak regions into a single amplitude metric because growth in either region is likely to be sufficient to cause transition. The growth of the overall unsteady disturbance energy is shown in Figs. 38

and 39 for the two modulated cases. The growth rate of the unsteady disturbances is strongly reduced relative to the baseline configuration, especially when the largeramplitude $\mathrm{Re}_{k}=260$ roughness array is used. 
Consistent with the finding of Configuration I, it is found that the receptivity of the unsteady disturbances is reduced when a roughness array is present. The effective initial amplitudes are $50 \%$ and $40 \%$ of the non-modulated case for $\mathrm{Re}_{k}=168$ and $\mathrm{Re}_{k}=260$ cases, respectively. Given the results of the first configuration, it seems that the receptivity of unsteady waves gradually decreases as transient disturbance amplitudes increases. This suggests that there is a fundamental and consistent effect of transient disturbances and/or the presence of a 3D roughness array on unsteady wave receptivity at a $2 \mathrm{D}$ roughness strip. Interestingly, a similar effect has been observed by another group at $\mathrm{KTH}$ who used a vibrating ribbon as a TS wave generator (Cossu, private communication, 2005).

To account for the decreased receptivity, a second set of TS measurements are performed without roughness modulation. In these tests, the amplitude of the acoustic forcing is increased so that the unsteady disturbance amplitudes in the non-modulated case and the modulated cases match at $R=912(x=675 \mathrm{~mm})$. No other parameters were changed and the unmodulated TS waves are of similar quality of those obtained at higher forcing amplitudes. Obviously, it is not possible to match the unsteady-disturbance amplitude all the points in the measurement domain because the growth rates of the nonmodulated disturbances are larger than their modulated counterparts. The matching point is somewhat arbitrary and is chosen so that it is close to the middle of the experimental domain where well resolved amplitude measurements are possible and the amplitude match can be performed with some confidence at the outset of the measurements. In retrospect, this choice was unfortunate because the effect of the reduced growth rate in the downstream portion of the experimental domain was somewhat diminished because 
only a short streamwise distance downstream of the matching point was available for comparison.

In order to compare the breakdown of modulated unsteady waves with the nonmodulated cases, intermittency measurements are performed. As before, universal intermittency curves are fit to the intermittency data for each $z$ location in the domain. The fits are not as good as for the non-modulated case but are still useful as a means of interpolating to specific intermittency $(\gamma)$ values for the purposes of plotting intermittency contours across $x$ and $z$. Figures 35 and 36 show the contours of $10 \%$ intermittency for the modulated and their amplitude matched non-modulated cases. It can be seen that in the presence of transient disturbances, this contour level is slightly upstream of TS amplitude matched case. The streamwise points of the initial breakdown are $x=725 \mathrm{~mm}(R=946)$ and $x=750 \mathrm{~mm}(R=962)$ for the $\mathrm{Re}_{k}=168$ and $\mathrm{Re}_{k}=260$ cases, respectively. The spanwise-averaged unsteady disturbance amplitudes at these initial breakdown locations are $A=0.143$ and $A=0.118$, respectively. These amplitudes are lower compared to unmodulated TS wave breakdown amplitude, $A=0.18$. Another observation from the $\gamma$ contours is that there is not a clear connection between the spanwise locations of initial breakdown and features of the unsteady disturbance profiles for either of the modulated cases.

Table 4 gives the breakdown location and unsteady disturbance amplitudes just prior to breakdown for all of the Configuration II cases. The breakdown location moves downstream as the transient disturbance amplitude increases. However this is largely due to decreased receptivity rather than growth rate reduction. The amplitude-matched TS waves partially account for the effect. It is observed that for each amplitude matched case, the disturbances in the spanwise-modulated basic state breakdown at somewhat 
lower amplitude than their amplitude-matched non-modulated counterparts. This effect is not sufficiently strong to draw any clear conclusions but is critical to understand better if the transient disturbances are to be used to control or delay transition.

\begin{tabular}{|c|c|c|}
\hline & Breakdown Location & Breakdown Amplitude \\
\hline Baseline Case & $x=700 \mathrm{~mm}$ & 0.180 \\
\hline $\operatorname{Re}_{k}=168$ Modulated Case & $x=725 \mathrm{~mm}$ & 0.143 \\
\hline $\begin{array}{c}\text { Non-modulated case amplitude- } \\
\text { matched to } R e_{k}=168\end{array}$ & $x=750 \mathrm{~mm}$ & 0.151 \\
\hline $\mathrm{Re}_{k}=260$ Modulated Case & $x=750 \mathrm{~mm}$ & 0.118 \\
\hline $\begin{array}{c}\text { Non-modulated case amplitude- } \\
\text { matched to } \mathrm{Re}_{k}=260\end{array}$ & $x=775 \mathrm{~mm}$ & 0.138 \\
\hline
\end{tabular}

Table 4: Breakdown locations and unsteady disturbance amplitudes at breakdown for all Configuration II cases

As a summary of the results of this configuration, in the presence of transient disturbances, M-shape modulations in the unsteady disturbance's wall-normal profiles are observed and it is unambiguously verified that the growth rate of these disturbances is reduced. The spanwise decomposition of these disturbance profiles revealed that there are no M-shape modulations in the spanwise invariant mode similar to configuration I. Additionally, the receptivity is found to be reduced that becomes even more pronounced with increased transient disturbances. Although inconclusive, the results suggested that when the receptivity reduction is accounted for, the breakdown amplitudes of unsteady waves are reduced in the presence of transient disturbances. This has an interesting 
implication in terms of transition delay. If the breakdown amplitudes are reduced, even though the growth rate of unsteady waves is reduced, it may not provide an advantage in terms of breakdown location. So, in the presence of transient disturbances, three effects have been observed: the growth rate, receptivity and breakdown amplitude of unsteady waves are all reduced. It is not clear which of these effects is the most important and a third experimental configuration is designed to further eliminate the effect of reduced receptivity and investigate the breakdown amplitudes of unsteady waves with reduced growth rate.

\subsection{Configuration III}

The third set of experiments performed as part of this work was conducted between June and August, 2005. Results from the previous configurations showed that the transient disturbances modulate the wall-normal profiles of unsteady waves and reduce their growth rate. Additionally, the breakdown location is moved downstream but that this effect is largely related to reduced receptivity. Matching this reduced receptivity and investigating breakdown hinted a very interesting phenomenon. It was observed that in the presence of transient disturbances, the breakdown amplitude of the unsteady waves is reduced. However, the observed effect was not sufficiently strong to be considered conclusive. Therefore, a third setup denoted Configuration III was designed to investigate the breakdown amplitudes of unsteady waves with and without transient disturbances.

In addition to reconsidering the possible decrease in breakdown amplitude, the Configuration III experiments are also intended to make more clear whether the spanwise features of the roughness array can be correlated to spanwise points of first or last 
breakdown. Such a correlation was expected but not observed in Configuration II. To address this issue, the spanwise array spacing of Configuration III was selected such that it coincided with the fundamental $\Lambda$ vortices, the secondary instability mode that is expected to exist in the boundary layers of interest here. The first objective is to provide clear evidence for the reduction of the breakdown amplitude of unsteady waves in the presence of transient disturbances. The second objective is to investigate any connection between the roughness elements spacing and the breakdown location if it exists.

In this configuration, $U_{\infty}=17.5 \pm 0.9 \mathrm{~m} / \mathrm{s}$ and $f=195 \mathrm{~Hz}$. At typical laboratory conditions this results in $F=64 \pm 7$. The leading edge of the 2D strip with a width of 36 $\mathrm{mm}$ is placed at $x=210 \mathrm{~mm}$ which is slightly upstream of the Branch I point for $F=64$. The strip thickness used is $628 \mu \mathrm{m}$ and this gives $\mathrm{Re}_{\text {ktape }}=328$. The sampling rate is $4160 \mathrm{~Hz}$ and the sample size is 1280 yielding 0.308 seconds per sample. One array of 3D roughness elements of $508 \mu \mathrm{m}$ thickness is used. This array of $\mathrm{Re}_{k}=246$ is placed at $x=160 \mathrm{~mm}$. The spacing between the elements is $\lambda_{\mathrm{k}}=28 \mathrm{~mm}$ and the diameter of the elements is $\lambda_{k} / 2=14 \mathrm{~mm}$. The measurements are taken at 40 spanwise locations with a $3.5 \mathrm{~mm}$ step size for both the baseline (no 3D roughness) and the modulated cases. In this configuration, transient disturbances are introduced to modulate the boundary layer and later they are removed so that receptivity-matched TS experiments can be performed. The shape factor for this configuration is found to be $H=2.58 \pm 0.04$. 


\subsubsection{Spanwise Modulated Basic State - III}

A spanwise array of $\mathrm{Re}_{k}=246$ is used to modulate the baseline case. Variations in $E_{\mathrm{rms}}$ and $A_{s}$ with respect to $x$ is given in Fig. 42. The $E_{\text {rms }}$ data shows significant scatter. However $A_{s}$ is reasonably constant at about $A_{s}=0.09$ across the domain. The displacement thickness variation and the spanwise phase-locked velocity contours are given in Fig. 43 at $R=574(x=300 \mathrm{~mm})$. The high and low speed streaks are clearly seen with strong accelerated regions just coinciding with the edge of the roughness elements.

Figure 44 shows the wall-normal profiles of the unsteady disturbances. This time M-shape modulations cannot be associated with a certain spanwise location. The spanwise modes for both of the modulated cases at $R=663(x=400 \mathrm{~mm})$ are plotted in Fig. 45. The spanwise invariant mode is an order of magnitude larger than the other modes as in the previous configurations. As before, no M-shape modulations are observed in the spanwise invariant mode.

The wall-normal profiles of unsteady disturbances are integrated in the wallnormal direction and averaged across the span to get the amplitudes. Figure 46 shows the growth of unsteady amplitudes in the streamwise direction. A second set of experiments is performed without roughness elements and the unsteady amplitudes at $R=683$ $(x=425 \mathrm{~mm})$ are matched with the modulated case by increasing the sound amplitude. The location is chosen as upstream as possible to have enough length downstream to make the growth rate reduction more clear. The amplitudes of the unmodulated case are also plotted in Fig. 46 and it can be seen that the growth rate of the unsteady disturbances is significantly reduced in the presence of transient disturbances. 
The breakdown pattern of modulated unsteady waves is investigated by plotting intermittency variations of several $x$ locations with respect to $z$ as shown in Fig. 47. It can be seen that breakdown locations are perfectly correlated with the roughness array spacing. The largest intermittencies are observed at exactly the sides of the roughness elements (i.e., $z / \lambda_{k}= \pm 1 / 4$ ) where there is a strong accelerated region as seen in Fig. 43. Moreover, the breakdown locations are aligned with respect to each other in the span for different streamwise locations. This pattern is suggestive of Fig. 6a and it is suspected that this is fundamental breakdown through K-type $\Lambda$ vortices.

The streamwise points of the initial breakdown are $x=525 \mathrm{~mm}(R=759)$ and $x=675 \mathrm{~mm}(R=861)$ for the modulated and unmodulated cases, respectively. The spanwise-averaged unsteady disturbance amplitudes at these locations are $A=0.06$ and $A=0.16$, respectively. The breakdown amplitude of unsteady disturbances in the modulated case is only slightly more than one third of the amplitude in the nonmodulated case.

Table 5 gives the breakdown location and unsteady disturbance amplitudes just prior to breakdown for Configuration III cases. The breakdown location moves upstream even though the growth rate is reduced because of significant reduction in the breakdown amplitude. 


\begin{tabular}{|c|c|c|}
\hline & Breakdown Location & Breakdown Amplitude \\
\hline $\operatorname{Re}_{k}=246$ Modulated Case & $x=525 \mathrm{~mm}$ & 0.06 \\
\hline $\begin{array}{c}\text { Non-modulated case amplitude- } \\
\text { matched to } \mathrm{Re}_{k}=246\end{array}$ & $x=675 \mathrm{~mm}$ & 0.16 \\
\hline
\end{tabular}

Table 5: Breakdown locations and unsteady disturbance amplitudes at breakdown for Configuration III cases

As a summary of the results of this configuration, it is again verified that the growth rate of unsteady disturbances is reduced and can be strongly reduced by large-amplitude transient disturbances. Similar to the earlier findings, the spanwise decomposition of these disturbance profiles revealed that there are no M-shape modulations in the spanwise invariant mode similar. Most importantly, it was found that the breakdown amplitude reduction is very significant compared to growth rate reduction and this results in sooner transition when transient disturbances are introduced. Additionally, the edge of the roughness elements where there is strong accelerated region is found to be most prone to breakdown. 


\section{Chapter 4}

\section{SUMMARY AND CONCLUSIONS}

Numerical simulations (Cossu and Brandt 2002, 2004) (CB) have shown that stationary, spanwise periodic transient optimal disturbances are capable of suppressing the growth of Tollmien-Schlichting (TS) waves in boundary layers. Previous experiments that could have verified this effect (e.g., Corke et al. 1986; Boiko et al. 1994) were conducted before the theory was developed and did not directly address CB's predictions. Moreover, those experiments have tended to show that roughness (and perhaps the transient growth it initiates) leads to accelerated TS-wave breakdown. A more recent study by Shelley (2004) was able to verify some of the CB predictions but not the most significant regarding growth rate reductions. The study presented here attempts to make an unambiguous determination of whether TS wave growth can indeed be suppressed by transient disturbances and, if this suppression is physically realizable, whether the growth-rate reduction can delay breakdown to turbulence in TS-dominated transition. To accomplish this, three experimental configurations are designed that progressively achieve these objectives. In all three, controlled TS waves are generated using a combination of acoustic forcing and a two-dimensional roughness strip and transient disturbances are created using a spanwise array of cylindrical roughness elements.

The first set of experiments revealed M-shape modulations in the wall-normal profiles of the unsteady disturbances as predicted by CB. CB reported M-shape modulations in the spanwise invariant mode of the unsteady disturbances. However, this 
was not observed in the experiments. Instead, M-shape modulations were found to arise from the fundamental and harmonic spanwise modes. There are several potential explanations of this behavior that are related to the fact that the numerical simulations do not consider receptivity details and deal with optimal disturbance but the experiments necessarily consider all receptivity effects and sub-optimal disturbances. In addition to modulating the disturbance profiles, the transient disturbances were also observed to slightly reduce the growth rate of the unsteady disturbances. The small reduction was most likely related to low transient disturbance amplitudes that were produced in this first experimental configuration. The most dramatic and surprising effect that appeared in Configuration I was that the receptivity of unsteady waves in the presence of transient disturbances was significantly reduced relative to the baseline configuration without 3D roughness. The reduction of the unsteady disturbances initial amplitudes became pronounced as the transient disturbance amplitudes were increased. With regards to the transition delay, both the reduced growth rate and the reduced receptivity led to lower unsteady wave amplitudes at given streamwise location which did result in transition delay. However, the receptivity reduction is a different mechanism from that considered by $\mathrm{CB}$ and it is not clear whether this reduction is unique to this configuration or is a more universal effect. Further experiments are necessary to address the receptivity of unsteady waves in detail.

The second set of experiments performed with larger transient disturbances revealed M-shape modulations in the wall-normal profiles of the unsteady disturbances similar to the first setup and unambiguously verified that the growth rate of unsteady waves are reduced by transient disturbances. As in Configuration I, M-shape modulations 
are not observed in the spanwise invariant mode, only in the fundamental and harmonic modes.

Measurements of turbulent intermittency across the experimental domain and comparing the breakdown amplitudes and locations of unsteady waves by matching the reduced receptivity hinted an interesting result. It was found that the breakdown amplitudes for the modulated unsteady waves were slightly lower than their receptivitymatched non-modulated counterparts. With regards to transition delay, this suggests that even though the growth rate of unsteady waves is reduced, transition delay may not be achieved in terms of breakdown location. However, the Configuration II experiments did not make it possible to compare the relative effect of these mechanisms transition location.

The third experimental configuration was specifically designed to address the competing mechanisms of growth rate reduction and breakdown amplitude reduction. As for Configuration II, Configuration III makes use of initial-amplitude matching. In this case, M-shape modulations and growth rate reduction of unsteady waves are observed as expected. One important result of these receptivity matched experiments is that the breakdown amplitude is significantly smaller in the modulated cases than non-modulated cases and is found to be more significant than the growth rate reduction. This has the effect of moving the onset of transition upstream, an effect that is (usually) opposite of what is desired. There is some evidence that breakdown may occur via fundamental, Ktype $\Lambda$ vortices as is expected in this configuration. However, this is not conclusively established by the present results. 
Even though the growth rate reduction predicted by CB sparked the notion that introducing controlled transient disturbances may be implemented as a transition delay mechanism, the results of this work suggests that there are other, sometimes counterbalancing effects that make implementing this control strategy difficult. Further studies are necessary to make a clear conclusion about the possibility of using transient growth as a means of controlling transition. First, the underlying mechanism associated with the reduced receptivity of unsteady waves in the presence of transient disturbances needs to be addressed. Second, the mechanism underlying reduced breakdown amplitudes needs to be resolved. 


\section{Appendix A}

\section{FIGURES}

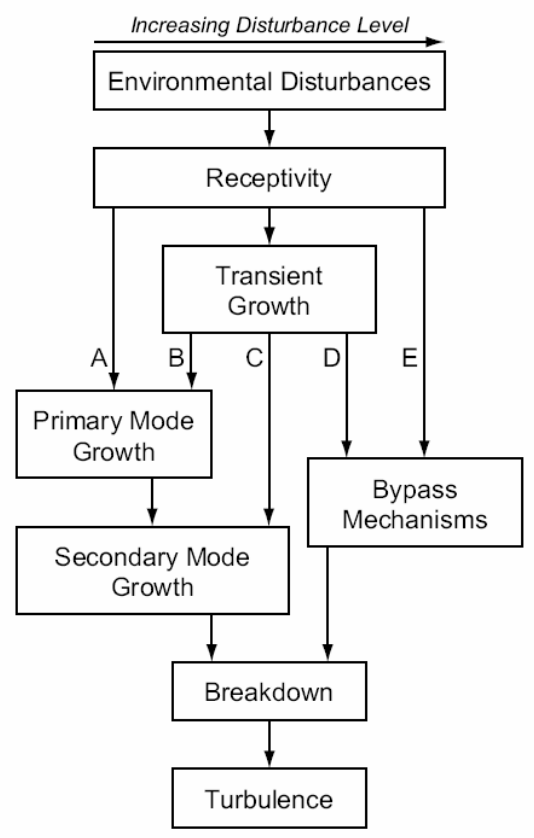

Figure 1: Transition roadmap, adapted from Morkovin et al. (1994) 


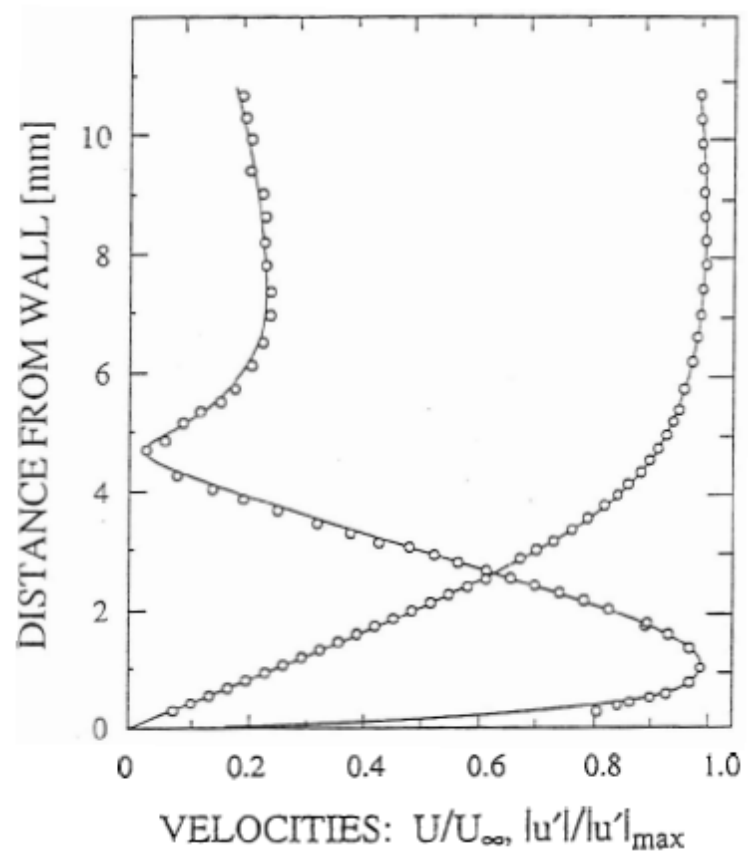

Figure 2: Wall normal Blasius mean flow and disturbance profiles of TS waves, adapted from Saric (1990)

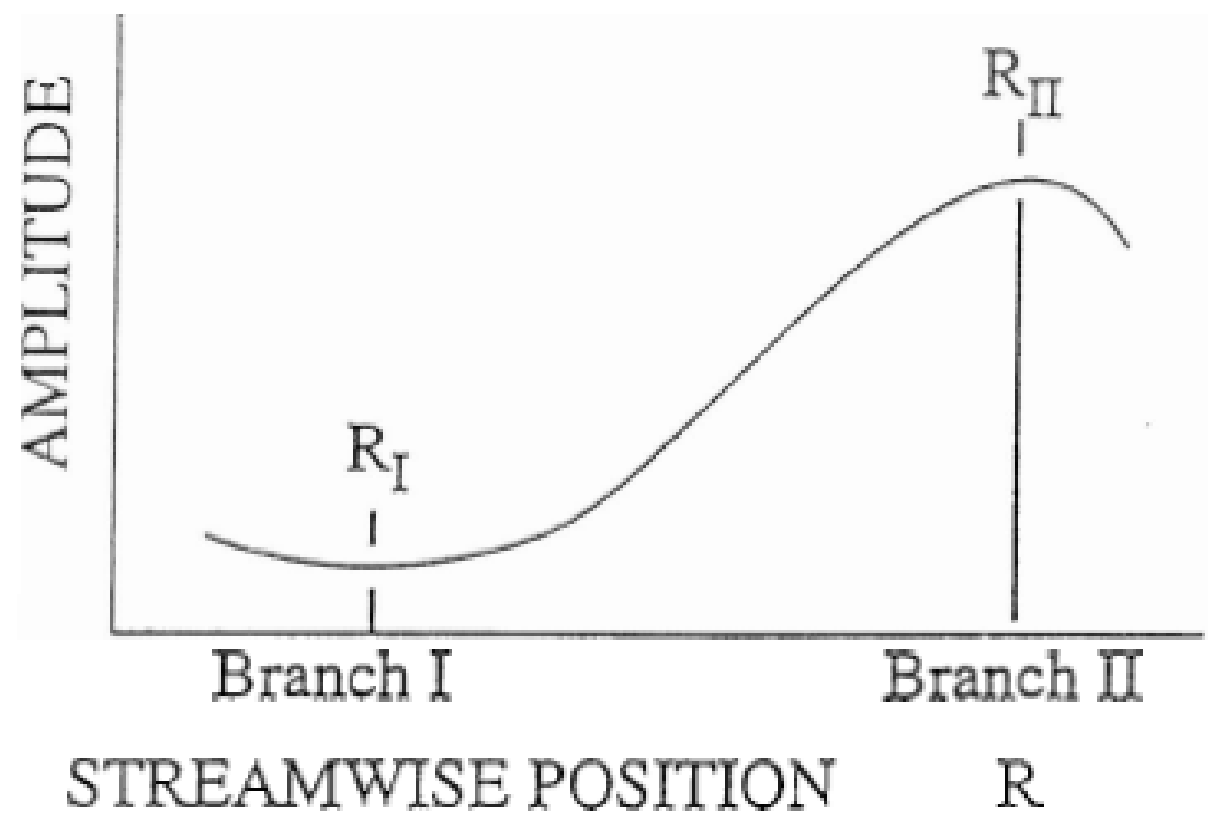

Figure 3: Schematic of TS amplitude change in the streamwise direction 

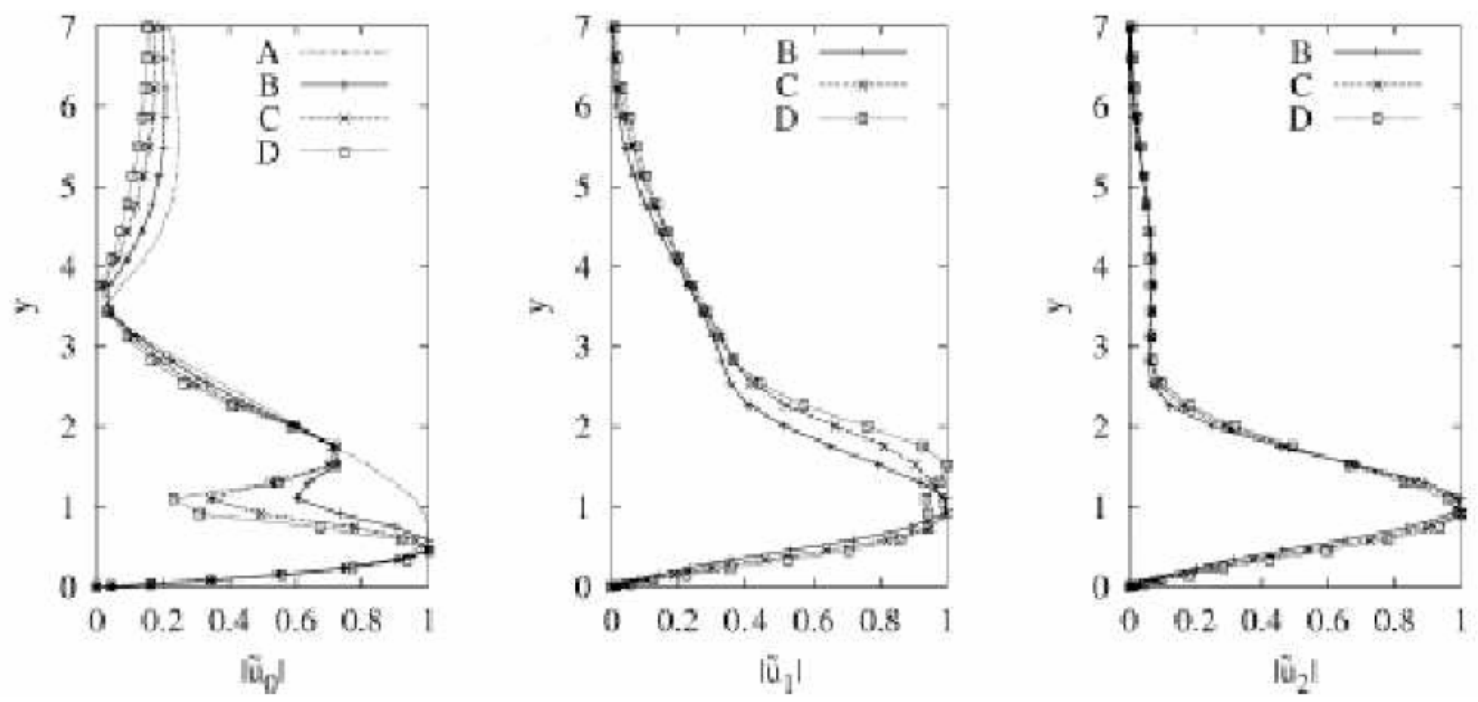

Figure 4: Disturbance profiles computed by Cossu and Brandt (2002, 2004), Cases B to D represent increasing transient growth amplitude

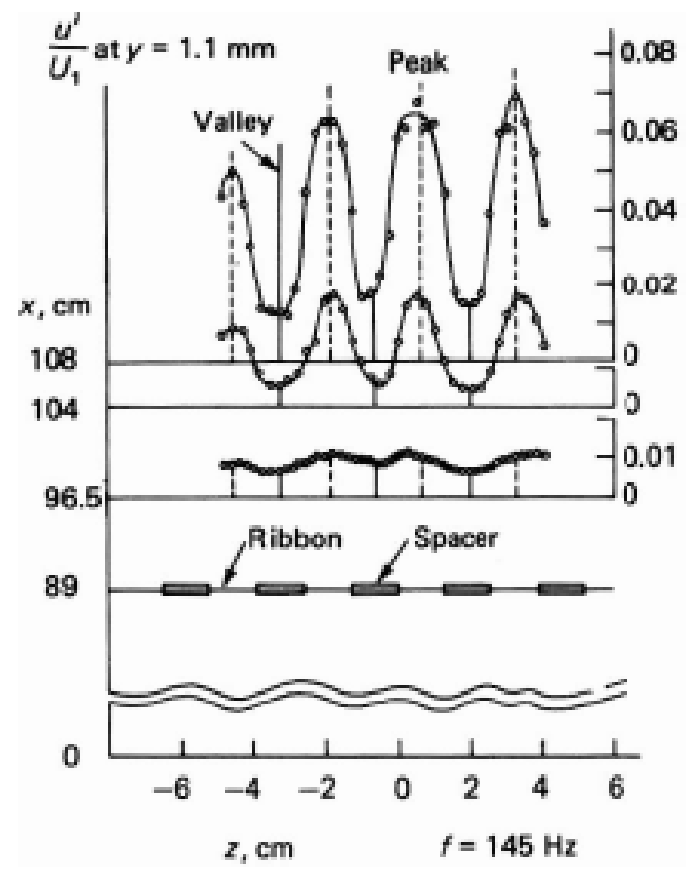

Figure 5: Development of spanwise peaks and valleys in the TS wave amplitudes, adapted from Klebanoff et al. (1962) 


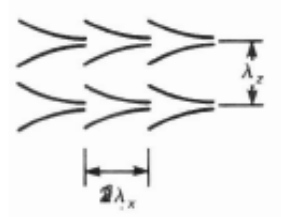

(a)

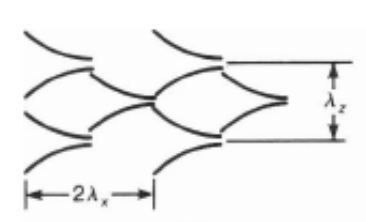

(b)

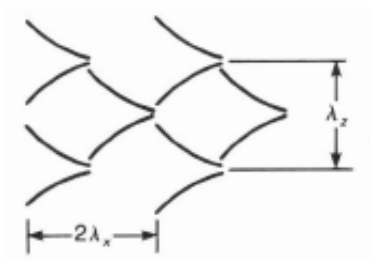

(c)

c)
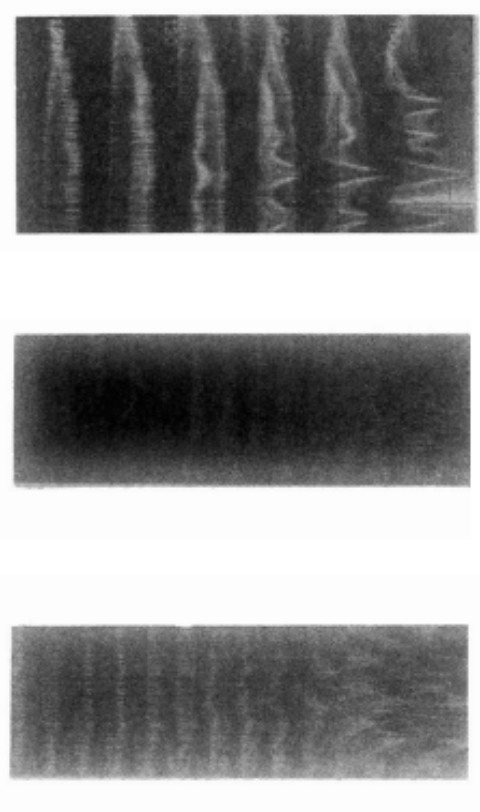

$$
\text { . }
$$

Figure 6: Patterns of $\Lambda$-type vortices: a) K-type b) C-type c) H-type, after Saric and Thomas (1984)
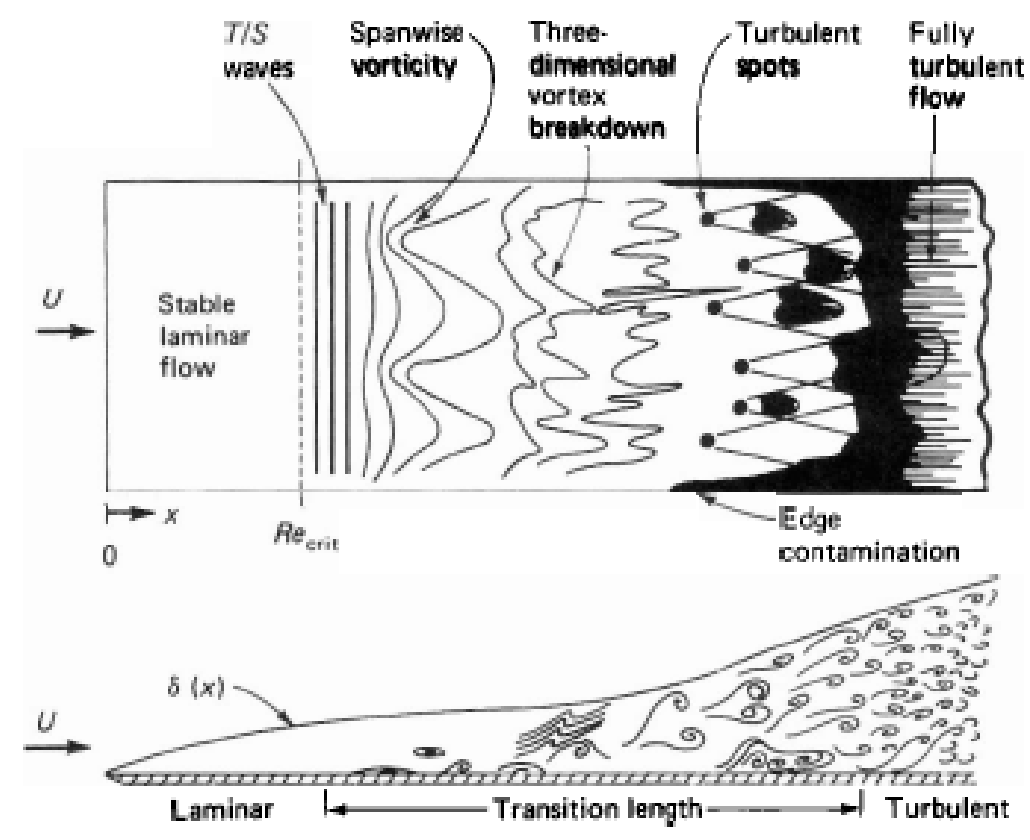

Figure 7: Sketch of boundary layer transition process, after White (1991) 


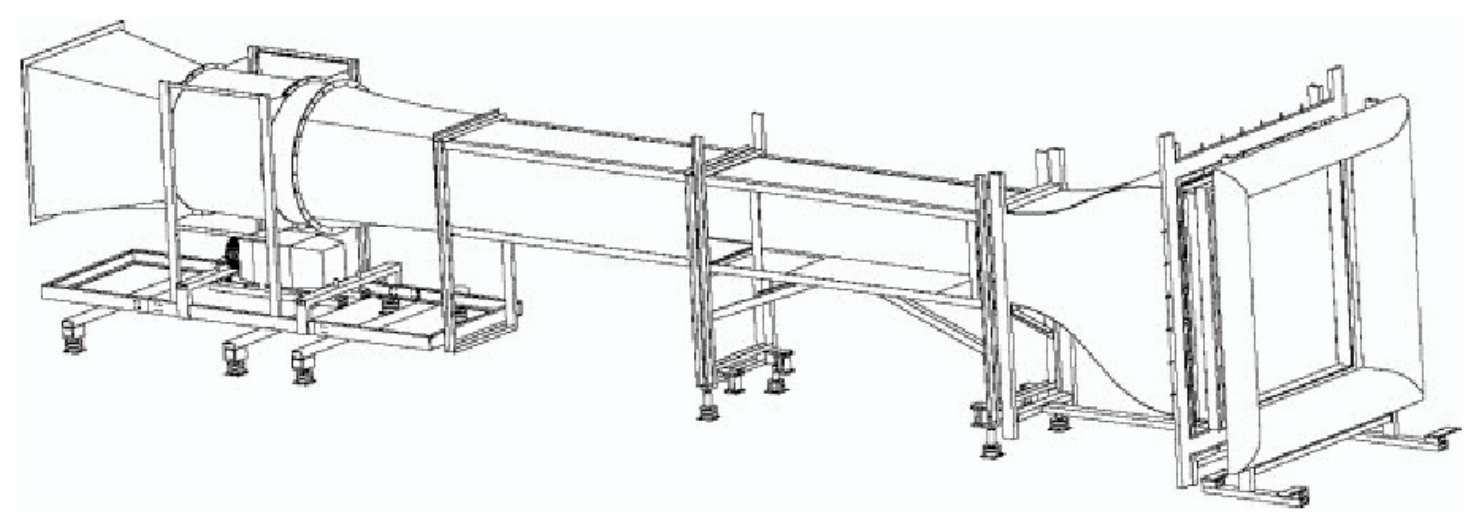

(a)

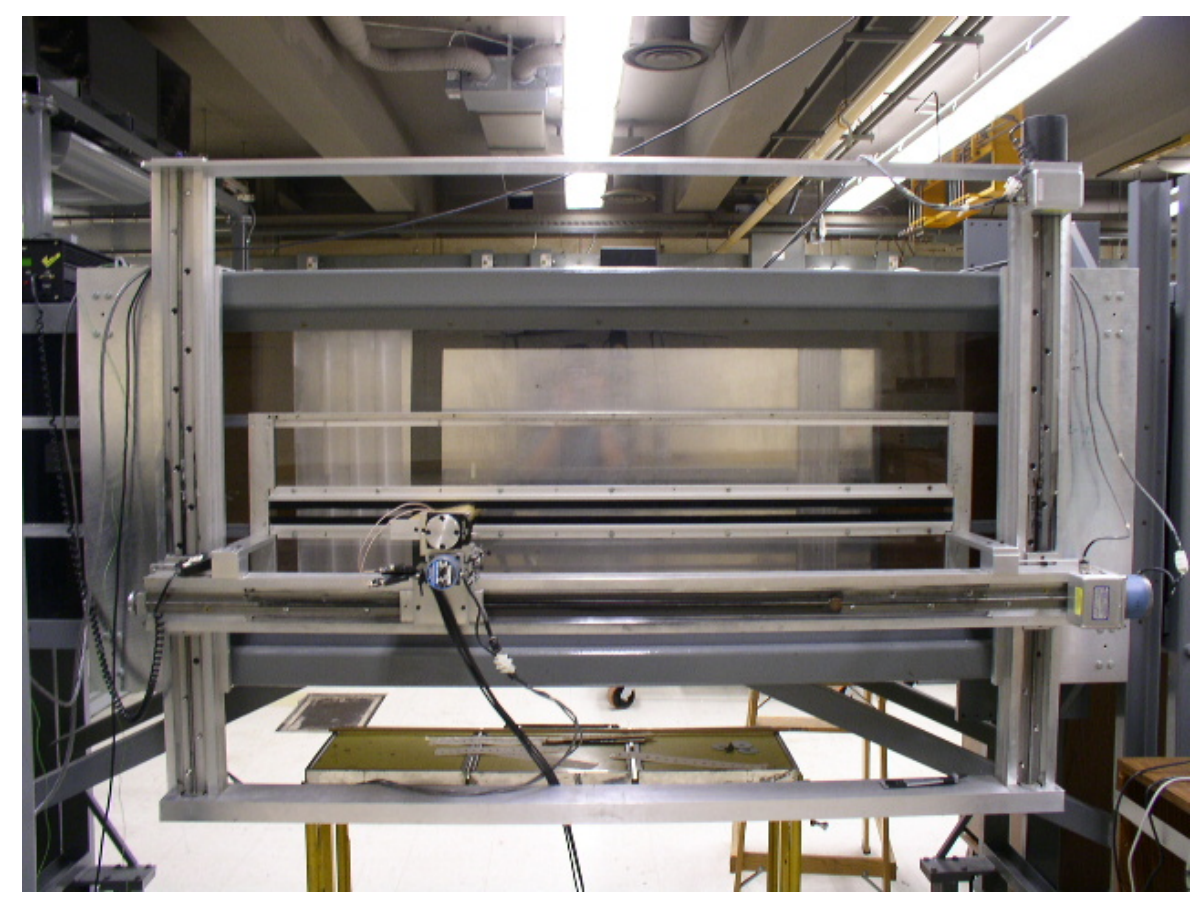

(b)

Figure 8: (a) Sketch of Case wind tunnel. This sketch does not include the hotwire traverse system and only includes one turbulence screen (b) Photograph of the test section 


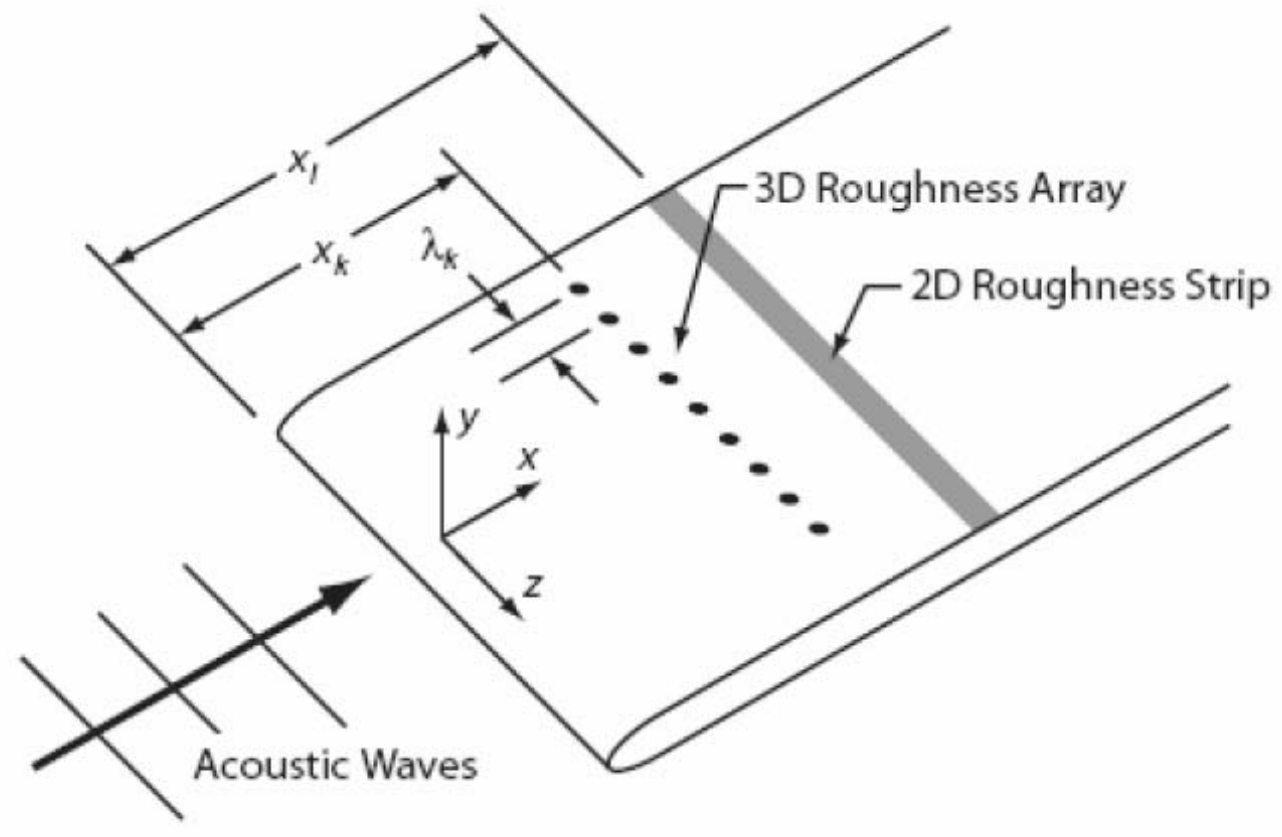

Figure 9: Schematic illustration of the experimental configuration

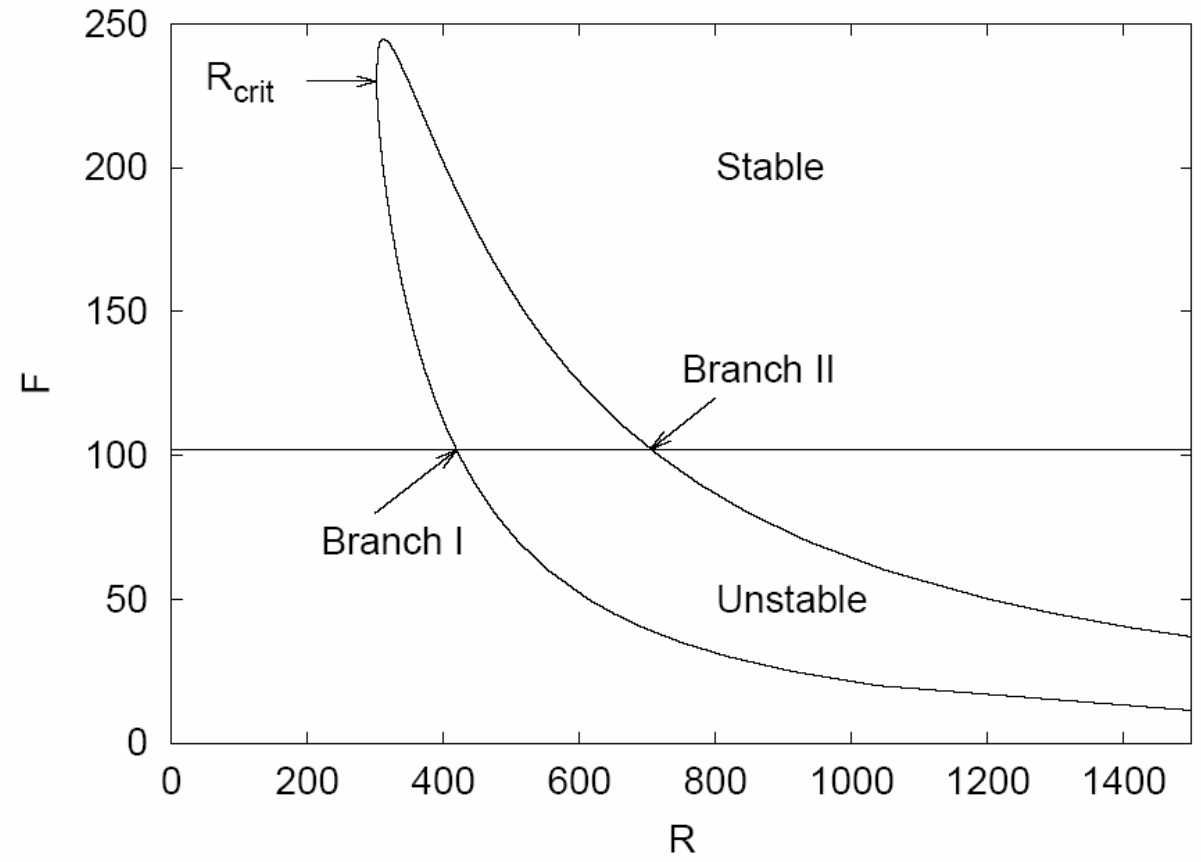

Figure 10: Sketch of TS wave neutral stability curve 


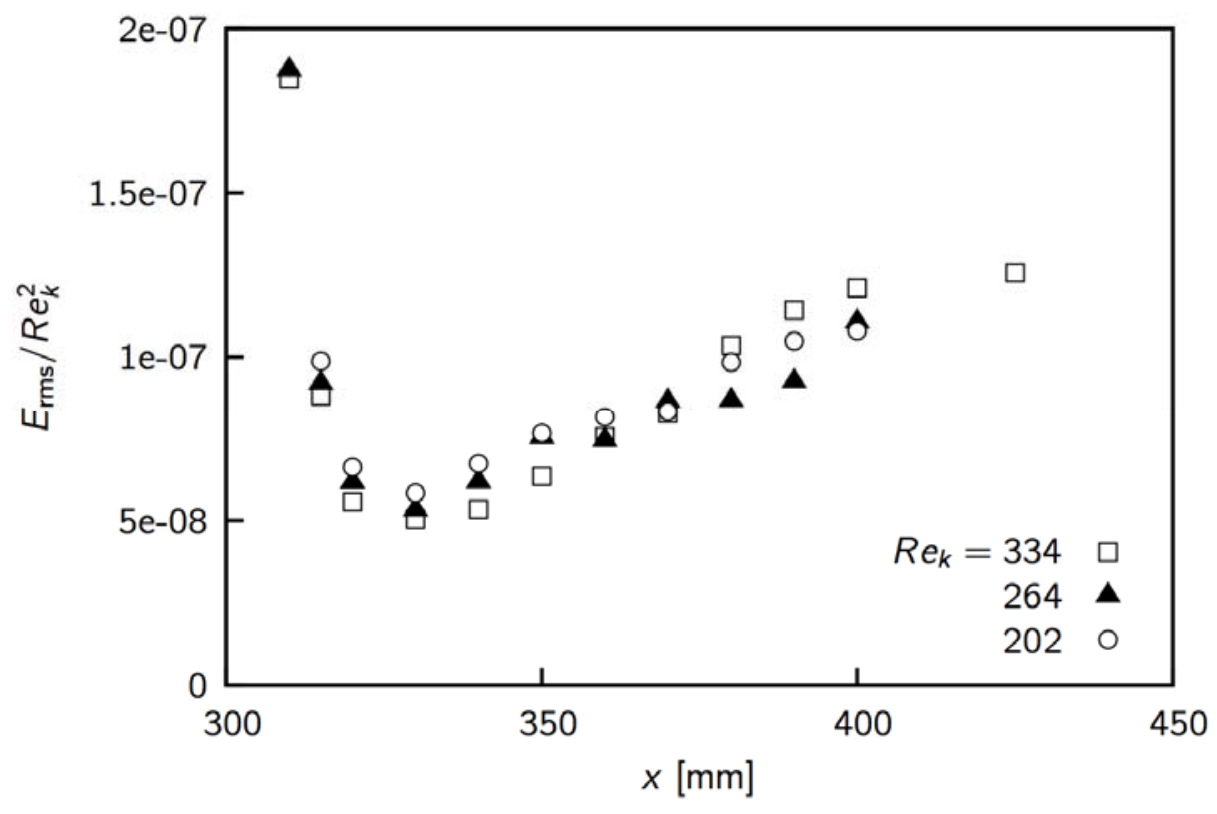

Figure 11: Typical total stationary disturbance energy variation, after Ergin and White (2005) 


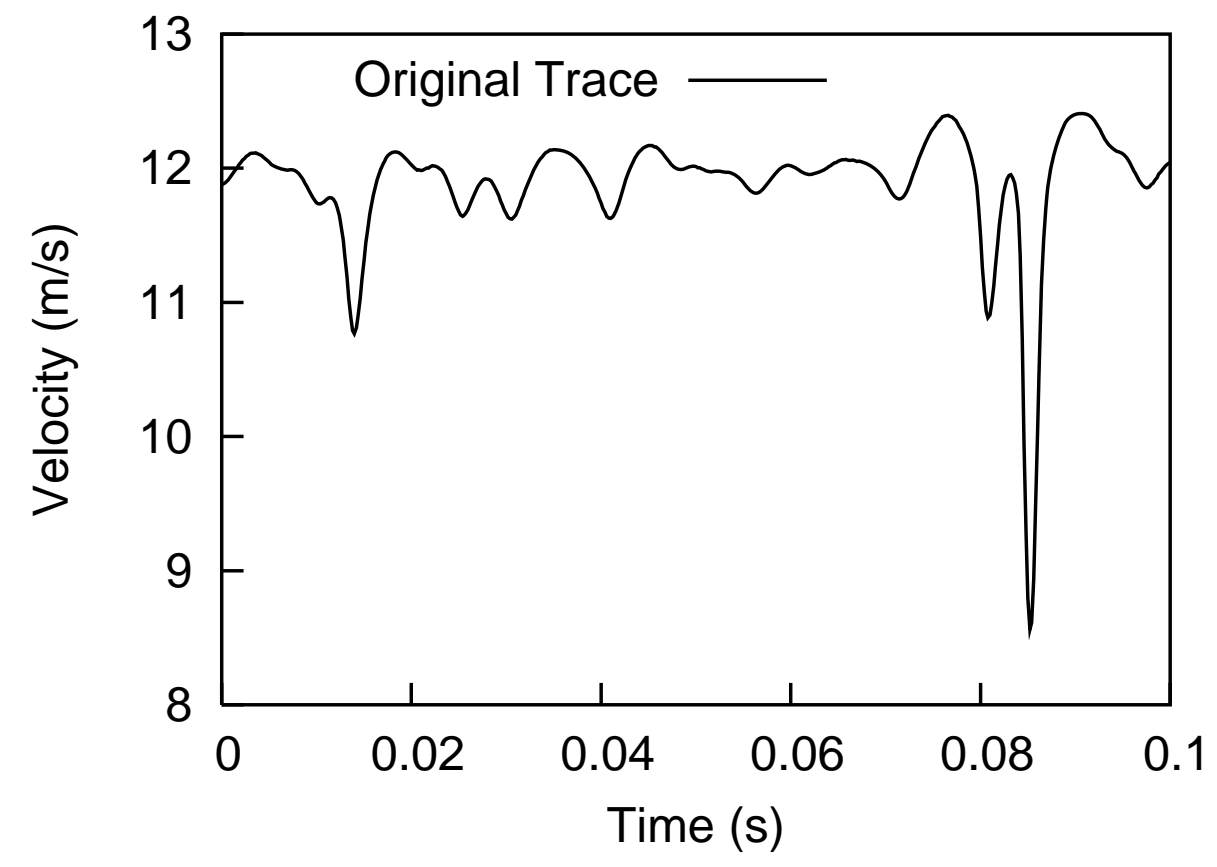

(a) Original velocity trace

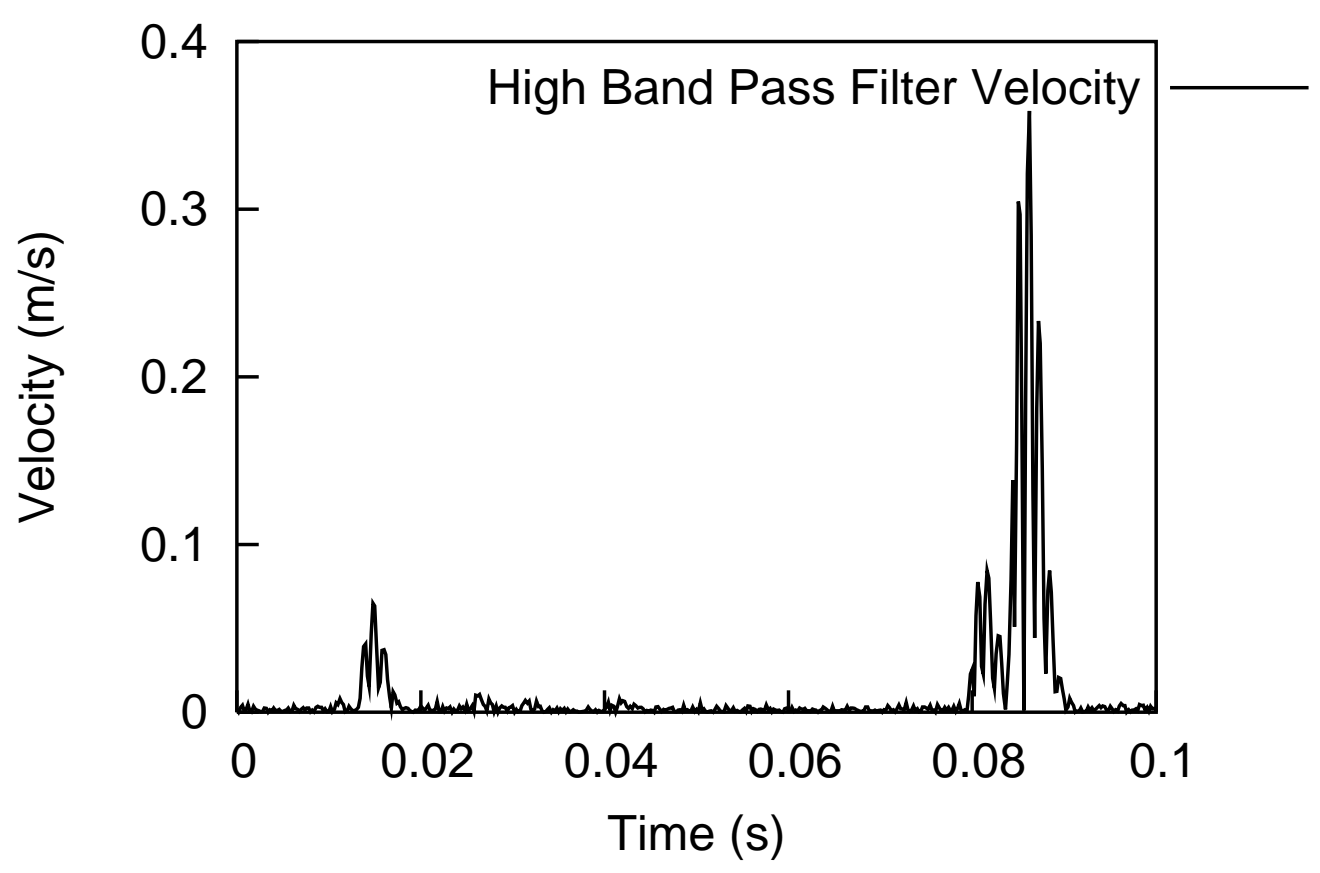

(b) High band pass filtered velocity 


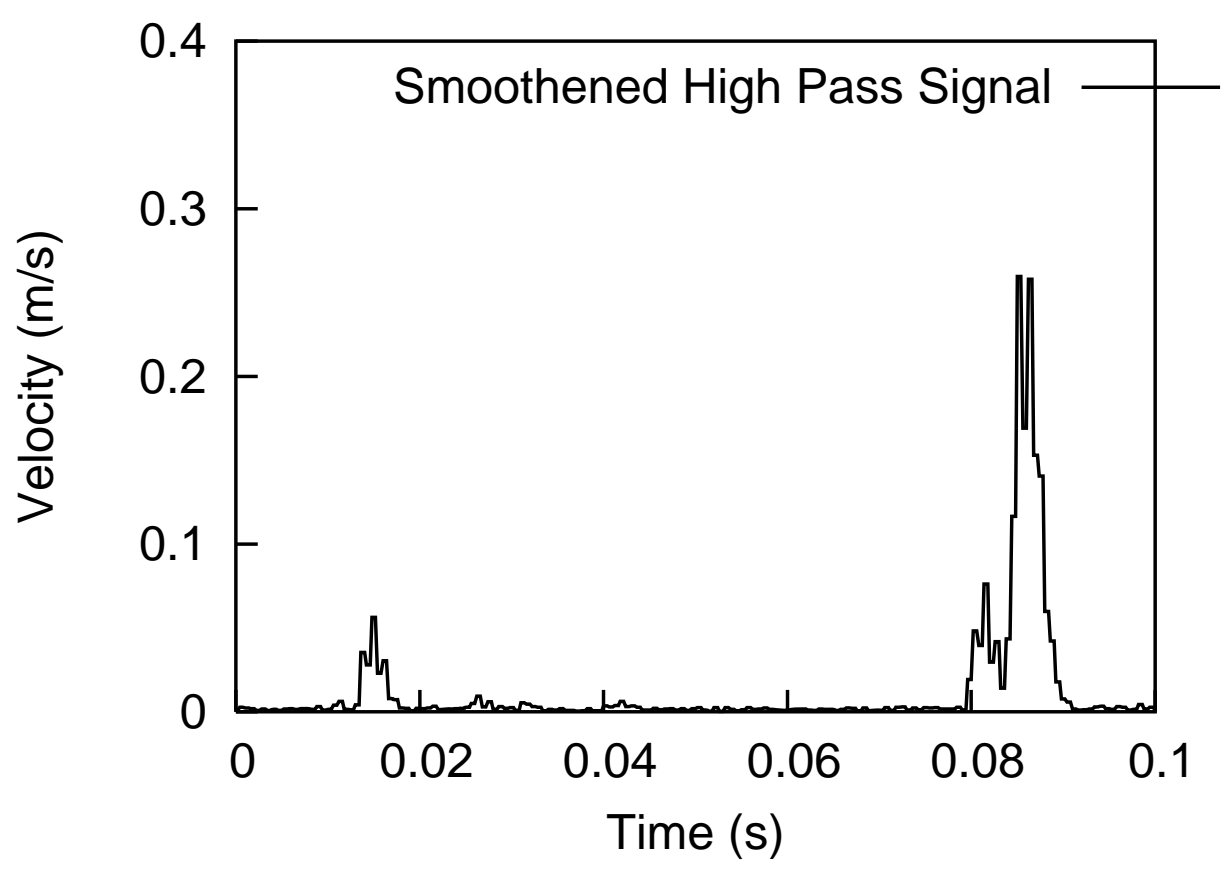

(c) Short-term averaged high band pass signal

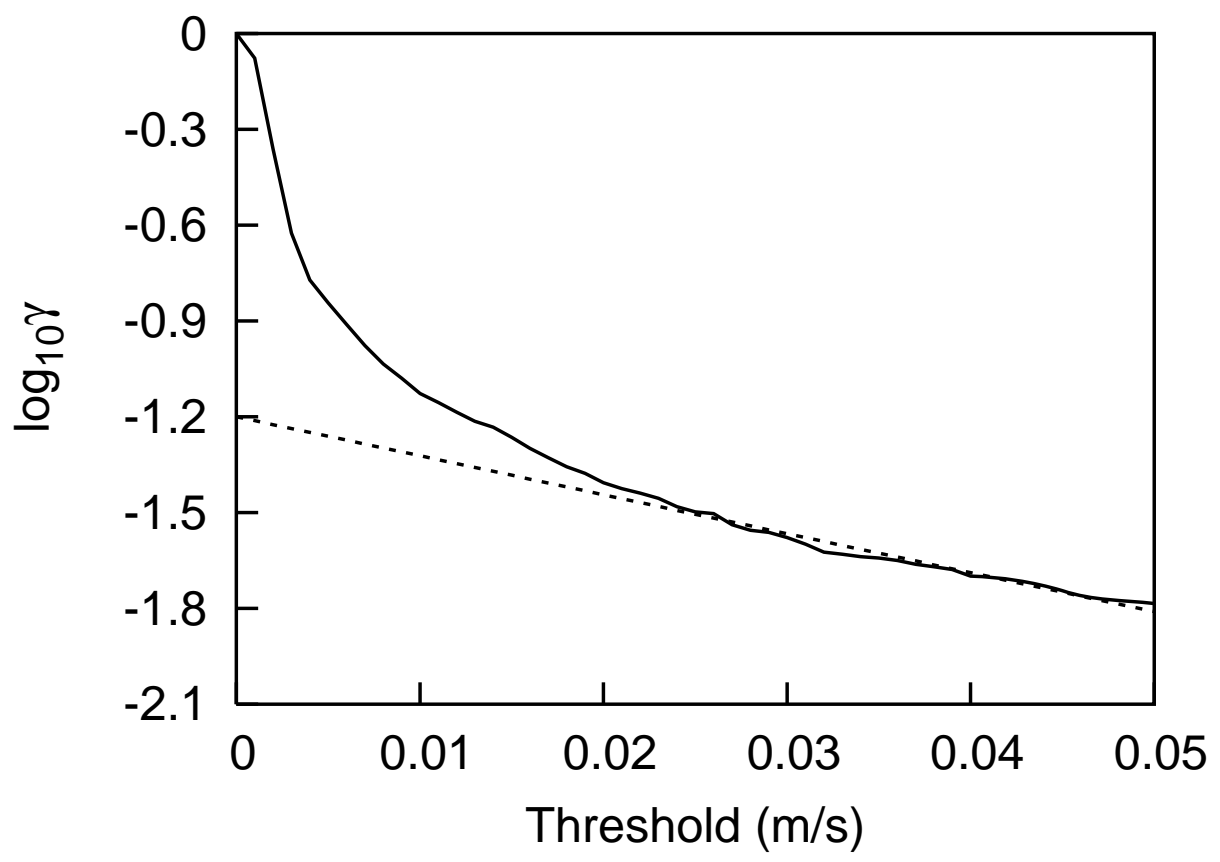

(d) Threshold variation and selection procedure 


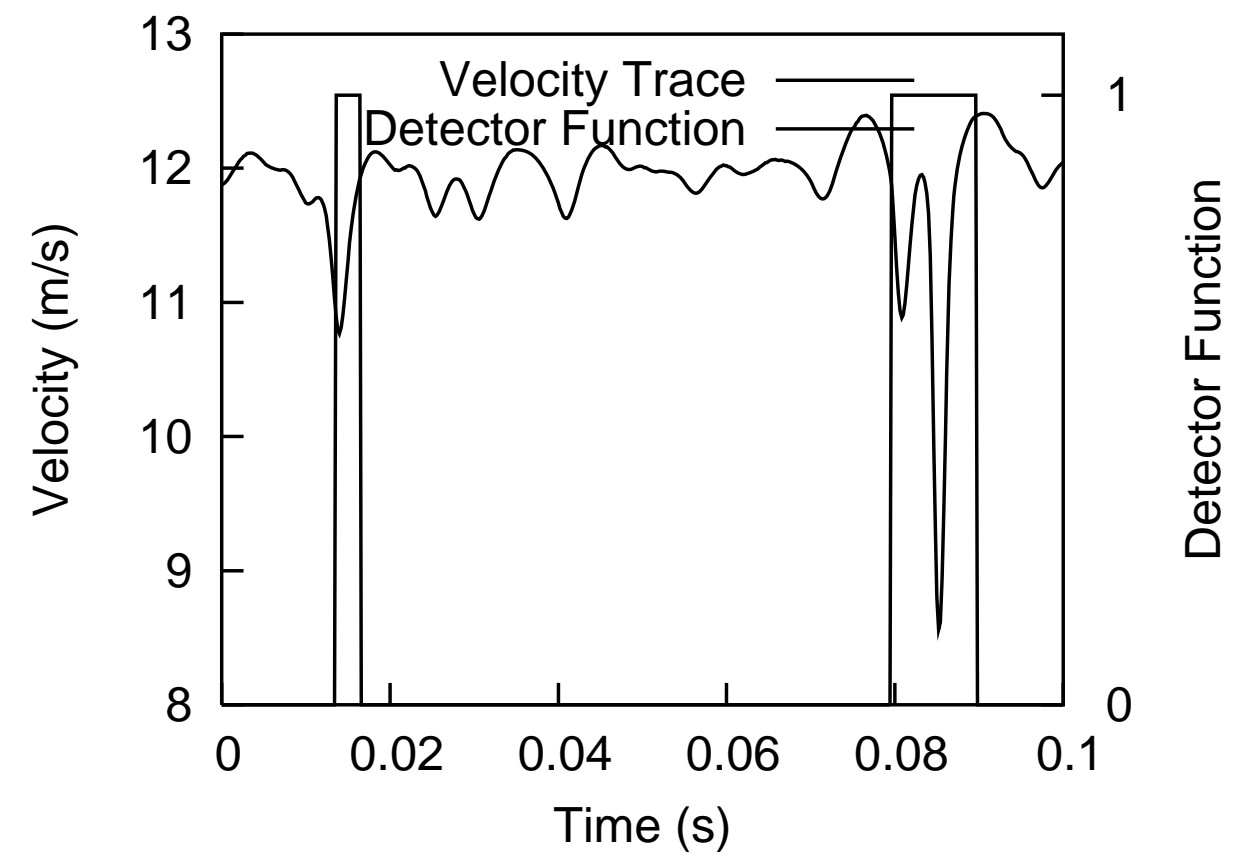

(e) Detector function superposed on velocity trace

Figure 11: Intermittency estimation procedure. For this particular sample, intermittency is estimated to be $6.2 \%$. 

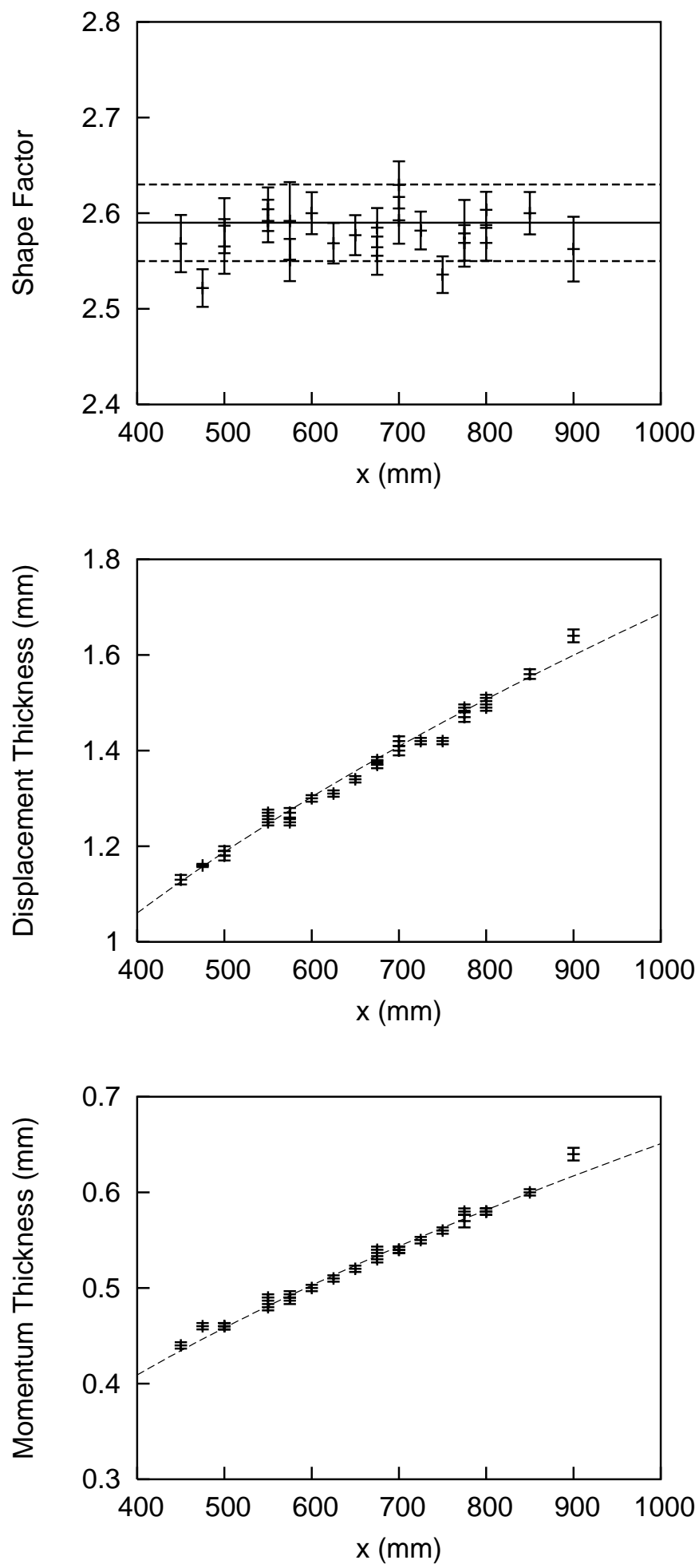

Figure 12: Conf. I - Displacement and Momentum Thicknesses, Shape Factor 


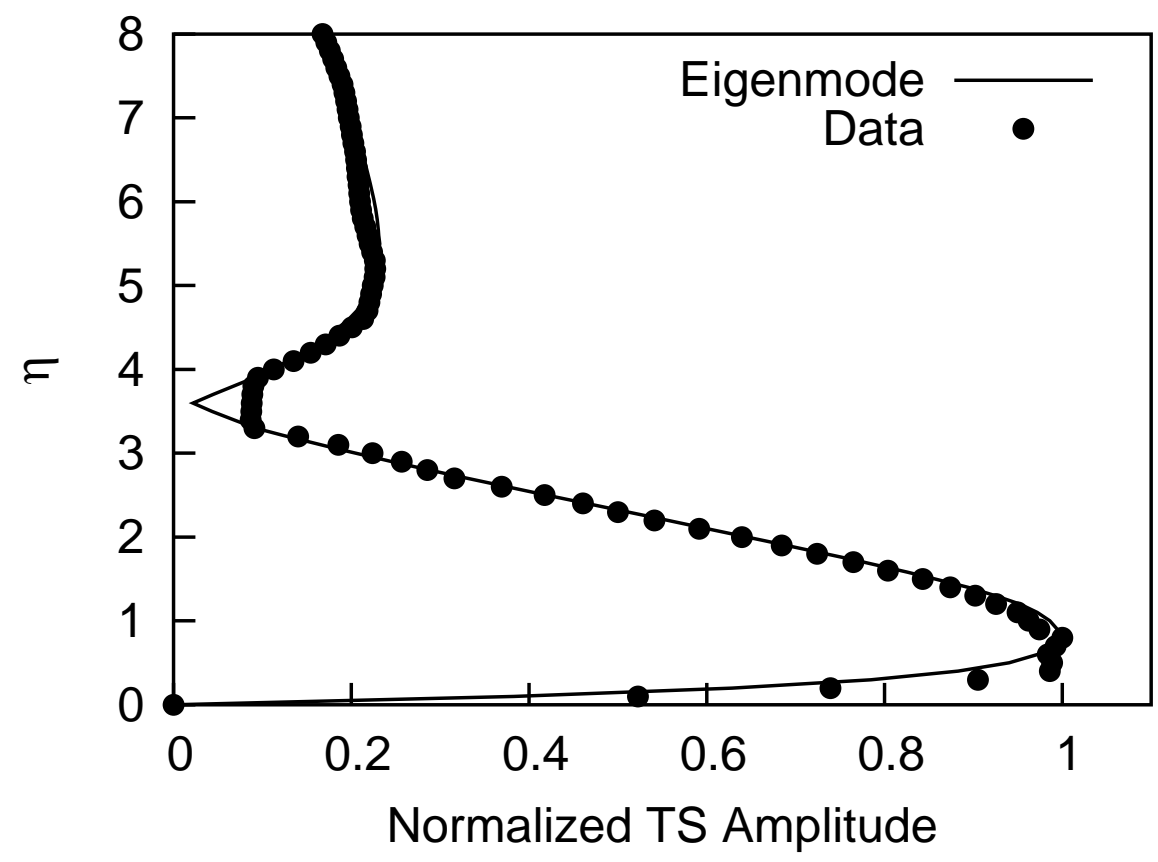

Figure 13: Conf. I - Spanwise mean TS wave profile at $\mathrm{R}=845$

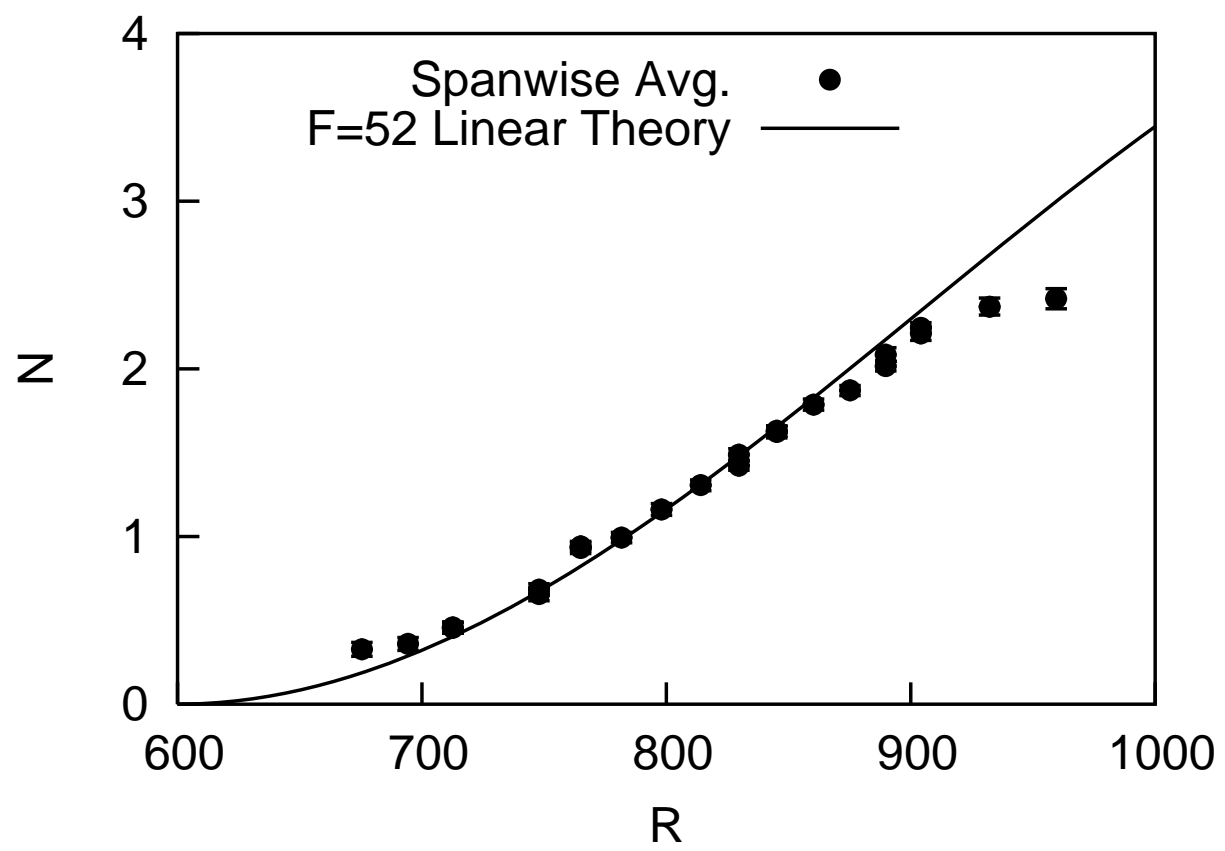

Figure 14: Conf. I - N-factor curve 

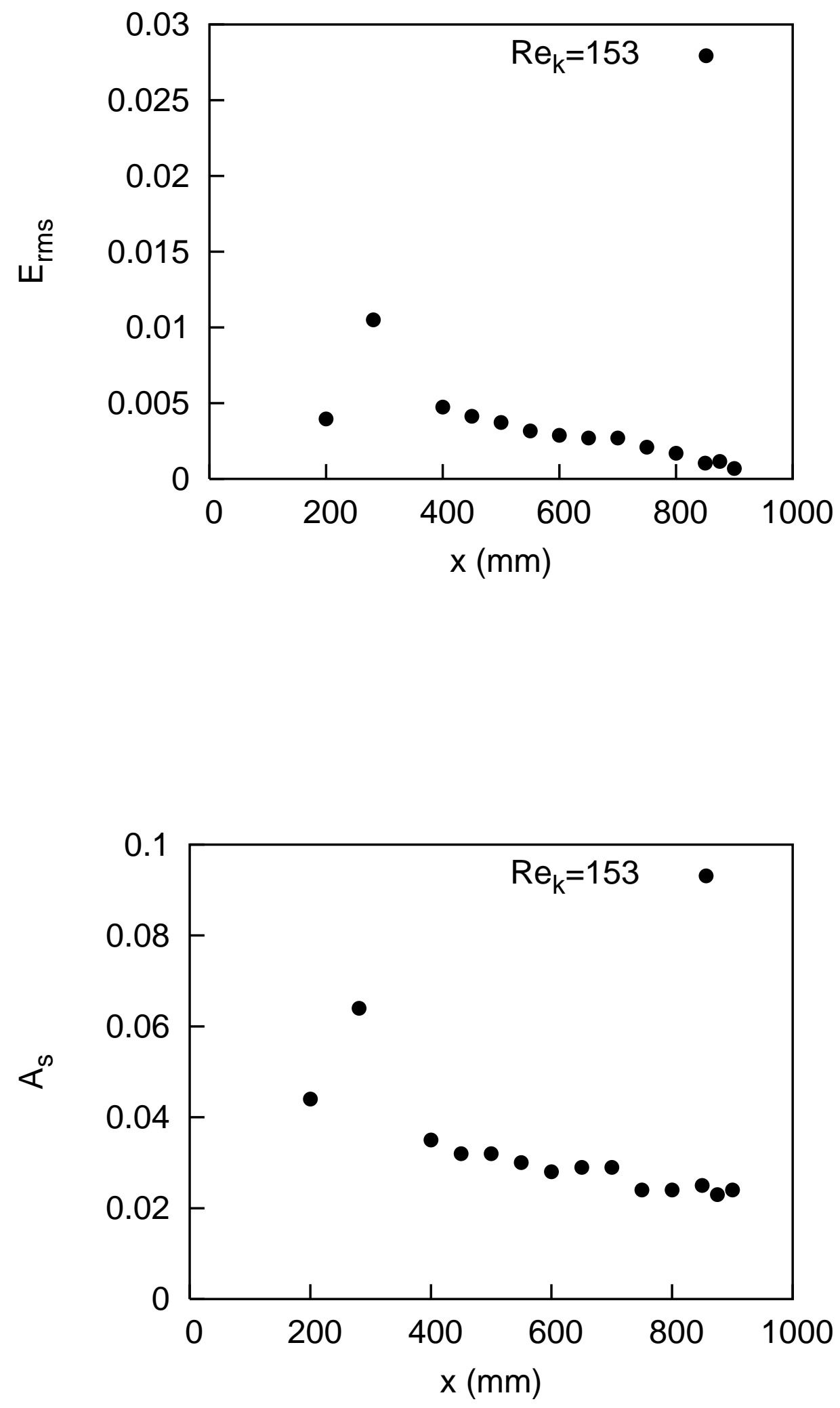

Figure 15: Conf. $\mathrm{I}-\mathrm{E}_{\mathrm{rms}}$ and $\mathrm{A}_{\mathrm{s}}$ variation for $\mathrm{Re}_{\mathrm{k}}=153$ 

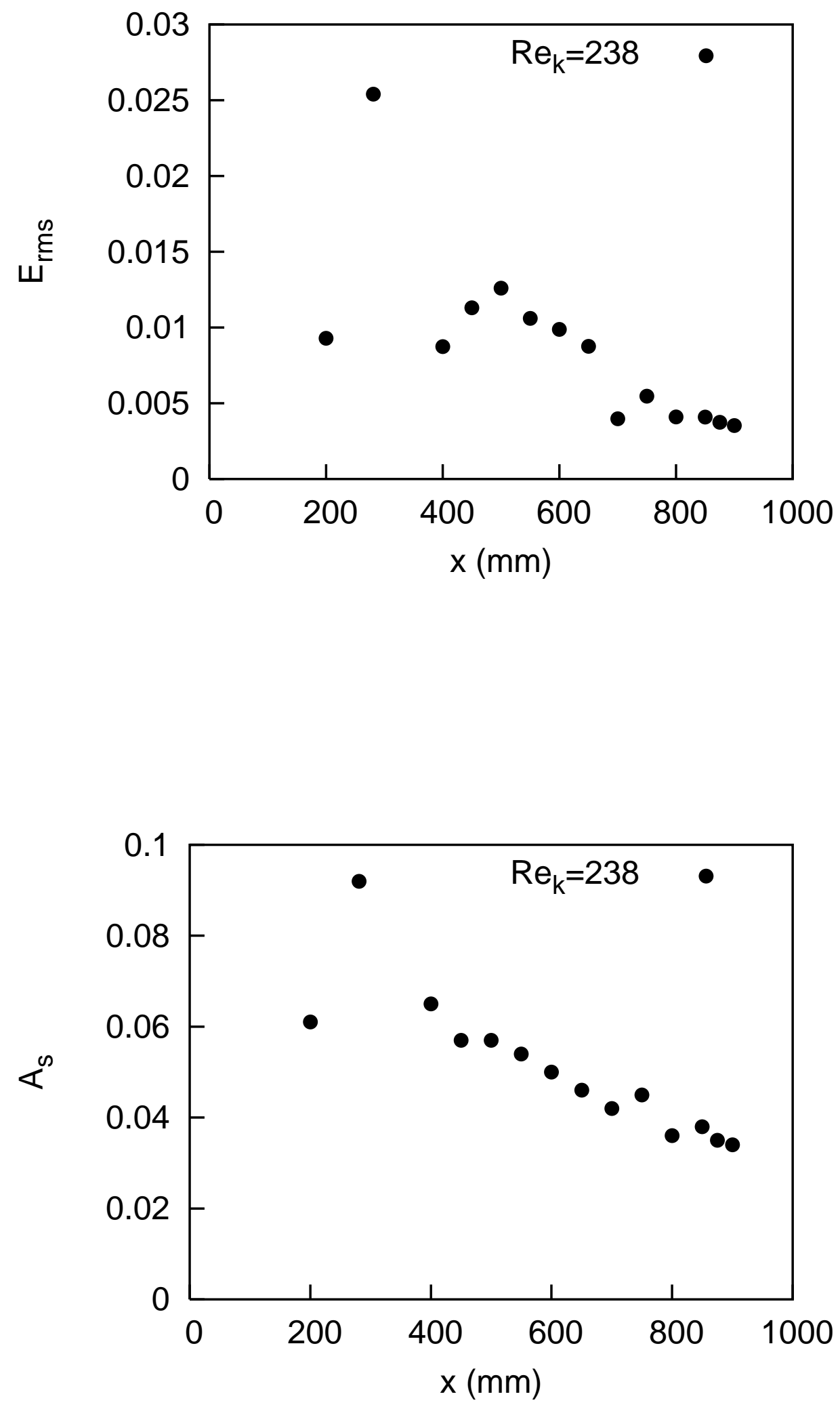

Figure 16: Conf. $\mathrm{I}-\mathrm{E}_{\mathrm{rms}}$ and $\mathrm{A}_{\mathrm{s}}$ variation for $\mathrm{Re}_{\mathrm{k}}=238$ 

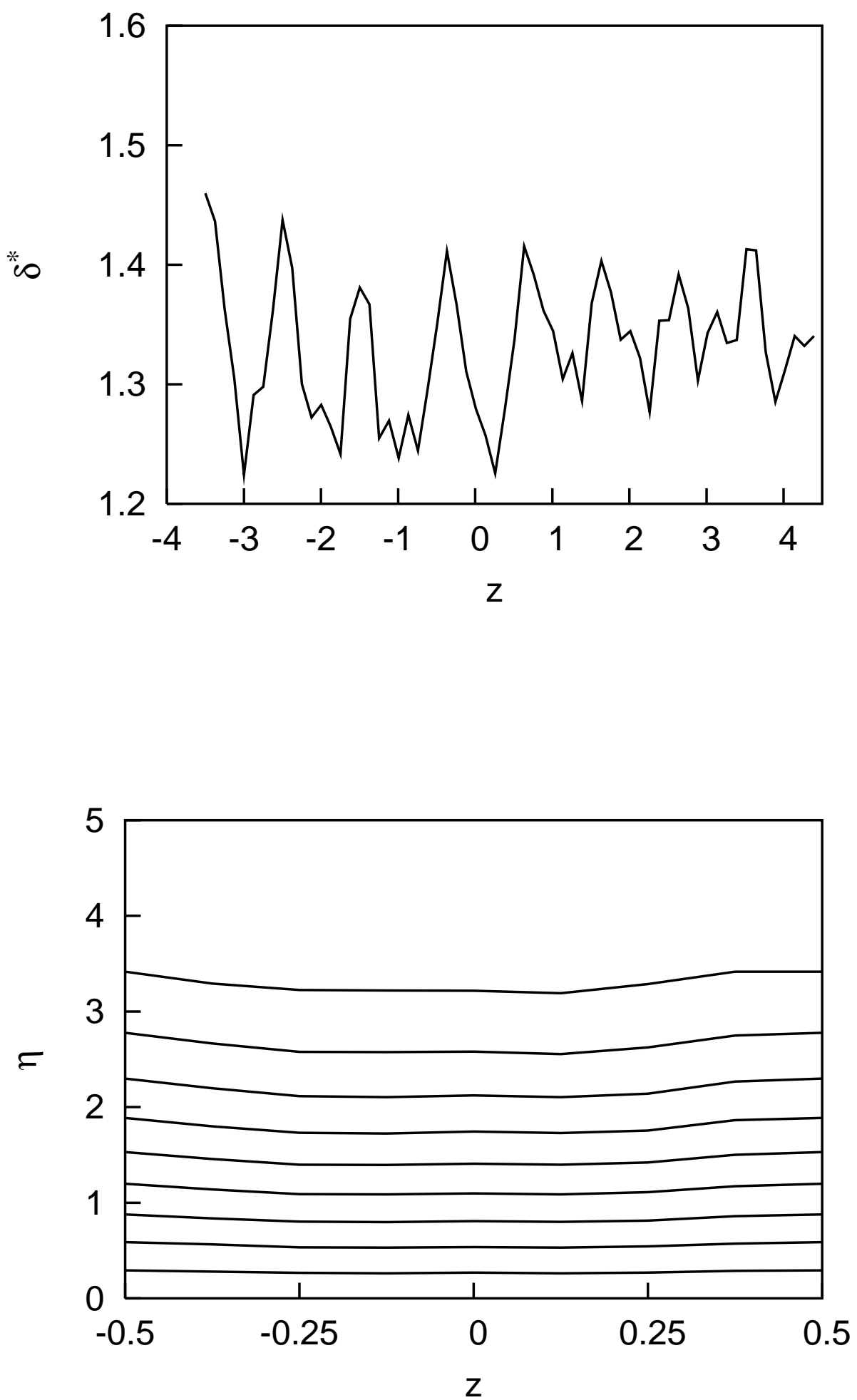

Figure 17: Conf. I - Displacement thickness variation and the velocity contours for $\mathrm{Re}_{\mathrm{k}}=153$ at $\mathrm{R}=814$ 

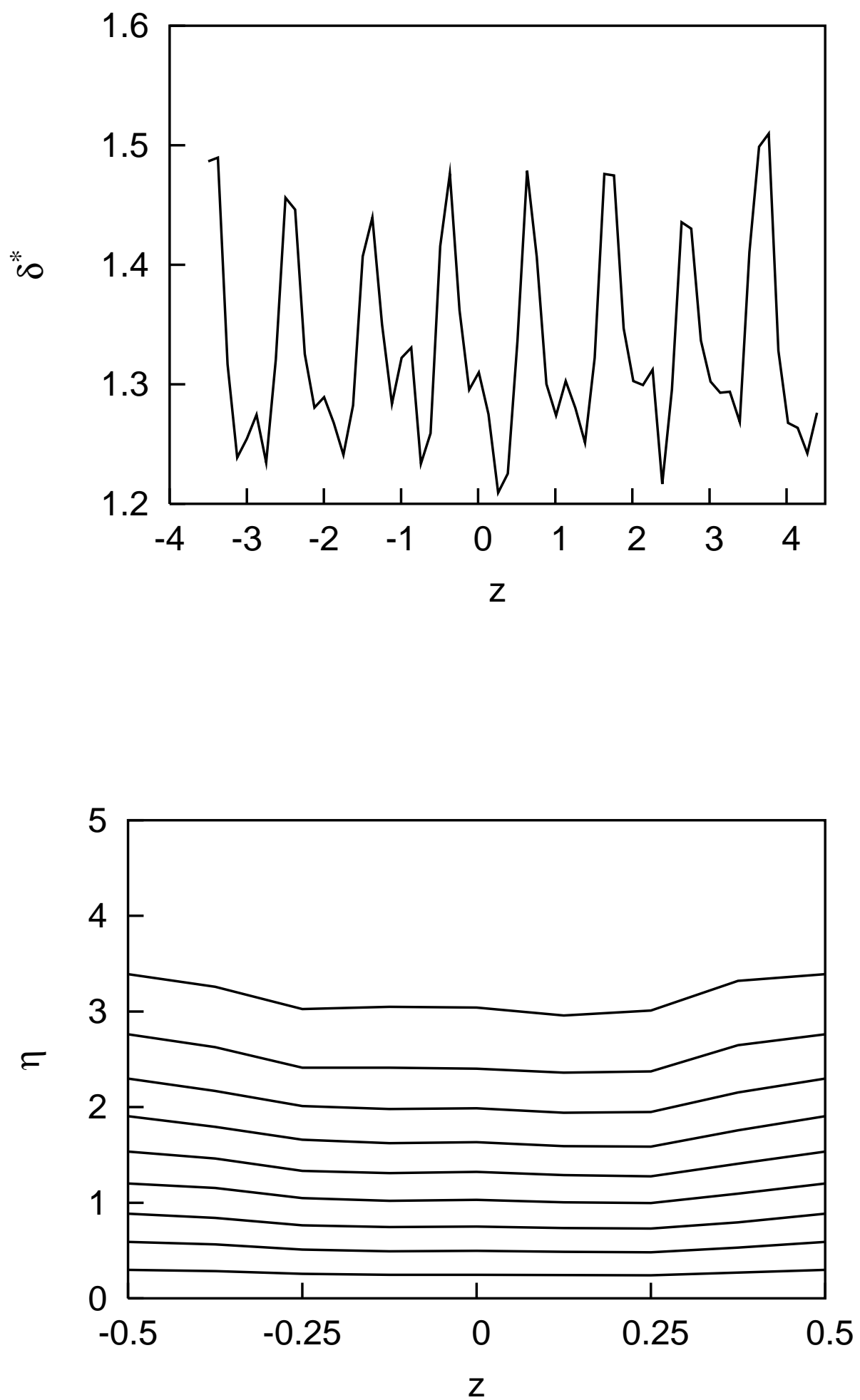

Figure 18: Conf. I - Displacement thickness variation and the velocity contours for $\mathrm{Re}_{\mathrm{k}}=238$ at $\mathrm{R}=814$ 

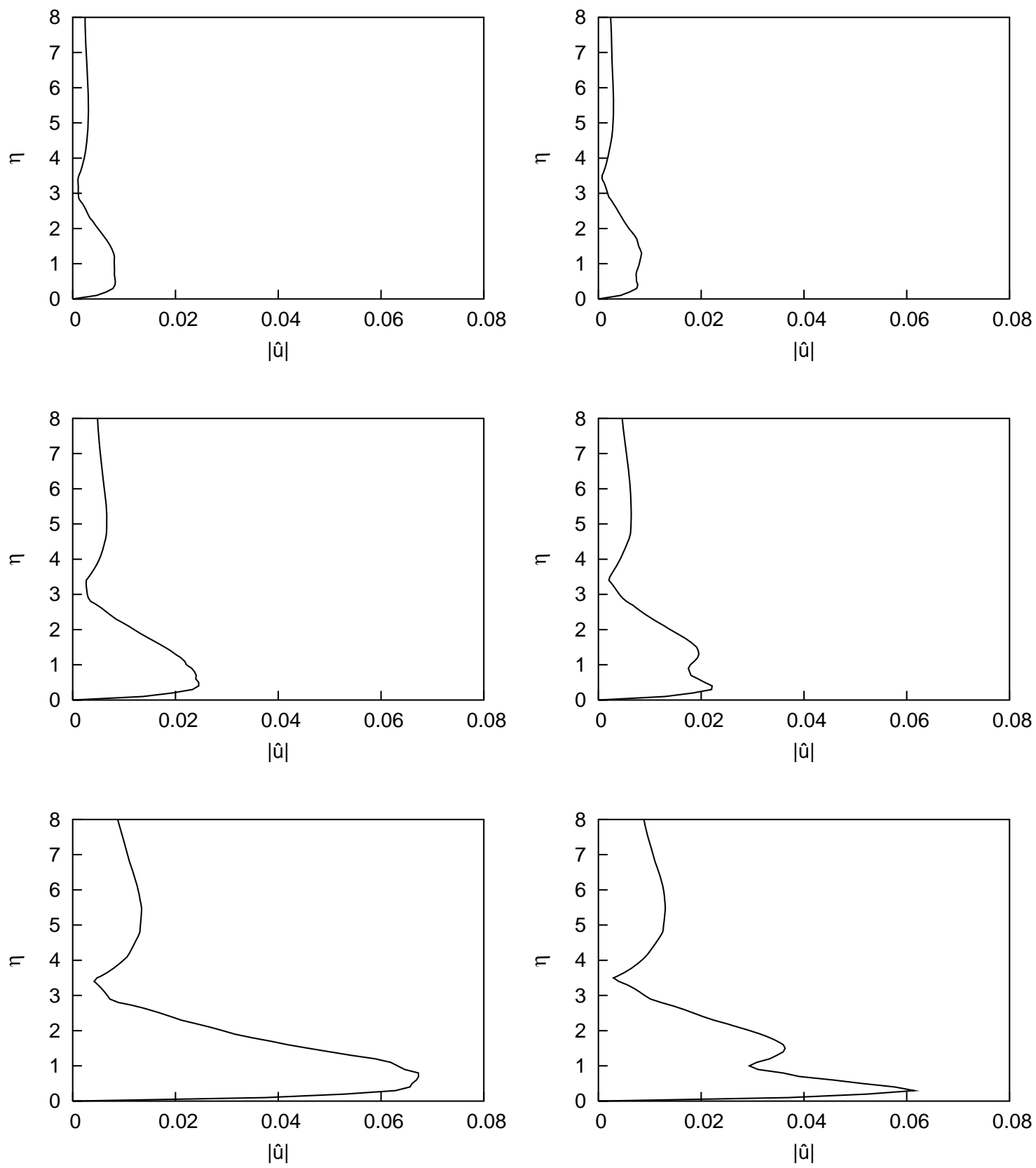

Figure 19: Conf. I - Evolution of the unsteady disturbance profiles in the high speed (left) and low speed streak (right) regions for $\mathrm{Re}_{\mathrm{k}}=153, \mathrm{R}=782,875$ and 946 (top to bottom) 

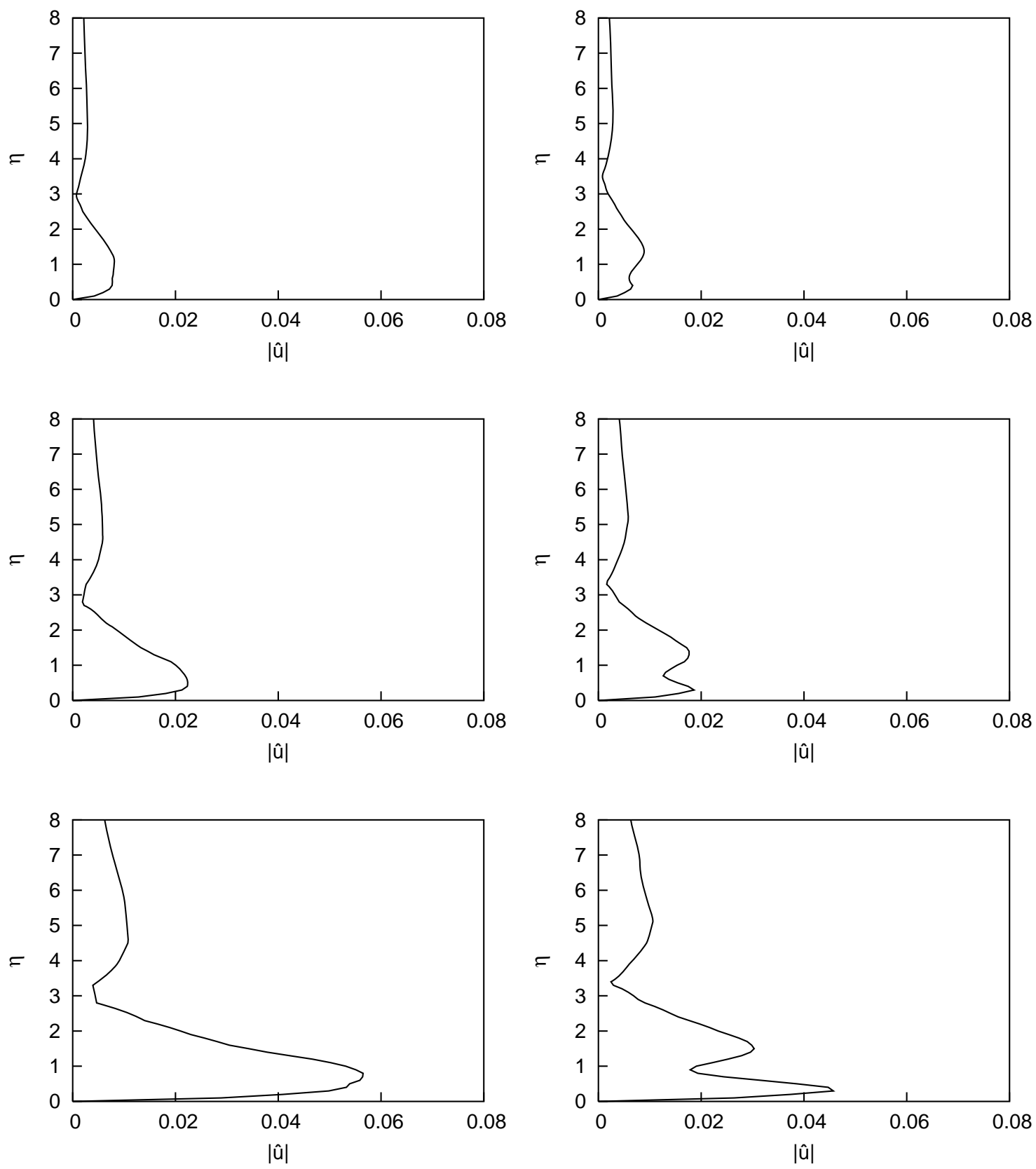

Figure 20: Conf. I - Evolution of the unsteady disturbance profiles in the high speed (left) and low speed streak (right) regions for $\mathrm{Re}_{\mathrm{k}}=238, \mathrm{R}=782,875$ and 946 (top to bottom) 

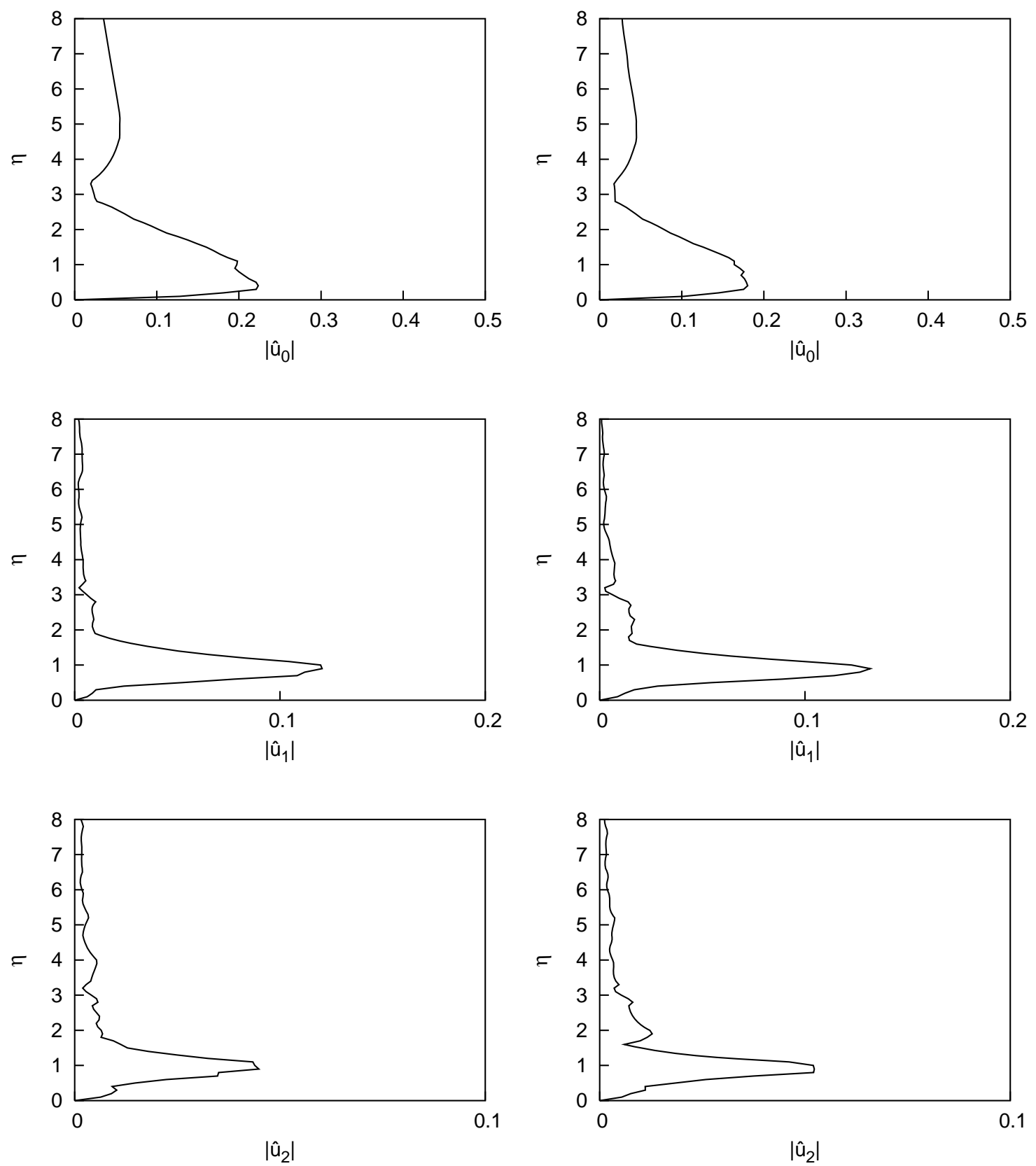

Figure 21: Conf. I - Spanwise modes of unsteady fluctuations for $\mathrm{Re}_{\mathrm{k}}=153$ (left) and $\mathrm{Re}_{\mathrm{k}}=238$ (right) at $\mathrm{R}=934$ 


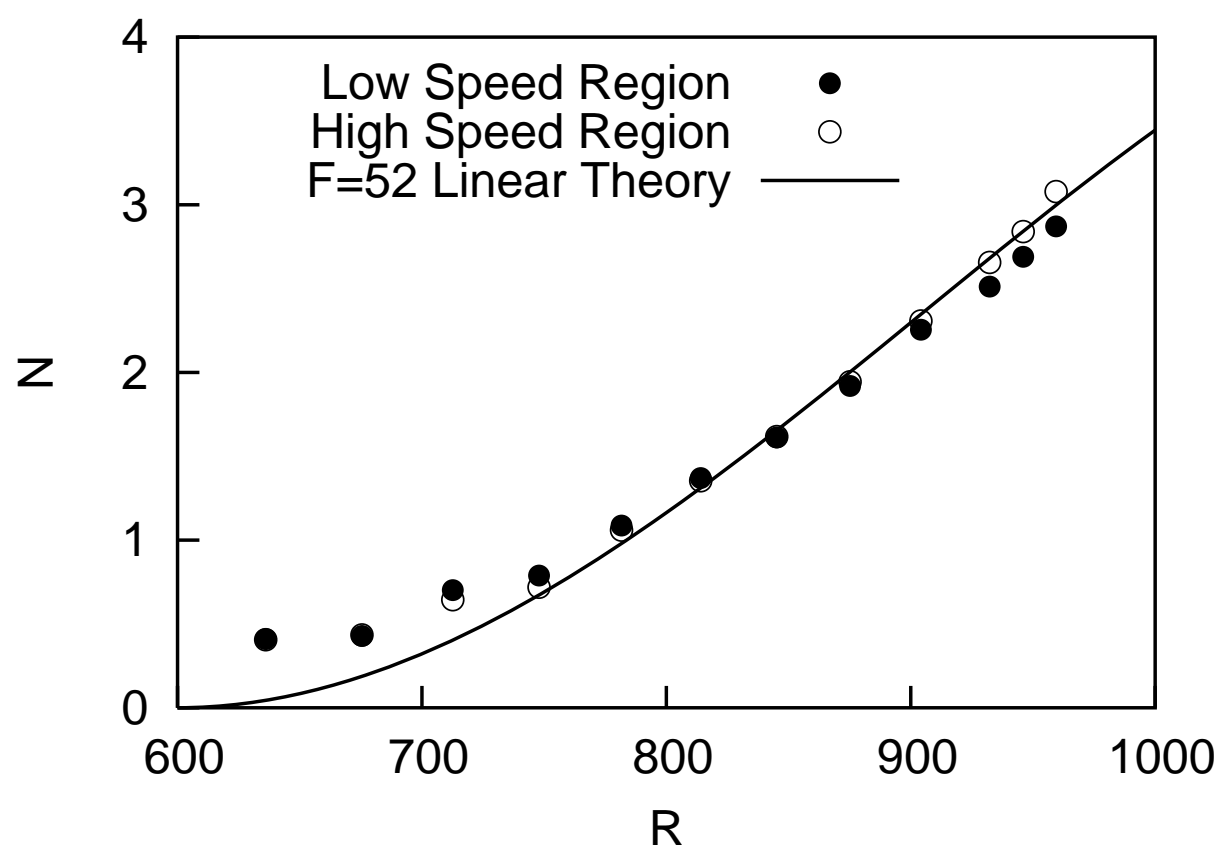

Figure 22: Conf. I - N-factor curves for $\mathrm{Re}_{\mathrm{k}}=153$

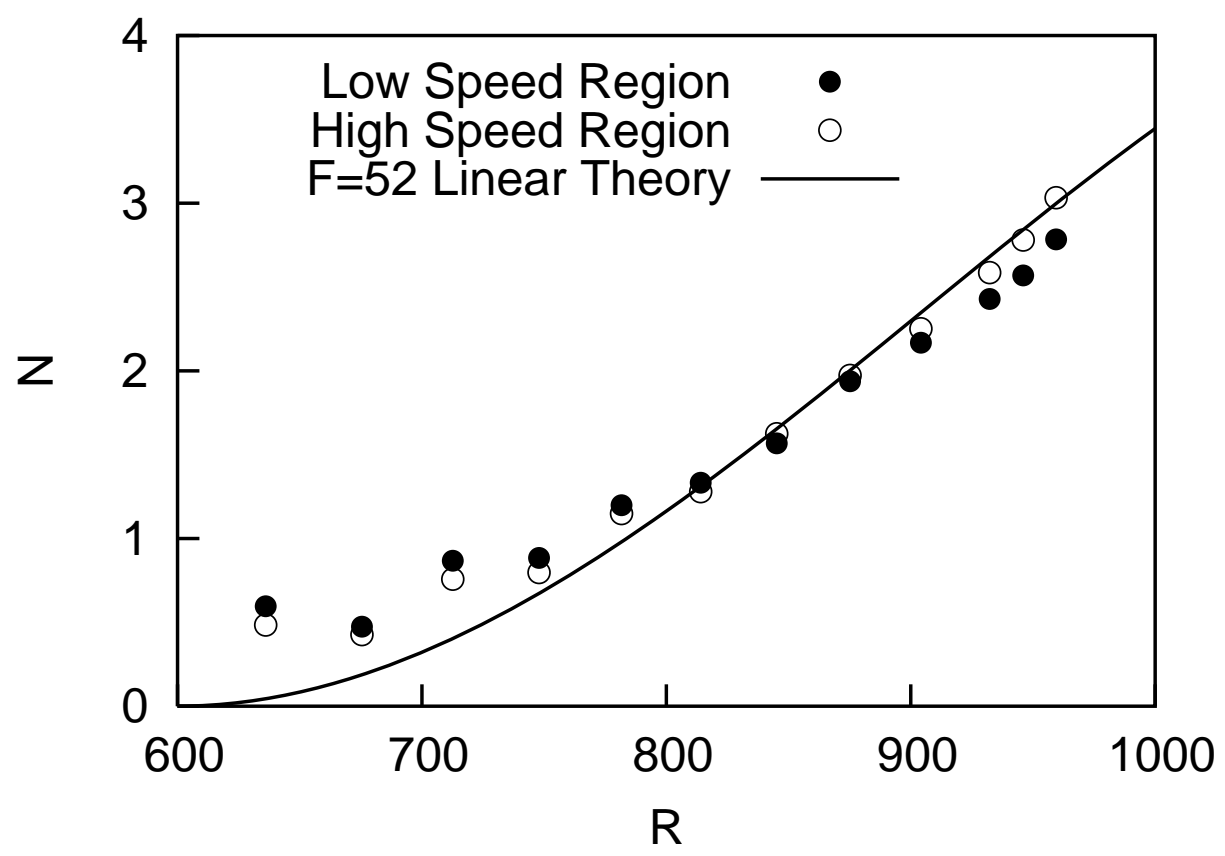

Figure 23: Conf. I - N-factor curves for $\mathrm{Re}_{\mathrm{k}}=238$ 

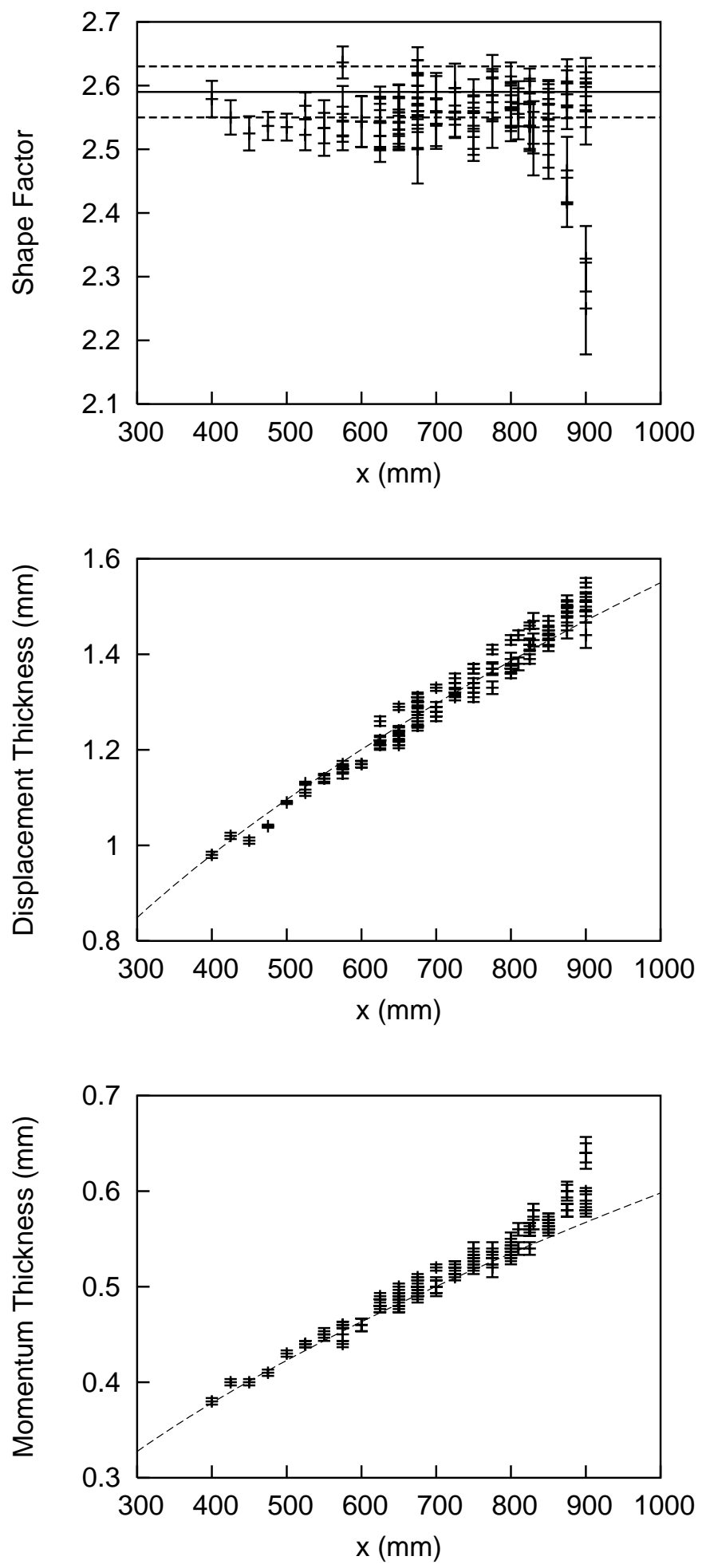

Figure 24: Conf. II - Displacement and Momentum Thicknesses, Shape Factor 


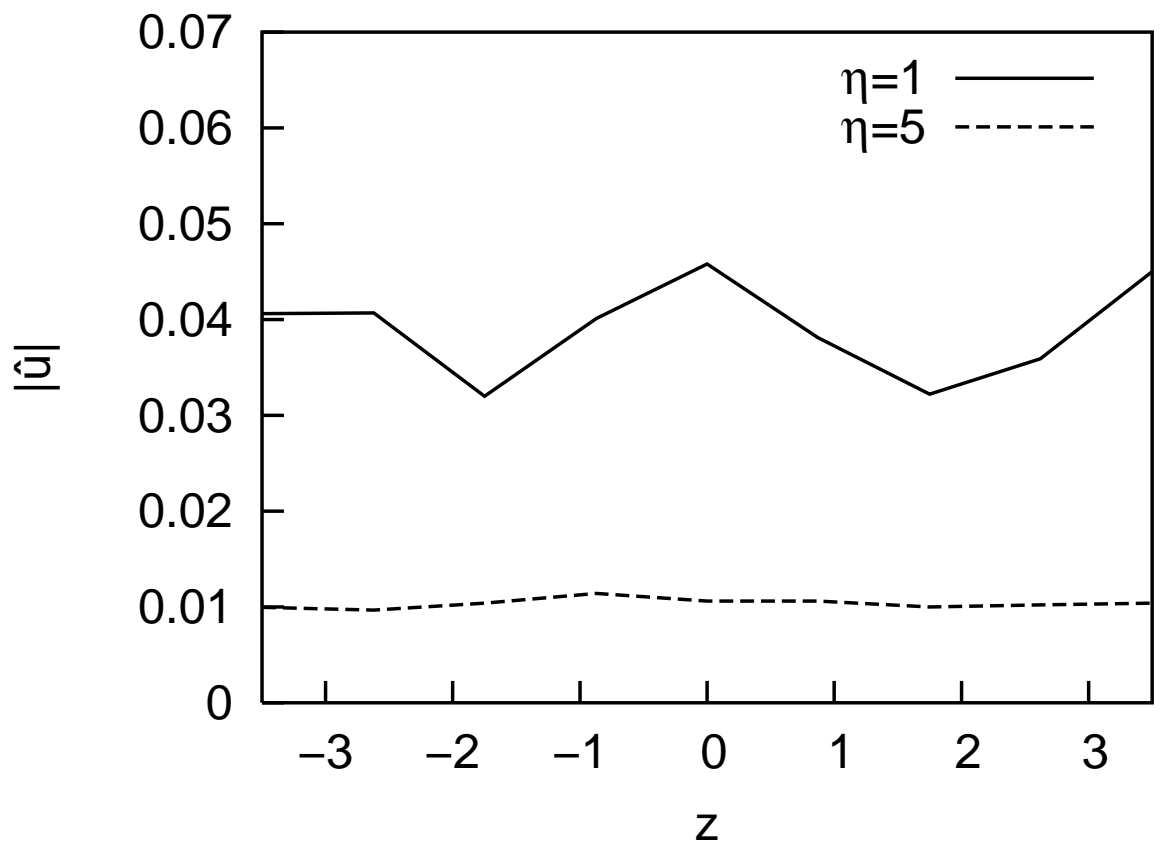

Figure 25: Spanwise variation of TS wave amplitudes with no basic state modulation, $\mathrm{R}=860$

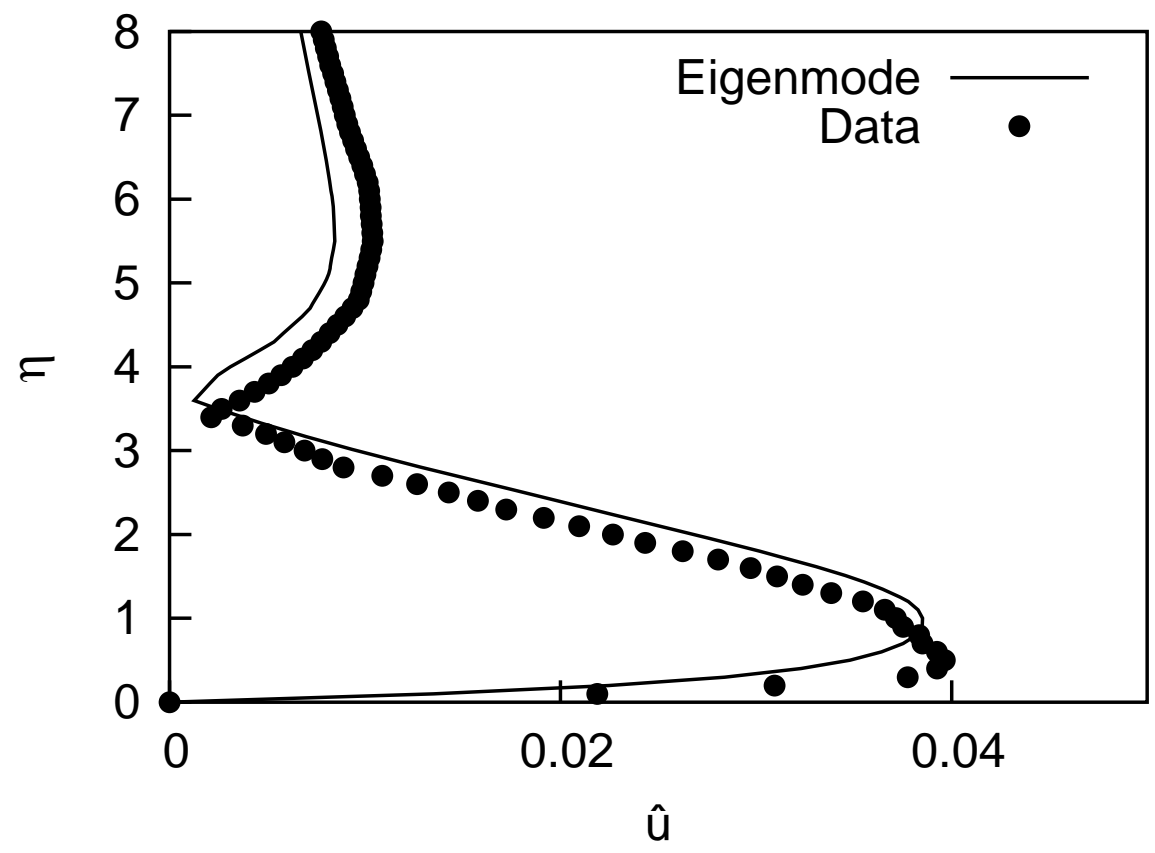

Figure 26: Conf. II - Spanwise mean TS wave profile at $\mathrm{R}=860$ 


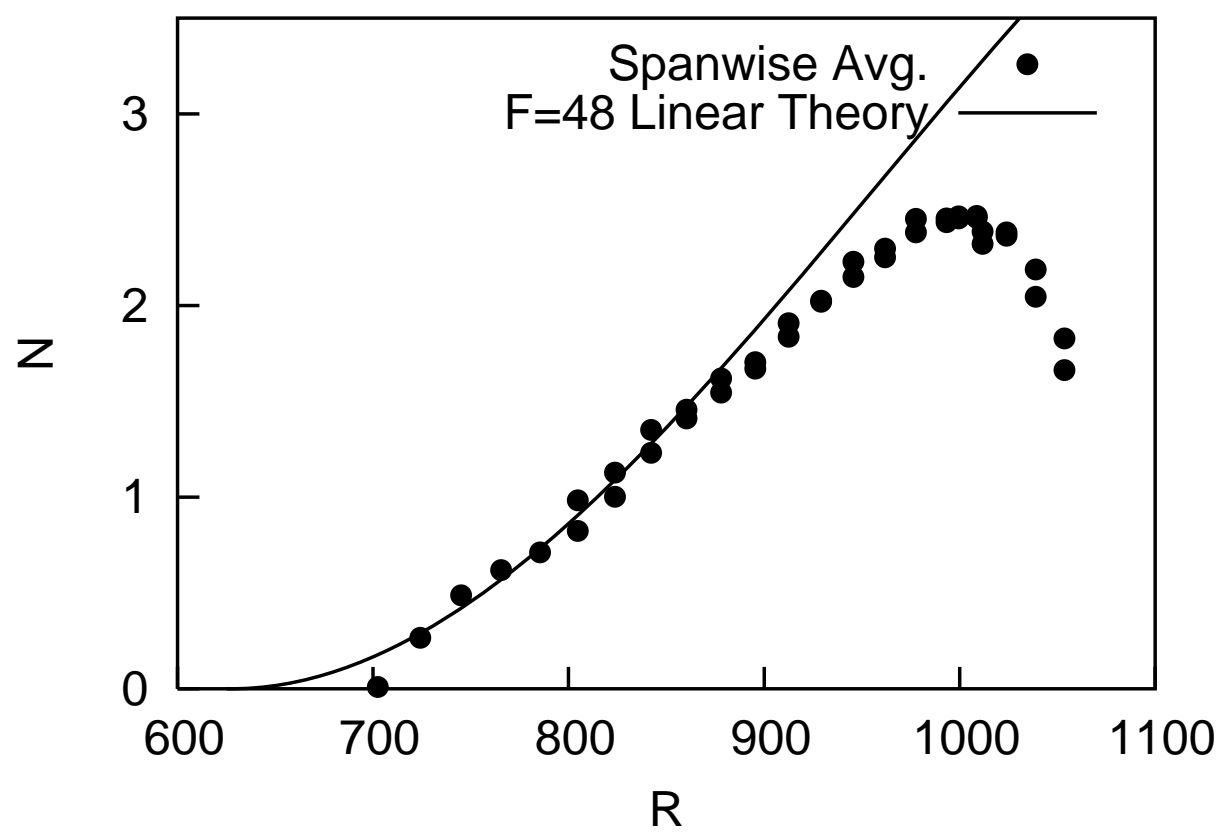

Figure 27: Spanwise averaged TS wave amplitude N-factors

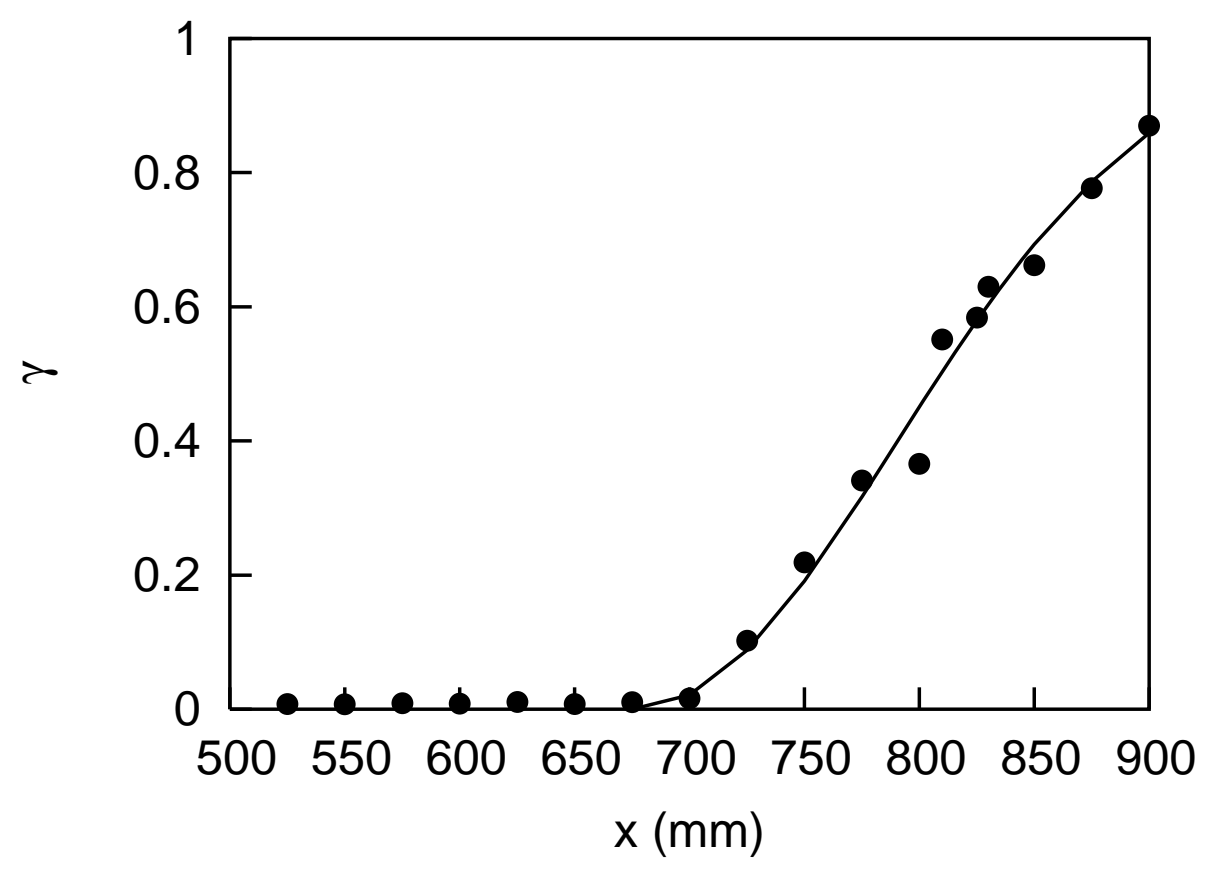

Figure 28: Streamwise development of intermittency with no basic state modulation 


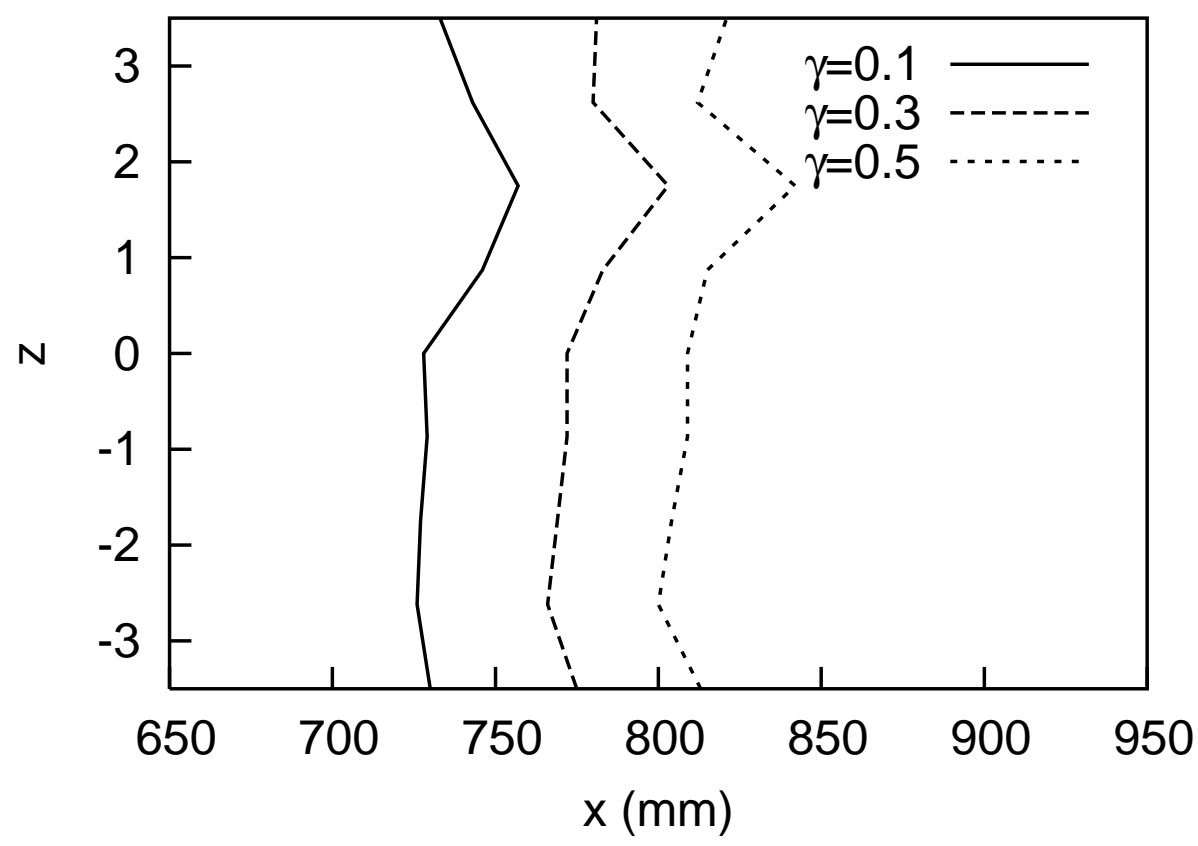

Figure 29: Intermittency contours with no basic state modulation 

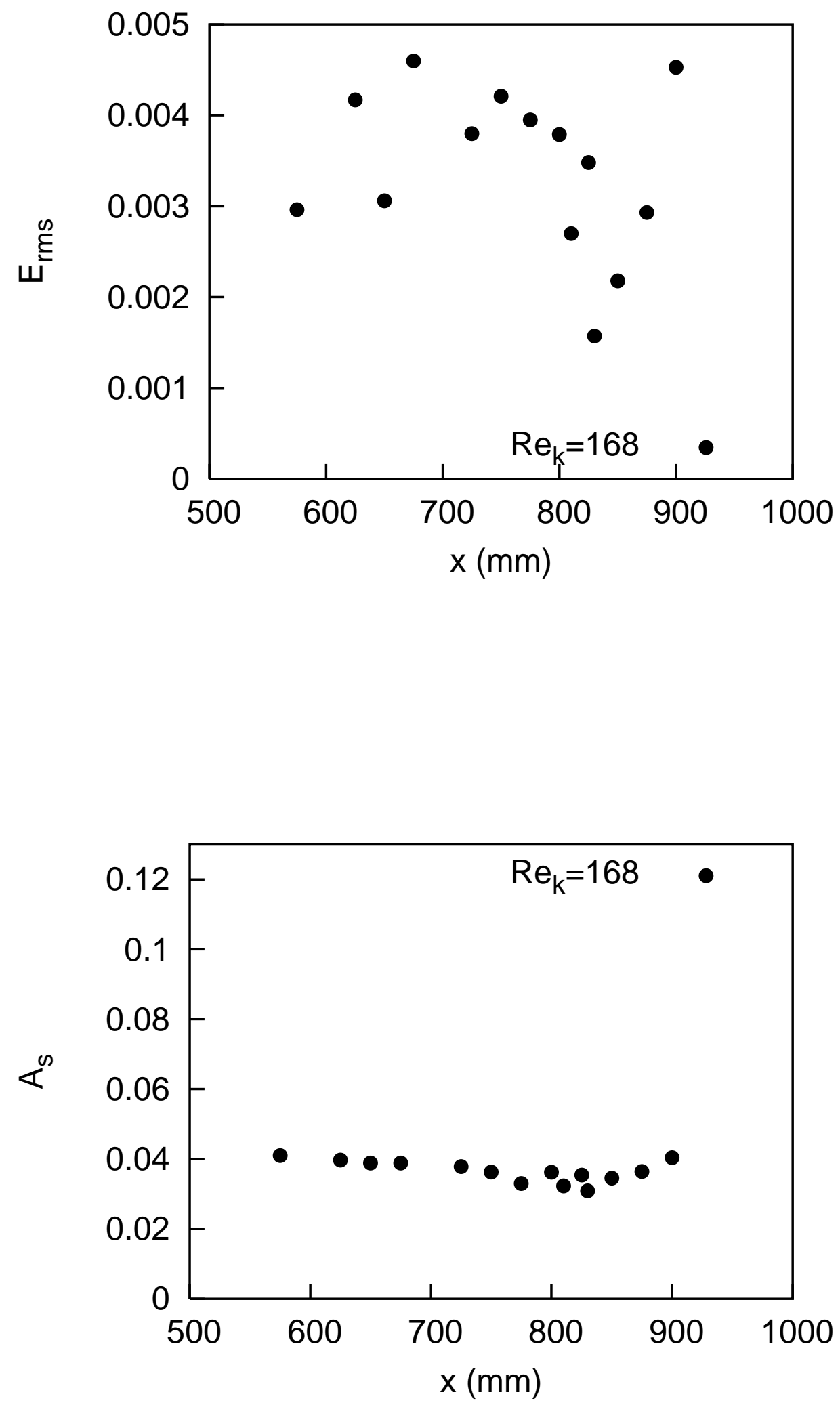

Figure 30: Conf. II - $\mathrm{E}_{\mathrm{rms}}$ and $\mathrm{A}_{\mathrm{s}}$ variation for $\mathrm{Re}_{\mathrm{k}}=168$ 

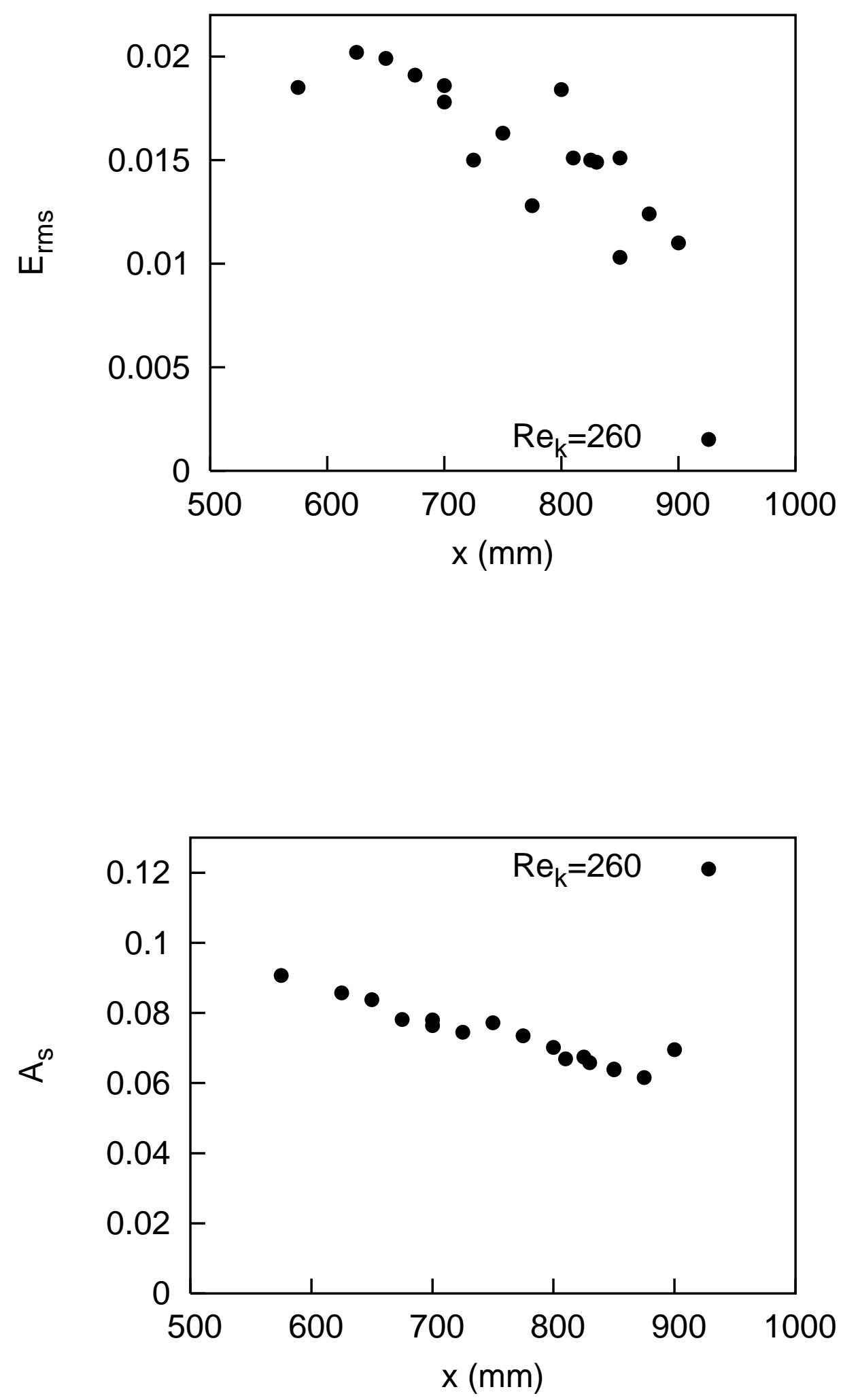

Figure 31: Conf. II - $\mathrm{E}_{\mathrm{rms}}$ and $\mathrm{A}_{\mathrm{s}}$ variation for $\mathrm{Re}_{\mathrm{k}}=260$ 


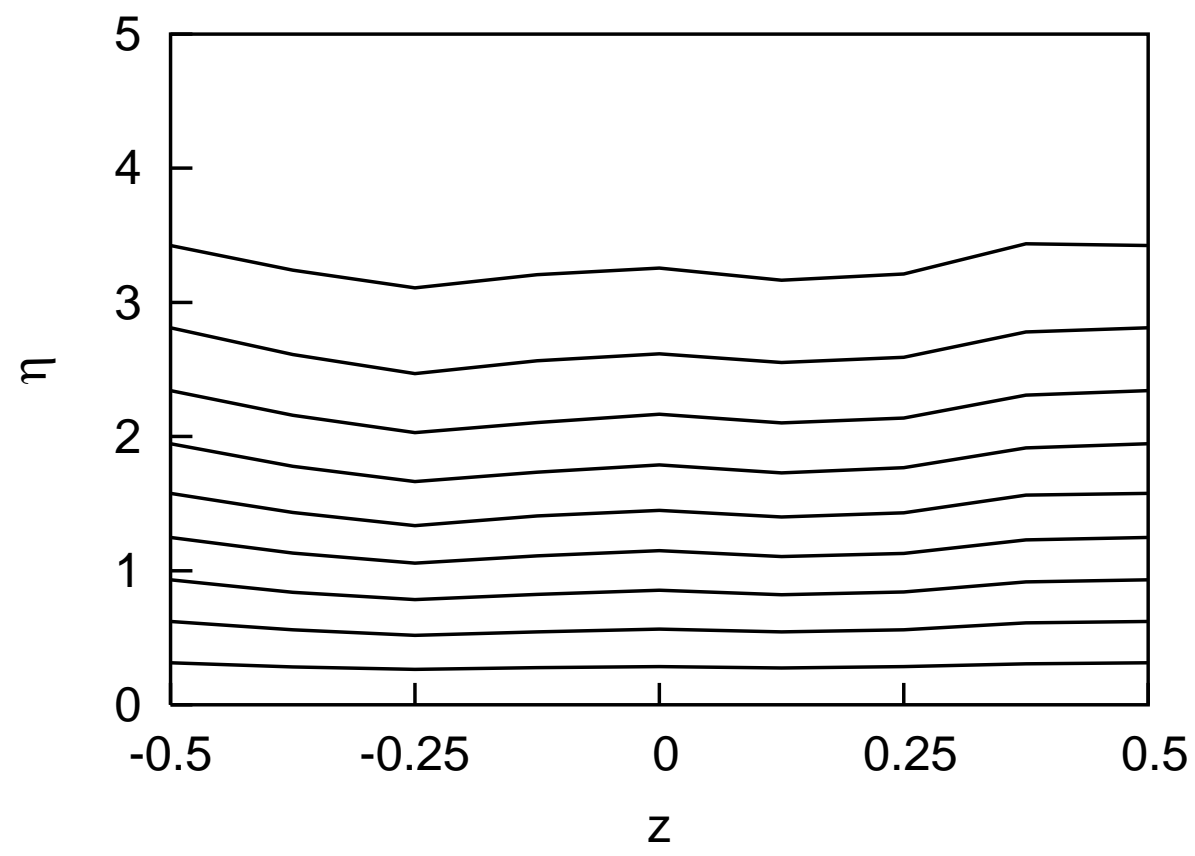

Figure 32: Conf. II - Velocity contours for $\mathrm{Re}_{\mathrm{k}}=168$ at $\mathrm{R}=842$

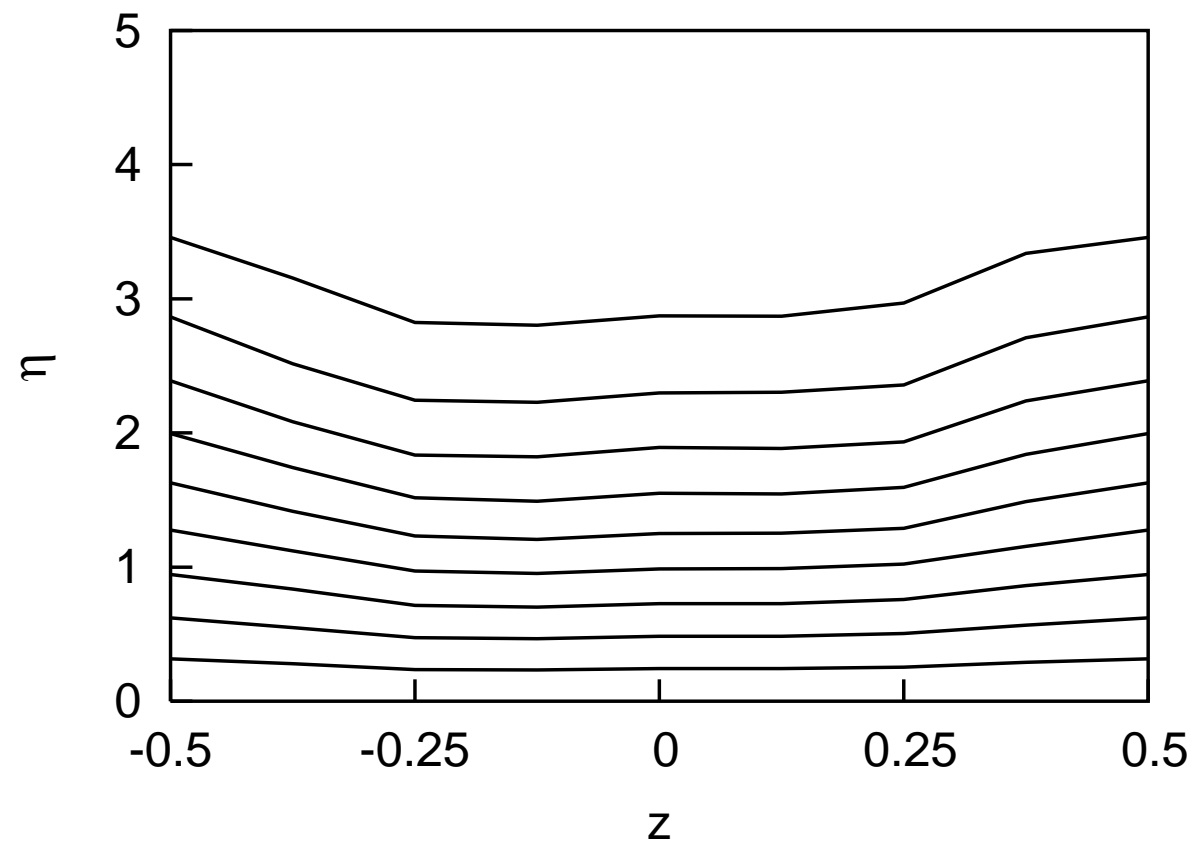

Figure 33: Conf. II - Velocity contours for $\mathrm{Re}_{\mathrm{k}}=260$ at $\mathrm{R}=946$ 

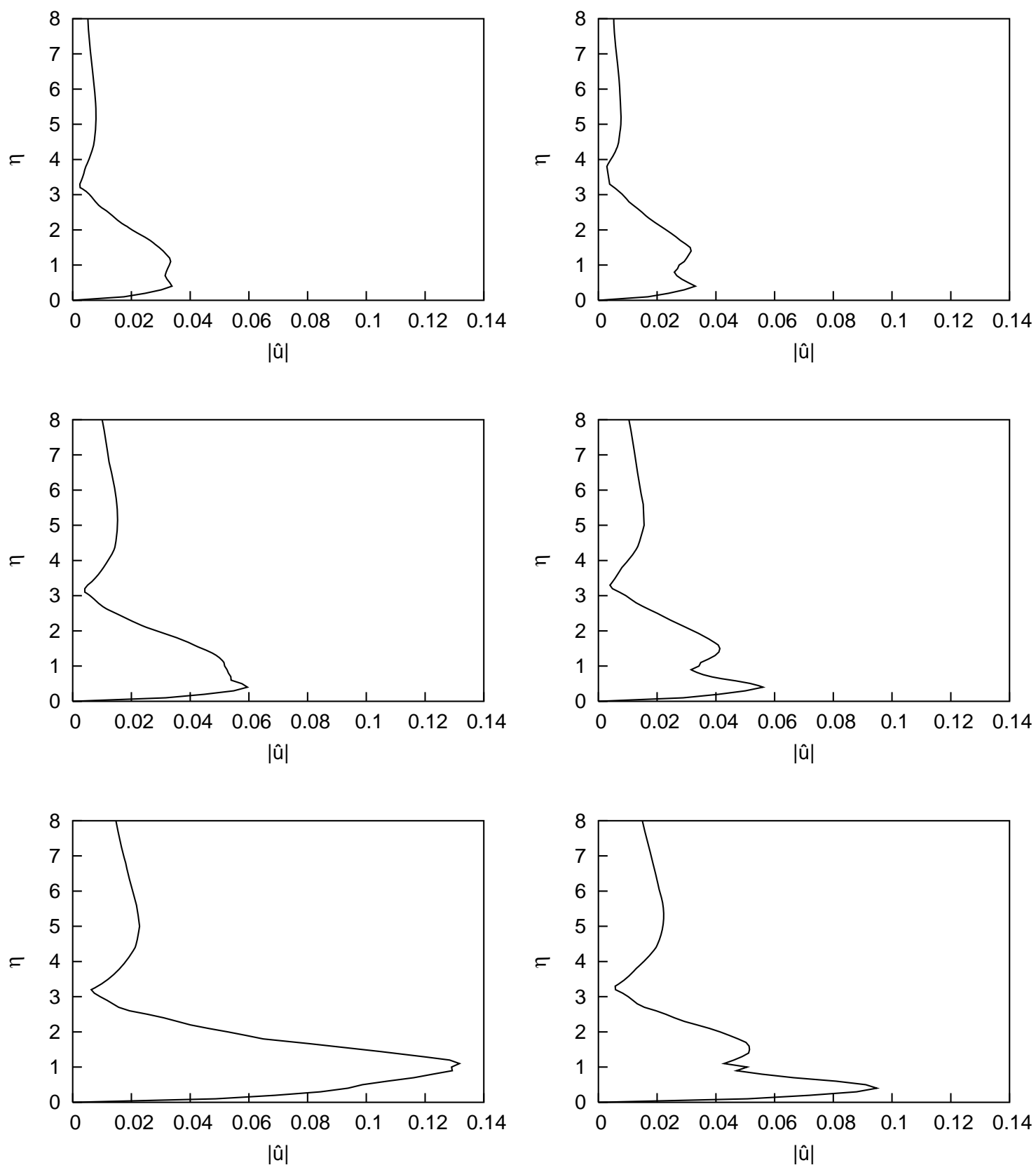

Figure 34: Conf. II - Evolution of the unsteady disturbance profiles in the high speed (left) and low speed streak (right) regions for $\mathrm{Re}_{\mathrm{k}}=168, \mathrm{R}=895,946$ and 1009 (top to bottom) 

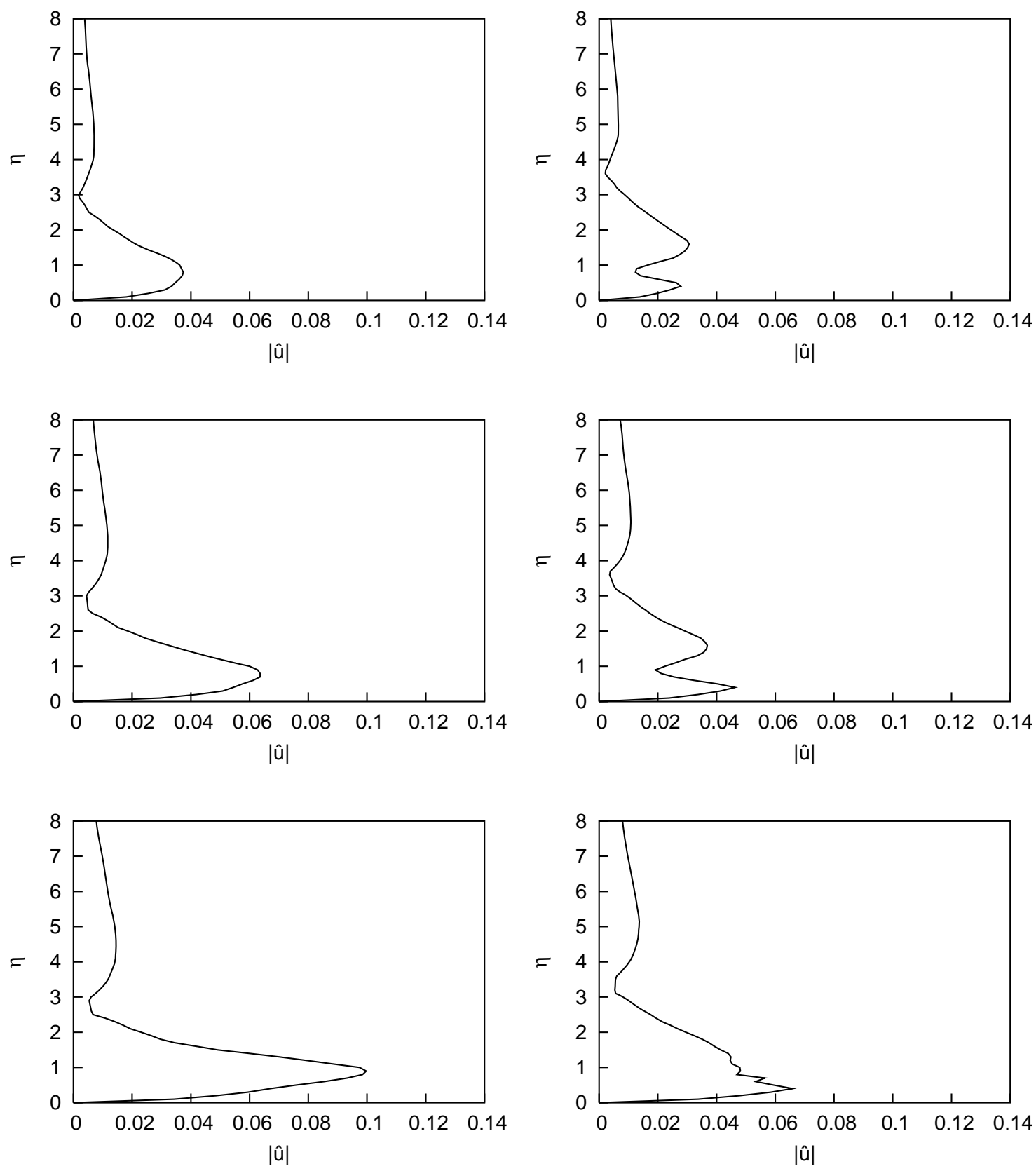

Figure 35: Conf. II - Evolution of the unsteady disturbance profiles in the high speed (left) and low speed streak (right) regions for $\mathrm{Re}_{\mathrm{k}}=260, \mathrm{R}=895,962$ and 1009 (top to bottom) 

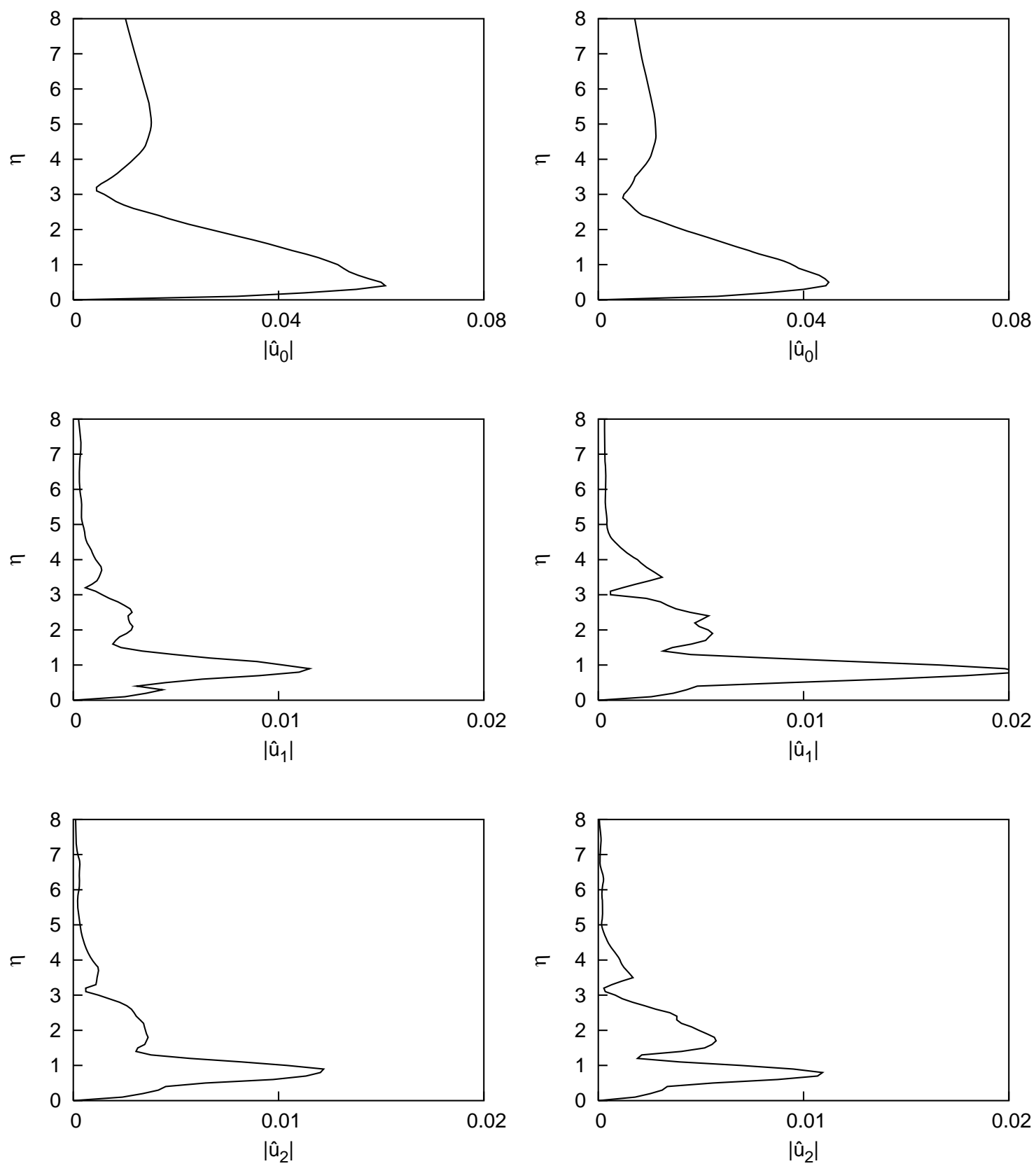

Figure 36: Conf. II - Spanwise modes of unsteady fluctuations for $\mathrm{Re}_{\mathrm{k}}=168$ (left) and $\mathrm{Re}_{\mathrm{k}}=260$ (right) at $\mathrm{R}=946$ 


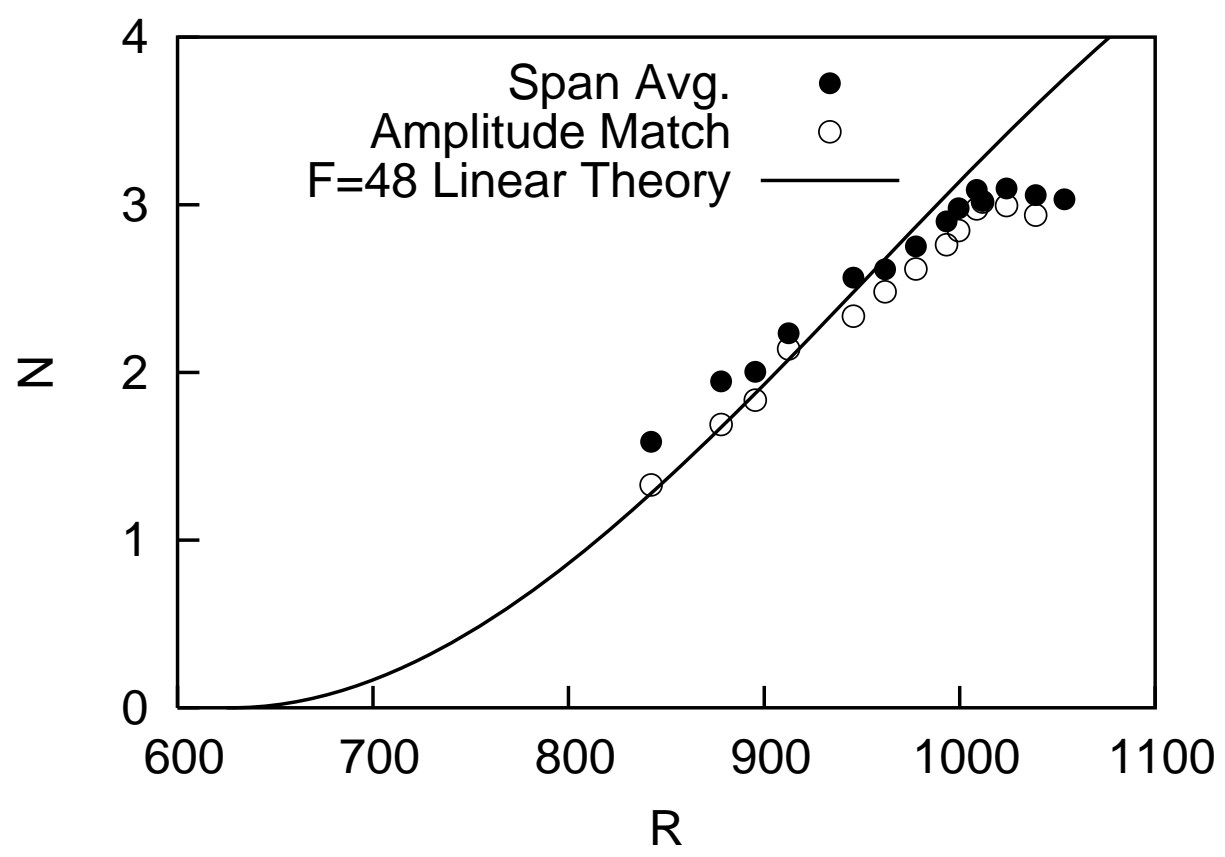

Figure 37: Conf. II - N-factor curves for $\mathrm{Re}_{\mathrm{k}}=168$

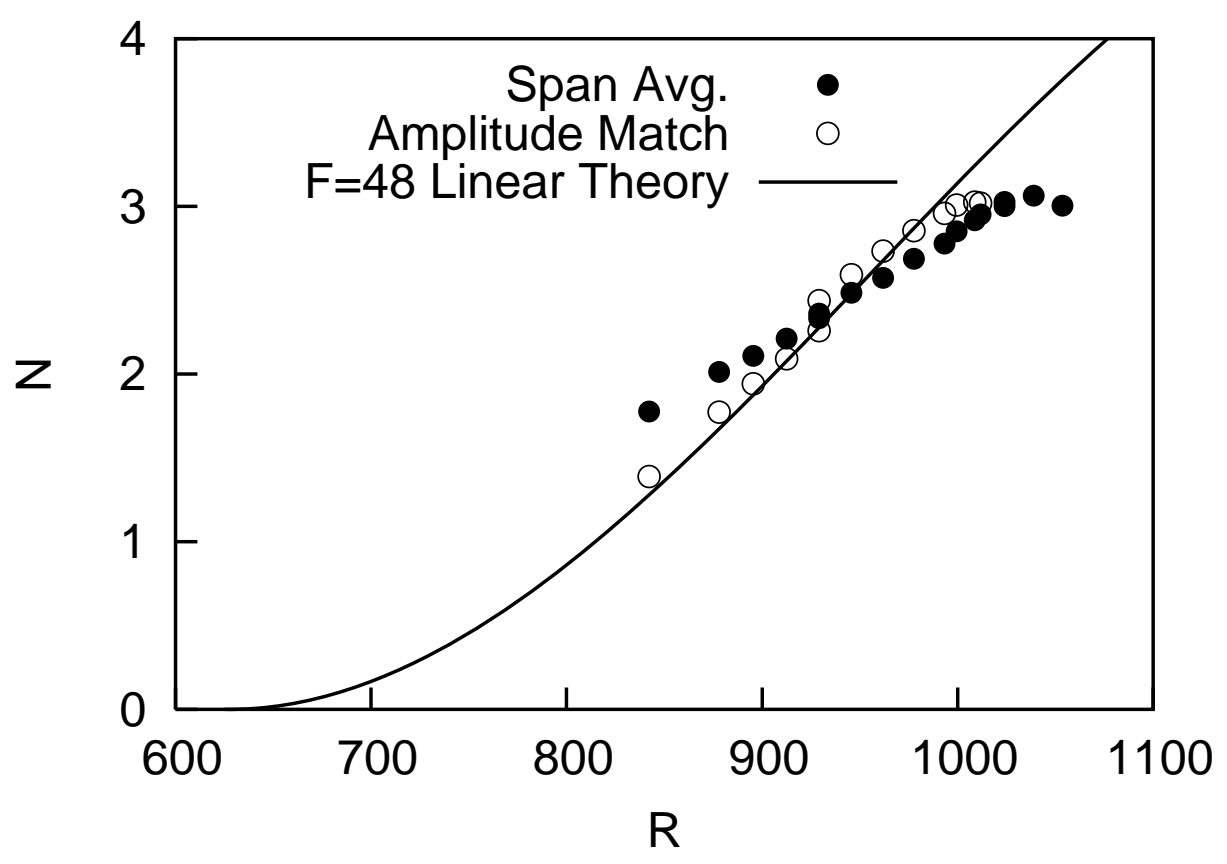

Figure 38: Conf. II - N-factor curves for $\mathrm{Re}_{\mathrm{k}}=260$ 


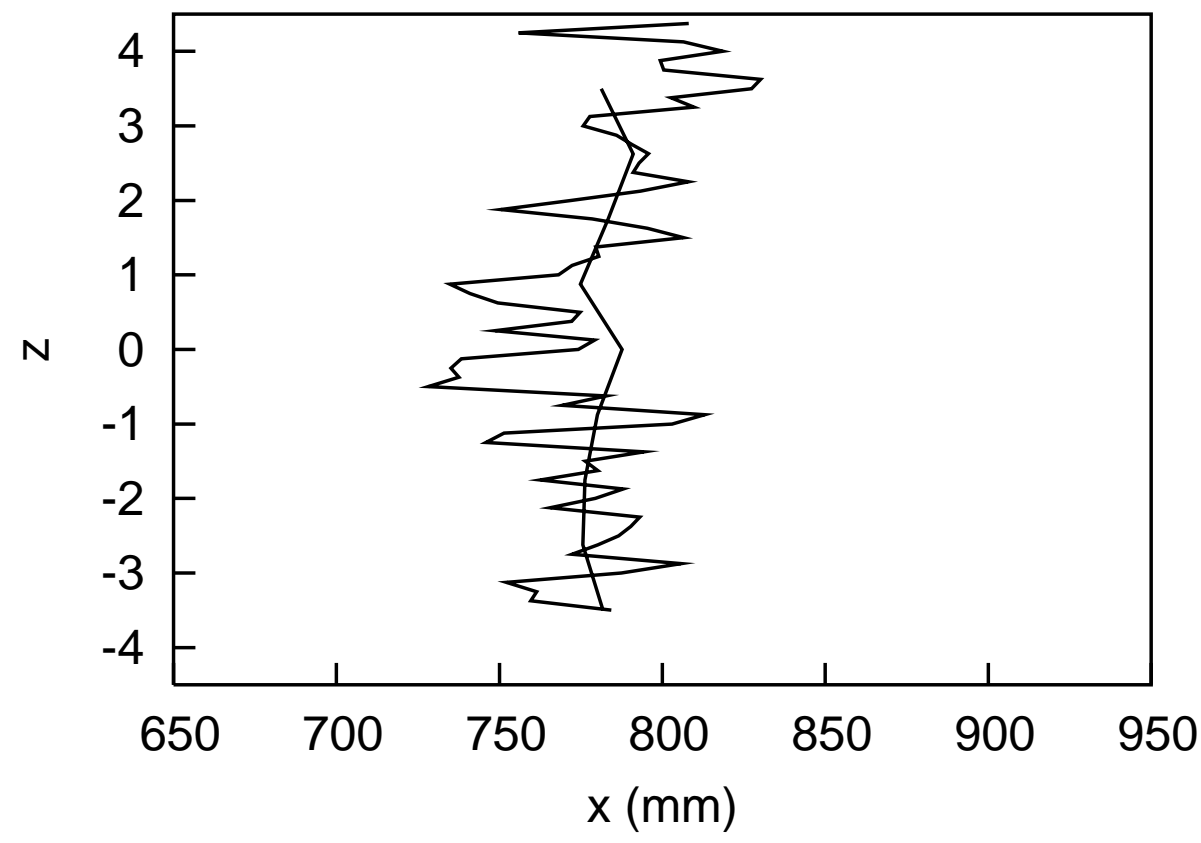

Figure 39: Contours of 10\% Intermittency with modulated basic state (thin line) and amplitude-matched TS waves for $\mathrm{Re}_{\mathrm{k}}=168$

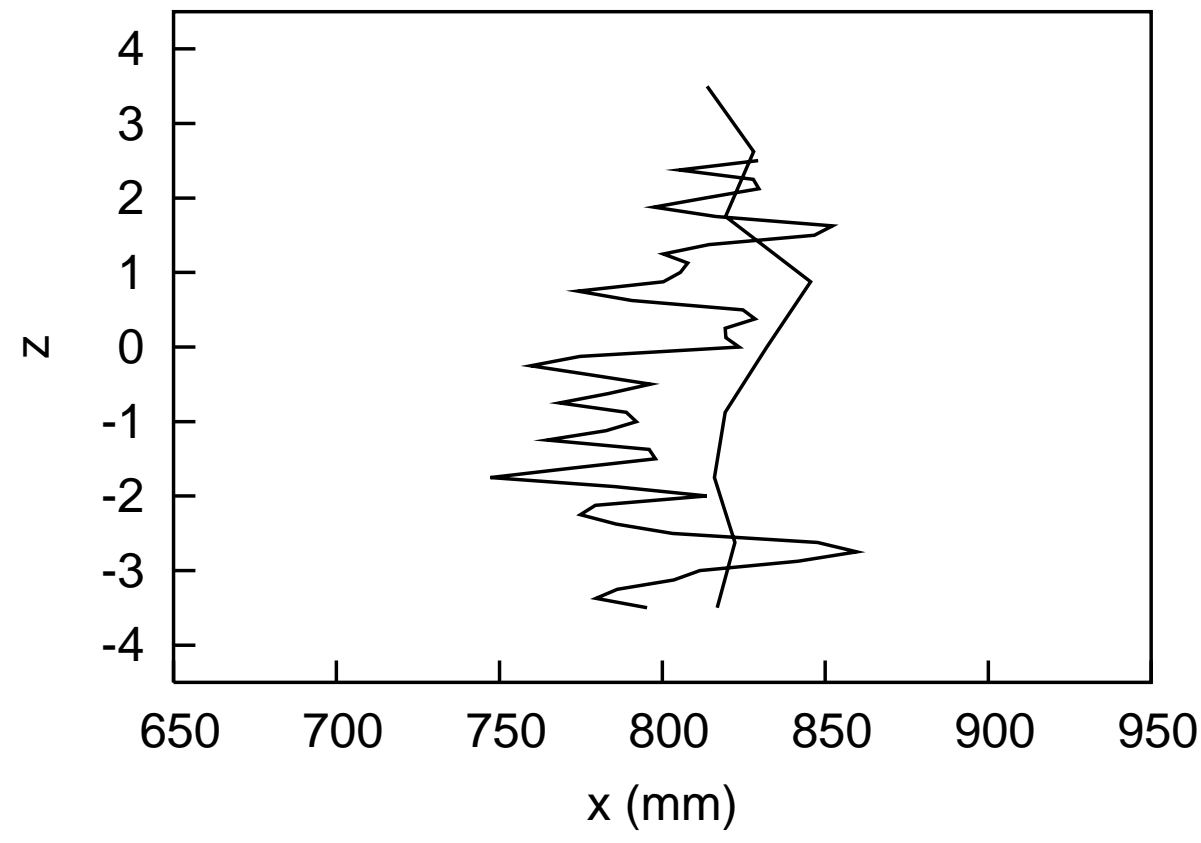

Figure 40: Contours of 10\% Intermittency with modulated basic state (thin line) and amplitude-matched TS waves for $\mathrm{Re}_{\mathrm{k}}=260$ 

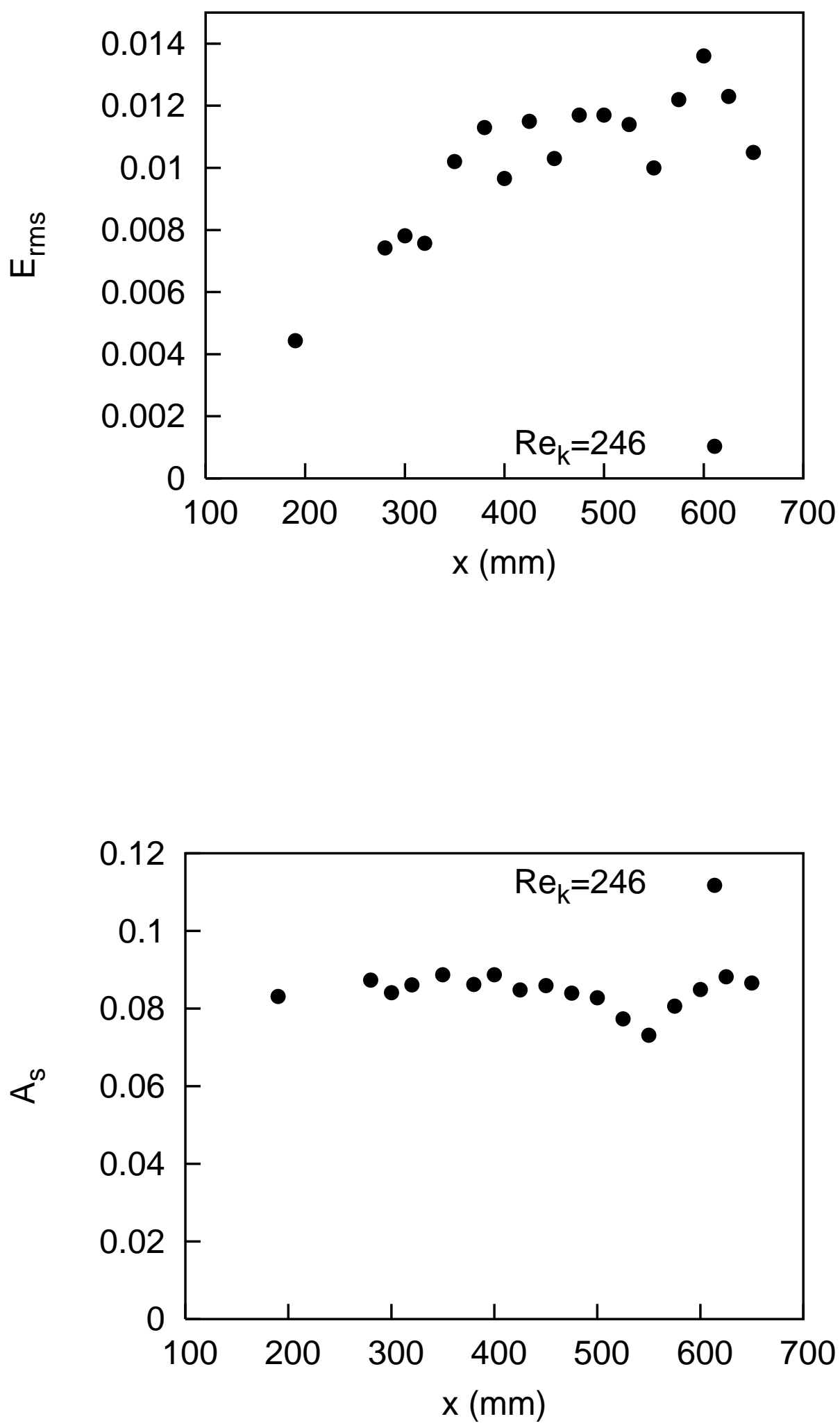

Figure 41: Conf. III - $\mathrm{E}_{\mathrm{rms}}$ and $\mathrm{A}_{\mathrm{s}}$ variation for $\mathrm{Re}_{\mathrm{k}}=246$ 

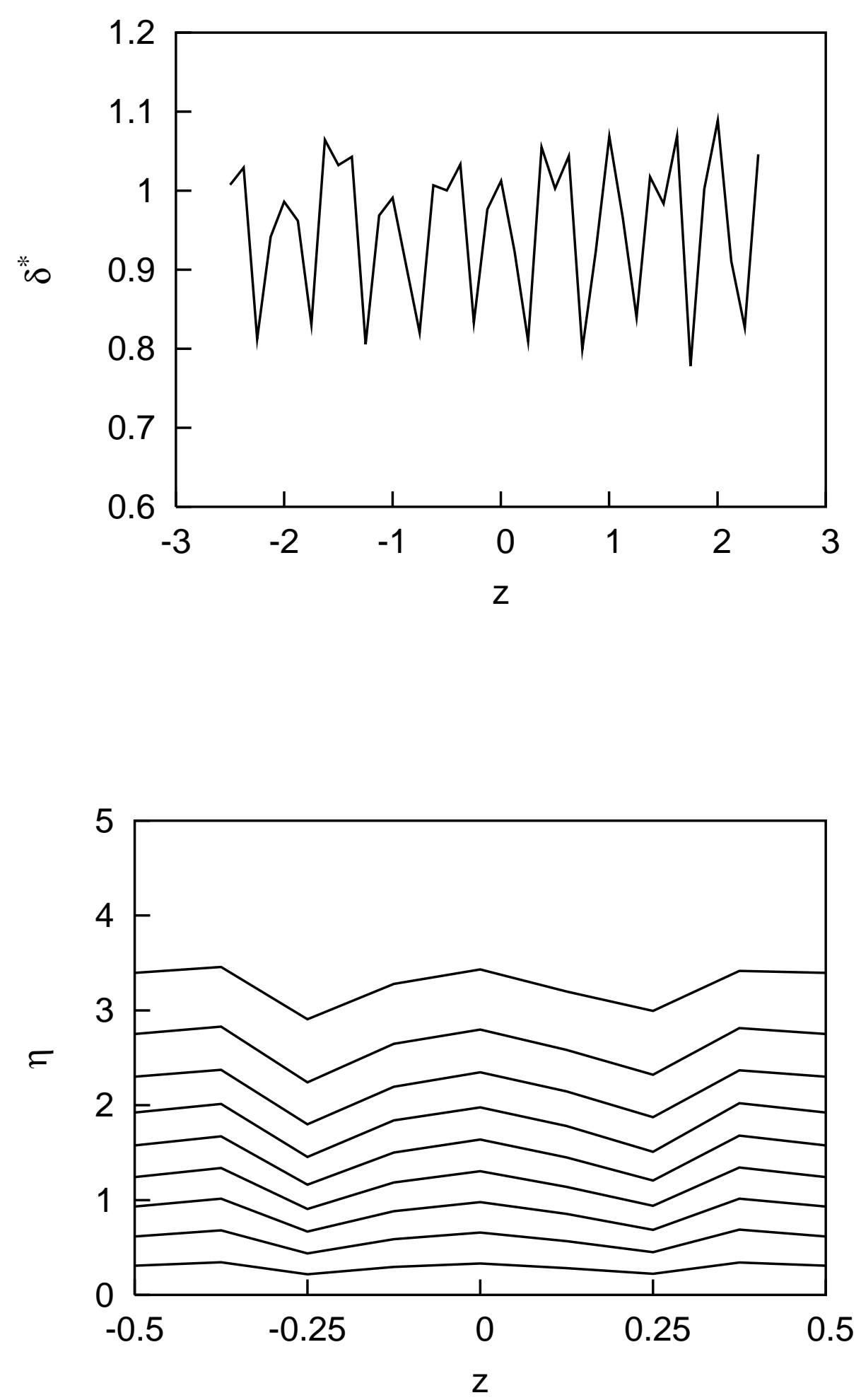

Figure 42: Conf. III - Displacement thickness variation and the velocity contours for $\mathrm{Re}_{\mathrm{k}}=246$ at $\mathrm{R}=574$ 

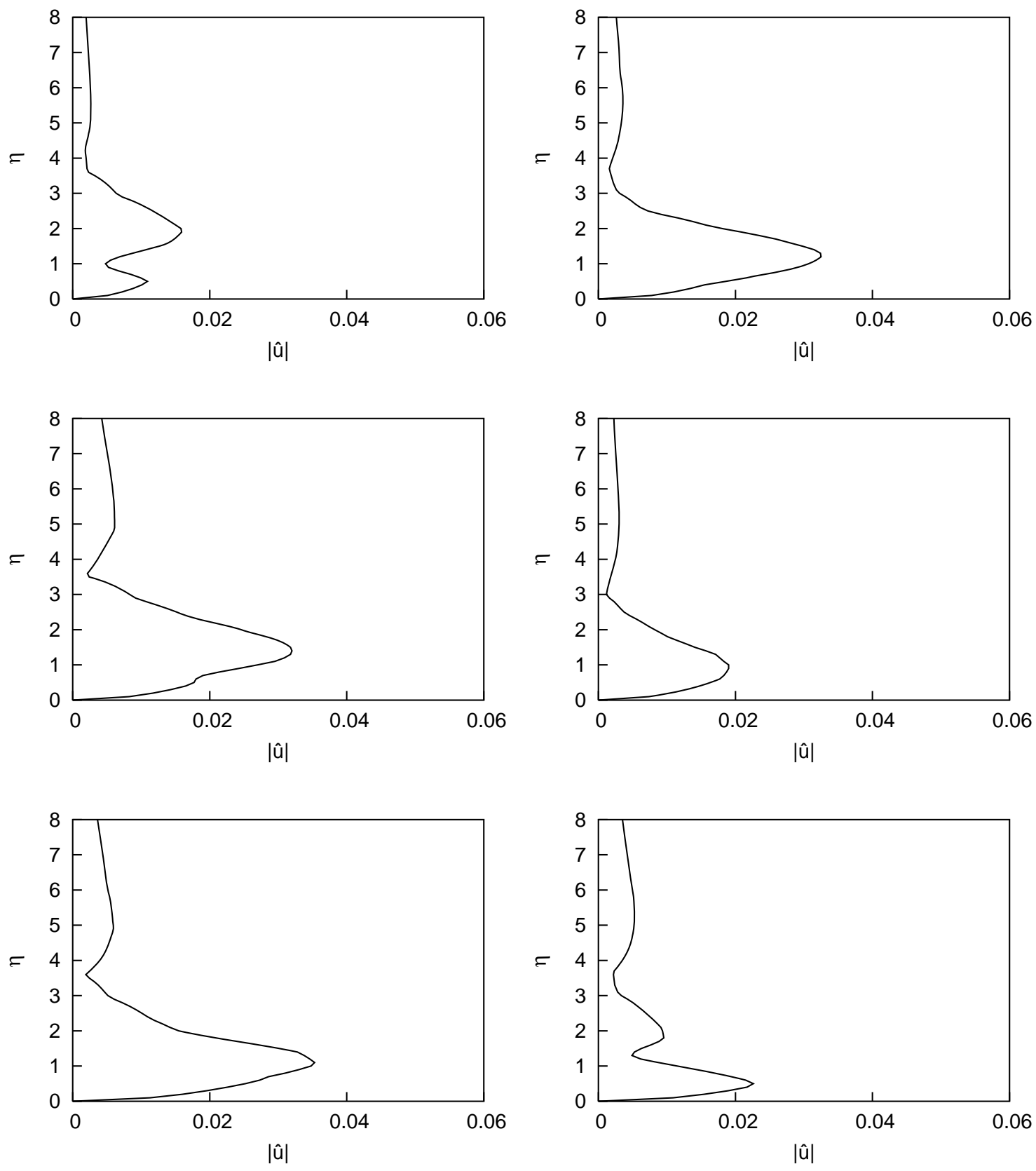

Figure 43: Conf. III - Evolution of the unsteady disturbance profiles in the high speed (left) and low speed streak (right) regions for $\mathrm{Re}_{\mathrm{k}}=246, \mathrm{R}=620,730$ and 759 (top to bottom) 

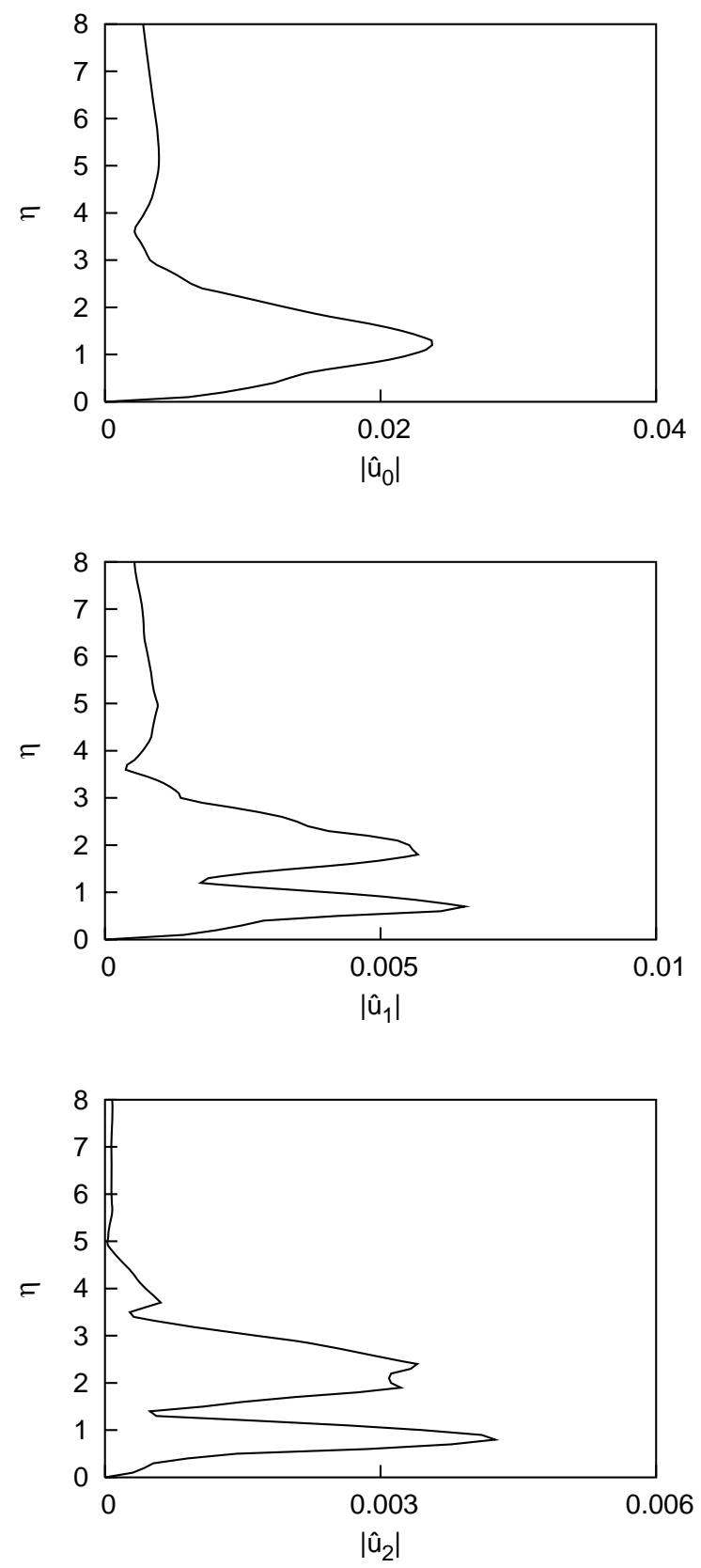

Figure 44: Conf. III- Spanwise modes of unsteady fluctuations for $\mathrm{Re}_{\mathrm{k}}=246$ at $\mathrm{R}=663$ 


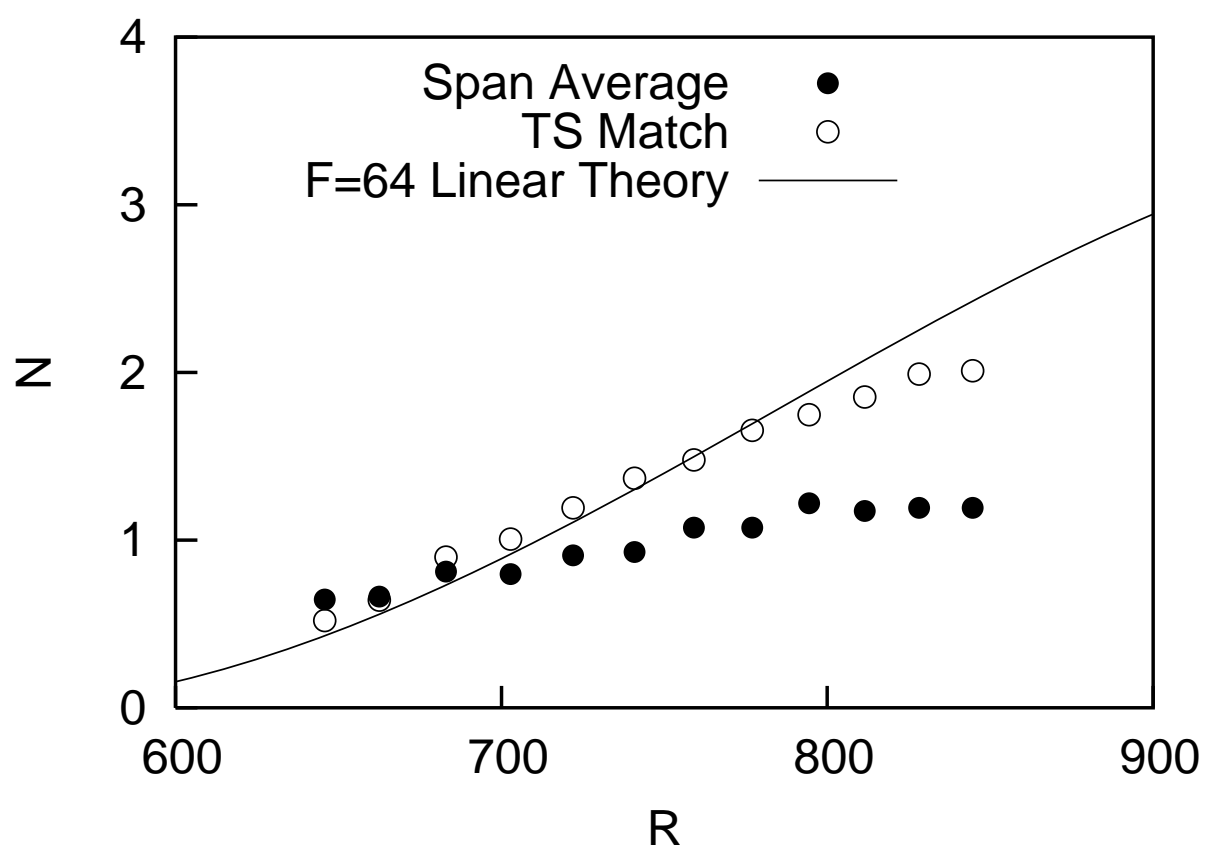

Figure 45: Conf. III - N-factor curves for $\mathrm{Re}_{\mathrm{k}}=246$

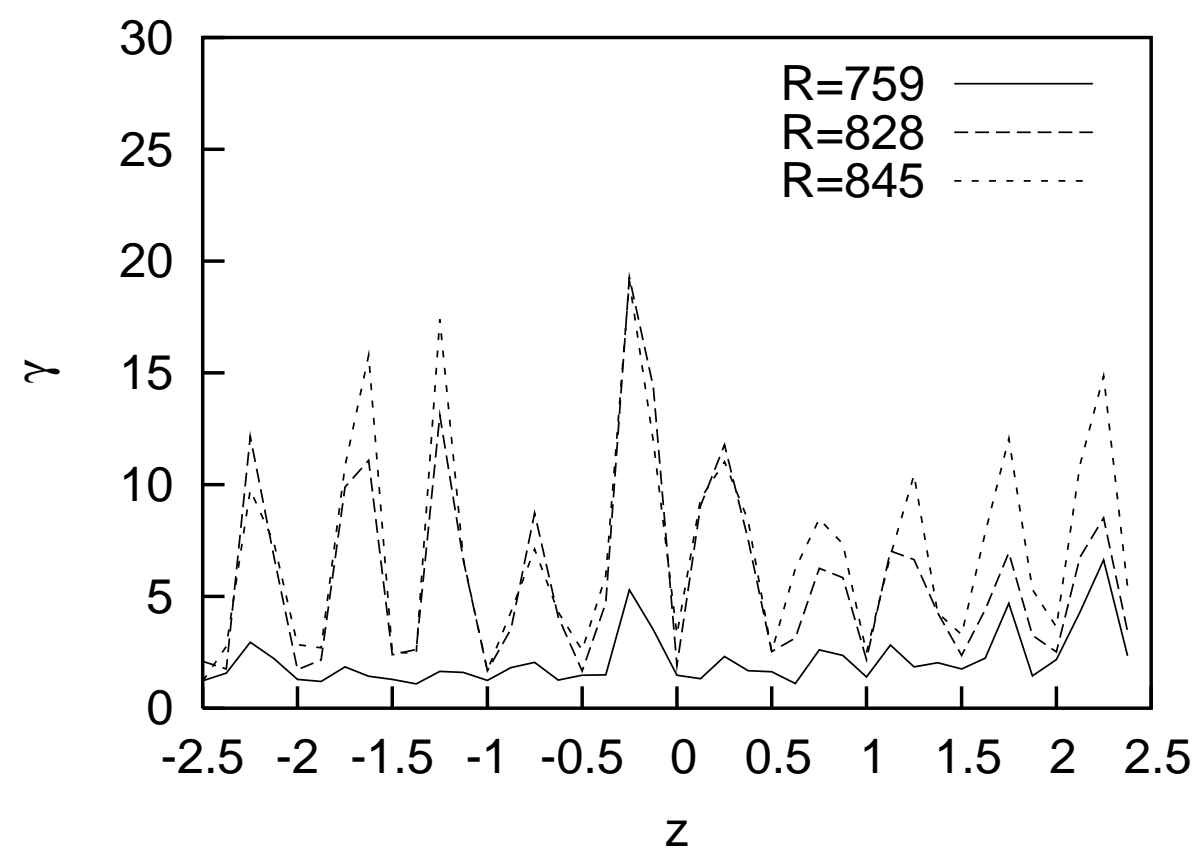

Figure 46: Conf. III - Intermittency variation across span for $\mathrm{Re}_{\mathrm{k}}=246$ 


\section{BIBLIOGRAPHY}

Andersson, P., M. Berggren and D. S. Henningson (1999). Optimal disturbances and bypass transition in boundary layers. Phys. Fluids 11(1), 134-150.

Andersson, P., L. Brandt, B. Allessandro and D. S. Henningson (2001). On the breakdown of boundary layer streaks. J. Fluid Mech. 428. 29-60.

Boiko, A., K. Westin, B. Klingmann, V. Kozlov and P. Alfredsson (1994). Experiments in a boundary layer subjected to free stream turbulence. Part II. The role of TSwaves in the transition process. J. Fluid. Mech. 281. 219-245.

Corke, T. C., A. Bar-Sever and M. V. Morkovin (1986). Experiments on transition enhancement by distributed roughness. Phys. Fluids. 29(10). 3199-3213.

Cossu, C. and L. Brandt (2002). Stabilization of Tollmien-Schclichting waves by finite amplitude optimal streaks in the Blasius boundary layer. Phys. Fluids. 14(8). L57L60.

Cossu, C. and L. Brandt (2004). On Tollmien-Schlichting-like waves in streaky boundary layers. European J. Mech. B/Fluids. 23(6). 815-833.

Ellingsen, T. and E. Palm (1975). Stability of linear flow. Phys. Fluids. 18, 487.

Ergin, F. G. and E. B. White (2005). Unsteady and transitional flows behind roughness elements. Submitted to AIAA Journal.

Farrell, B. F. (1988). Optimal excitation of perturbations in viscous shear flow. Phys. Fluids. 31, 2093. 
Fransson, J., L. Brandt, A. Talamelli and C. Cossu (2005). Experimental study of the stabilization of Tollmien-Schlichting waves by finite amplitude streaks. Phys. Fluids. 17. 2005.

Fransson, J., M. Matsubara and P. Alfredsson (2005). Transition induced by freestream turbulence. J. Fluid Mech. 527. 1.

Gurun, A. M. and E. B. White (2005). Tollmien-Schlichting wave suppression and transition delay using stationary transient disturbances. AIAA Paper 2005-5313.

Gurun, A. M. and E. B. White (2004). Tollmien-Schlichting wave suppression using stationary transient disturbances. Bull. Am. Phys. Soc. 49(9). 143.

Hedley, T. and J. Keffer (1974). Turbulent/non-turbulent decisions in an intermittent flow. J. Fluid Mech. 64. 625-644.

Herbert, Th. (1985). Three-dimensional phenomena in the transitional flat-plate boundary layer. AIAA Paper 85-0489.

Herbert, Th. (1988). Secondary instability of boundary layers. Annu. Rev. Fluid Mech. 20. 487-526.

Hultgren, L. S. and L. H. Gustavsson (1981). Algebraic growth of disturbances in a laminar boundary layer. Phys. Fluids. 24, 1000.

Kendall, J. M. (1998). Experiments on boundary layer receptivity to free-stream turbulence. AIAA Paper 98-0530.

Klebanoff, P. S., Schubauer, G. and K. Tidstrom (1955). Measurements of the effect of the two-dimensional and three-dimensional roughness elements on boundary layer transition. J. Aero. Sci. 22. 803-804 
Klebanoff, P. S., K. D. Tidstrom and L. M. Sargent (1962). The three-dimensional nature of boundary layer instability. J. Fluid Mech. 12. 1-24.

Klebanoff, P. S. (1971). Effect of free-stream turbulence on the laminar boundary layer. Bull. Am. Phys. Soc. 10, 1323.

Kuan, C. and T. Wang (1990). Investigation of the intermittent behavior of transition boundary layer using a conditional averaging technique. Exp. Ther. Fluid Sci. 3. 157-173.

Landahl, M. T. (1980). A note on an algebraic instability of inviscid parallel shear flows. J. Fluid Mech. 98, 243-251.

Luchini, P. (2000). Reynolds-number-independent instability of the boundary layer over a flat surface: Optimal perturbations. J. Fluid Mech. 404, 289-309.

Morkovin, M.V. (1969). Viscous Drag Reduction. 1-31. Plenum Press., New York.

Morkovin, M. V., E. Reshotko and Th. Herbert (1994). Transition in open flow systemsA reassement. Bull. APS. 39(9), 1882.

Narasimha, R. (1957) On the distribution of intermittency in the transition region of a boundary layer. J. Aero. Sci. 24. 711-727.

Orr, W. M'F. (1907). The stability or instability of the steady motions of a perfect liquid and of a viscous liquid. Proc. Roy. Irish Acad. Sect. A. 27, 9-68, 69-138 .

Prandtl, L. (1921). Bemerkund uber die Entstehung der Turbulenz. Z. Angew. Math. Mech. 1, 136-139.

Rice, J. M. (2004). Roughness receptivity and scaling of non-optimal transient disturbances. Master’s Thesis. Case Western Reserve University. 
Saric W. S. (1990). Low-speed experiments: Requirements for stability measurements. Instability and Transition, I. Springer-Verlag, 174

Saric W. S. (1994). Physical description of boundary layer transition: Experimental evidence. AGARD Report No. 793.

Saric, W. S. and A. S. W. Thomas (1984). Experiments on the subharmonic route to turbulence in boundary layers. Turbulence and Chaotic Phenomena in Fluids. 117122. Elsevier, New York.

Saric, W. S., H. L. Reed and E. J. Kerschen (2002). Boundary-layer receptivity to freestream disturbances. Ann. Rev. Fluid. Mech. 34, 291-319.

Schlichting, H. (1933). Laminare Strahlenausbreitung. Z.Angew Math. Mech. 13, 260263.

Schubauer, G.B. and H. Skramstad (1947). Laminar boundary layer oscillations and transition on a flat plate. J. Res. Nat. Bur. Standards. 38, 251-292 .

Singer, B. A., H. L. Reed and J. H. Ferziger (1989). Effect of streamwise vortices on transition in plane channel flow. Phys. Fluids A. 1. 1960.

Sommerfeld, A. (1908). Ein Beitrag zur hydrodynamischen Erklaerung der turbulenten Fluessigkeitsbewegungen. Proc. Fourth Int. Cong. Math. III, 116-124.

Squire, H.B. (1933). On the stability of three-dimensional distribution of viscous fluid between parallel walls. Proc. Roy. Soc. ser A. 142, 541-546.

Tani, I. (1969). Boundary layer transition. Annu. Rev. Fluid. Mech. 1. 169-196

Thomas, A.S.W. (1985). Aircraft drag reduction technology. AGARD Report No. 723.

Tollmien, W. (1929). Uber die Entstehung der Turbulenz. Nachr. Ges. Wiss. Gottingen Math-Pyhs. Kl. II, 21-44. 
Tumin, A. and E. Reshotko. (2001). Spatial theory of optimal disturbances in boundary layers. Phys. Fluids 13(7), 2097-2104.

Westin, K. J. A., A. V. Boiko, B. G. B. Klingmann, V. V. Kozlov and P. H. Alfredsson (1994). Experiments in a boundary layer subjected to free stream turbulence. Part I. Boundary layer structure and receptivity. J. Fluid. Mech. 281, 193-218.

White, E. B. (2002). Transient growth of stationary disturbances in a flat plate boundary layer. Phys. Fluids. 14(12). 4429-4439.

White, E. B. and E. Reshotko (2002). Roughness-induced transient growth in a flat plate boundary layer. AIAA Paper 2002-0138.

White, E. B., M. A. Shelley and A. M. Gurun (2004). Interactions of TollmienSchlichting waves and stationary transient disturbances. AIAA Paper 2004-2540.

White, F. M. (1991). Viscous Fluid Flow. McGraw-Hill. New York. 